\title{
Resisting temptation : the effect of an impulsive personality and a toxic environment on overeating
}

Citation for published version (APA):

Guerrieri, R. (2008). Resisting temptation : the effect of an impulsive personality and a toxic environment on overeating. [Doctoral Thesis, Maastricht University]. Datawyse / Universitaire Pers Maastricht. https://doi.org/10.26481/dis.20081204rg

Document status and date:

Published: 01/01/2008

DOI:

10.26481/dis.20081204rg

Document Version:

Publisher's PDF, also known as Version of record

\section{Please check the document version of this publication:}

- A submitted manuscript is the version of the article upon submission and before peer-review. There can be important differences between the submitted version and the official published version of record.

People interested in the research are advised to contact the author for the final version of the publication, or visit the DOI to the publisher's website.

- The final author version and the galley proof are versions of the publication after peer review.

- The final published version features the final layout of the paper including the volume, issue and page numbers.

Link to publication

\footnotetext{
General rights rights.

- You may freely distribute the URL identifying the publication in the public portal. please follow below link for the End User Agreement:

www.umlib.nl/taverne-license

Take down policy

If you believe that this document breaches copyright please contact us at:

repository@maastrichtuniversity.nl

providing details and we will investigate your claim.
}

Copyright and moral rights for the publications made accessible in the public portal are retained by the authors and/or other copyright owners and it is a condition of accessing publications that users recognise and abide by the legal requirements associated with these

- Users may download and print one copy of any publication from the public portal for the purpose of private study or research.

- You may not further distribute the material or use it for any profit-making activity or commercial gain

If the publication is distributed under the terms of Article $25 \mathrm{fa}$ of the Dutch Copyright Act, indicated by the "Taverne" license above, 


\section{Resisting temptation:}

The effect of an impulsive personality and a toxic environment on overeating 
Colophon

Cover art work by Tom Martin (www.newmoonilluminations.com)

Cover design by Hugo Alberts

(C) Copyright R. Guerrieri, Maastricht 2008

Universitaire Pers Maastricht

ISBN 978-90-5278-748-0

All rights reserved. No part of this book may be reproduced or transmitted in any form or by any means, without written permission from the author or, when appropriate, from the publisher of the article. 


\section{Resisting temptation:}

The effect of an impulsive personality and a toxic environment on overeating

\section{PROEFSCHRIFT}

ter verkrijging van de graad van doctor aan de Universiteit Maastricht, op gezag van Prof. mr. G.P.M.F. Mols, Rector Magnificus, volgens het besluit van het College van Decanen, in het openbaar te verdedigen op donderdag 4 december 2008 om 12.00 uur

door

Ramona Guerrieri

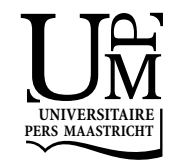


Promotor

Prof. dr. A. T. M. Jansen

Copromotor

Dr. C. Nederkoorn

Beoordelingscommissie

Prof. dr. M. L. Peters (voorzitter)

Prof. dr. H. J. Hospers

Prof. dr. C. Kemner (Universiteit Utrecht / Universiteit Maastricht)

Prof. dr. G. Pieters (Katholieke Universiteit Leuven)

Prof. dr. J. C. Seidell (Vrije Universiteit Amsterdam) 


\section{Contents}

Chapter $1 \quad$ General Introduction

Part I: Impulsivity as a cause of overeating

Chapter 2 The influence of trait and induced state impulsivity on food intake in normal-weight women

Chapter 3 Inducing impulsivity leads restrained eaters into overeating, whereas current dieters stick to their diet

Part II: The interaction between an impulsive personality and the toxic environment: its effect on overeating

Chapter $4 \quad$ How impulsiveness and variety influence food intake in a sample of healthy women

Chapter 5 The interaction between impulsivity and a varied food environment: Its influence on food intake and overweight

Chapter 6 General Discussion

Summary

Samenvatting

References

Dankwoord 



\section{Chapter 1}

\section{General Introduction}

Part I of the introduction (Impulsivity) is in press as:

Guerrieri, R., Nederkoorn, C., Jansen, A. (in press). The effect of an impulsive personality on overeating and obesity: Current state of affairs. Psychological Topics.

Part II of the introduction (The toxic environment) is published as:

Guerrieri, R. (2005). Toen de koekjestrommel de fruitschaal verdrong... De toxische omgeving als oorzaak van obesitas. [The toxic environment as a cause of obesity] De Psycholoog, 40, 642-646. 
Weight problems have become a hot topic when it comes to public health. Weight status is generally assessed by the Body Mass Index (BMI), calculated by dividing one's weight $(\mathrm{kg})$ by one's height $(\mathrm{m})$ squared. A BMI between 18.5 and 24.9 constitutes a normal weight, whereas a BMI ranging from 25 to 29.9 is defined as overweight and a BMI of 30 or more as obesity. The last decades have been hallmarked by a significant increase of overweight and obesity among children and adults. In 2003, the World Health Organisation (WHO) even declared obesity a global epidemic. In the United States for example $65 \%$ of the population is overweight or obese (Hedley et al., 2004). In the Netherlands overweight and obesity are also winning ground. In children between 4 and 16 years of age $13 \%$ are overweight and $3 \%$ are obese (van den Hurk, van Dommelen, van Buuren, Verkerk, \& HiraSing, 2007). This is a considerable increase compared to the 1980 overweight and obesity rates of $5 \%$ and $0.35 \%$, respectively (van den Hurk et al., 2007). In adults overweight and obesity rates have also risen steeply to $46.5 \%$ and $11 \%$, respectively (Central Bureau for Statistics, 2006). It should be noted that the figures for adults are based on self-report, which makes it likely that they are an underestimation of the scale of the problem (Visscher, Viet, Kroesbergen, \& Seidell, 2006).

If this trend continues, medical costs due to secondary health problems of overweight such as hypertension, diabetes and certain cancers will soar (Hu, 2003; Lean, 1998). At this moment an estimated $9 \%$ of medical expenditures are attributable to overweight and obesity in the United States (Flegal, 2005). Besides the obvious healthrelated consequences, psychological and social aspects of life are also seriously affected in obese individuals. Psychologically, these individuals suffer from a negative body image (Stunkard \& Sobal, 1995) and they often exhibit symptoms of depression (Werrij, Mulkens, Hospers, \& Jansen, 2006). Socially, obese individuals have to deal with prejudice and stigmatization (Stunkard \& Sobal, 1995).

It is clear that something needs to be done to stop or even reverse the obesity epidemic. In effect, the mechanism that causes weight gain is quite straightforward: weight gain is caused by a positive energy balance. In other words, energy intake through food supersedes energy expenditure through metabolism, thermo genesis and physical activity. By the same reasoning, weight loss could be achieved by lowering food intake and thus energy intake and/or increasing physical activity and thus energy expenditure. Consequently, weight loss interventions that focus on modifying energy intake and energy expenditure to create a negative energy balance should be successful. However, people seem to have great difficulties doing just that. Research has shown that lifestyle change 
programs often do lead to weight loss in the short term, but that attempts to maintain this weight loss in the long term are not very successful (Lowe, 2003; Hill, Thompson, \& Wyatt, 2005).

Due to growing overweight and obesity rates and a lack of effective interventions, much research on causal and maintaining factors of obesity has been done. Genetic, biological, psychological, sociocultural and ecological factors have all been suggested (Drewnowski, 1991), but research on genetics and biological factors seems to be overrepresented. For example, a search in Medline and Psychinfo with the terms 'obesity and genetics' and 'obesity and metabolism' yield 46.635 hits. However, the terms 'obesity and psychology' and 'obesity and environment' result in a mere 10.919 hits. Nonetheless, there is reason to believe that psychological and environmental factors are more adept at elucidating the causes of the recent obesity epidemic than genetic and biological factors. A recent review of research on eating behaviour in healthy humans has suggested that human interactions with environmental variables are more suited to determine the regulation of food intake than biological variables (Levitsky, 2005). For example, humans do not make accurate changes in their caloric intake to adjust for changes in the energy density of foods, but their caloric intake is influenced substantially by environmental factors such as variety and portion size (Levitsky, 2005). When one thinks about it, this finding is not so surprising. Varied, palatable food is assumed to activate a system called the hedonic system. This system promotes food intake due to environmental or emotional reasons. It complements a system called the homeostatic system, which promotes food intake due to energetic needs (Lowe \& Levine, 2005). Having a hedonic system is an evolutionary advantage (Blundell \& Gillett, 2001): in prehistoric times people had a better chance of survival if they could eat in the absence of physiological hunger when confronted with food. This enabled them to build up reserves in order to face periods of food shortage. It is plausible that in the current times of abundance of food this past virtue has become a vice. In this sense environmental factors can definitely contribute to our understanding of what is causing the current obesity epidemic.

However, not everyone who is faced with an abundance of food becomes overweight or obese. Some people remain lean despite the temptations in our environment. This is where individual differences come into the picture: a person's reaction to the environment is moderated by certain personality traits or other psychological factors that they do or do not possess (Blundell et al., 2005). One such psychological factor is impulsivity. There is reason to believe that impulsivity plays a role in the etiology and/or main- 
tenance of obesity. For example, higher levels of impulsivity have been found among the obese (e.g. Chalmers, Bowyer, \& Olenick, 1990) and impulsivity seems to be an obstacle in the treatment of obesity (e.g. Nederkoorn, Jansen, Mulkens, \& Jansen, 2007). Moreover, in a general population sample, consisting of normal- weight, overweight and obese women, impulsivity was positively associated with overeating and the preference for sweet and fatty foods, and these two factors were, in turn, positively related to BMI (Davis, Strachan, \& Berkson, 2004b). Although this research links impulsivity to overeating and obesity, the precise nature of the relationship is still unclear.

The current dissertation is concerned with helping to tip the scales from genetic and biological factors to psychological (impulsivity) and environmental (the current abundance of food) factors when it comes to research into the causes of the current obesity epidemic.

This general introduction aims to define the concept of impulsivity and the aspects of the 'toxic' environment, and to clarify how these factors have been linked to overeating and obesity in previous research. Moreover, reasons for expecting an interaction between impulsivity and the toxic environment are discussed. Lastly, the hypotheses and research goals of this dissertation are outlined.

Impulsivity

Definition and measurement

Generally impulsivity is defined as the tendency to think, control and plan insufficiently, which mostly results in an inaccurate or maladaptive response (Solanto et al., 2001). For example, if one is unable to plan one's meals, one's refrigerator might not contain the necessary ingredients to prepare a healthy meal, which could lead to the choice of an unhealthy snack.

Impulsivity is considered a multidimensional construct (e.g. Whiteside \& Lynam, 2001) for two reasons. First, correlations between self-report measures and behavioural measures of impulsivity are generally weak (e.g. Carrillo-De-La-Peña, Otero, \& Romero, 1993; Wingrove \& Bond, 1997). This could mean that self-report questionnaires and behavioural tasks measure a different aspect of impulsivity. Second, even within the behavioural tasks different operationalisations and explanatory models of impulsivity are used and these often intercorrelate poorly (e.g. Marsh, Dougherty, Mathias, Moeller, \& Hicks, 2002).

Although it is possible that weak intercorrelations between measures point to en- 
tirely different constructs, for most researchers these findings confirm that impulsivity is an umbrella concept. This means that it is a grouping of related concepts or models. This makes impulsivity research interesting, but at the same time rather complicated. One cannot simply refer to the relationship between impulsivity and overeating. The term 'impulsivity' can refer to multiple concepts and some of these concepts might be related to overeating, whereas others may not. Not distinguishing between all these aspects of impulsivity might lead to the premature conclusion that research on impulsivity and overeating is riddled with inconsistent findings. To avoid this, it is important that we do distinguish between different aspects of impulsivity.

Roughly, one can distinguish three sorts of operationalisations of impulsivity: insufficient response inhibition/premature responding, sensitivity to reward and selfreported trait impulsivity.

\section{Impulsivity as response inbibition or premature responding}

One possibility is to define impulsivity either as premature responding (i.e. responding before all relevant information is processed) or as insufficient response inhibition (i.e. a diminished ability to inhibit an already initiated response). Response inhibition models seem to be more widespread than premature responding models.

A popular response inhibition model is that of Logan, Schachar, and Tannock (1997). In this model, based on the executive-control model (Logan \& Cowan, 1984), impulsivity is seen as the diminished ability or inability to inhibit a prepotent or predominant response. According to the executive-control model (Logan \& Cowan, 1984) inhibition is a top-down process: a higher-order executive system interacts with a subordinate system. The executive system forms intentions, but the actual operations that are necessary to act out these intentions are carried out by the subordinate system. The subordinate system is dependent upon the executive system in the sense that the executive system delivers its resources. In case of a change in intentions or goals, the executive system can inhibit the subordinate system by ceasing to deliver resources. Consequently, the subordinate operations to act out the (previous) intention are stopped and in some cases reorganised to fit a new intention (Logan \& Cowan, 1984; Band \& van Boxtel, 1999). Individual differences in the ease with which the executive system can inhibit the subordinate system reflect individual differences in impulsivity. Logan (Logan \& Cowan, 1984; Logan et al., 1997) proposed the stop signal task as a paradigm to study inhibitory control. In the stop signal task participants perform a choice reaction-time task (e.g. press left in 
case of an $\mathrm{O}$ and right in case of an X). However, in 25\% of the cases the stimulus is accompanied by a stop signal (e.g. an auditory stimulus) and participants should not respond to the presented stimulus. The delay between the onset of the stimulus and the onset of the stop signal is varied dynamically so that inhibition is sometimes relatively easy (short delay) and sometimes relatively difficult (long delay). The more difficulties participants experience in inhibiting their response in the case of a stop signal, the more impulsive they are believed to be.

Other tasks that measure response inhibition, and that are based on the stop signal task, are the go/no-go task (Band \& van Boxtel, 1999) and the stop-change task (Band, van der Molen, Overtoom, \& Verbaten, 2000). The go/no-go task is a simplified version of the stop-signal task. There is typically no delay between the onset of the stimulus and the onset of the stop signal, which makes it easier to inhibit responses. The stop-change task differs from the stop signal task in the sense that the stop signal does not signal nonresponse, but the opposite response than the one indicated by the stimulus (e.g. instead of pressing right in case of an X, a participant should press left in case of a stop-change signal). In this task selective inhibition is necessary, whereas nonselective inhibition suffices in the stop signal task (Band et al., 2000). Consequently, this task is more difficult compared to the stop signal task. It should be noted that the difference in performance of response inhibition tasks between high and low impulsives is limited to the inhibition part of the task. Go reaction times typically do not differ between groups.

The Immediate and Delayed Memory Tasks (IMT/DMT; Dougherty et al., 1999) operationalise impulsivity not as insufficient response inhibition, but as premature responding. In the IMT 5-digit numbers are presented successively. The participant is instructed to react only when two identical numbers are presented. In the DMT the 5-digit numbers are separated by a distracter (12345). In both tasks catch trials are presented in which four of the five digits match the number that was previously presented. A response to such a catch trial constitutes a commission error, and the more commission errors are committed, the more impulsive a person is believed to be (Dougherty et al., 2003).

In sum, insufficient response inhibition is generally measured via three behavioural tasks: the stop signal task, the go/no-go task, and the stop-change task. The stopchange task is the most difficult task, followed by the stop signal task and the go/no-go task. The Immediate and Delayed Memory Tasks measure impulsivity as premature responding. 


\section{Impulsivity as sensitivity to reward}

A second possibility is to define impulsivity as sensitivity to reward. Impulsivity is operationalised as delay of gratification (e.g. Metcalfe \& Mischel, 1991), delay discounting (e.g. Reynolds, 2006) or reward-sensitivity (e.g. Gray, 1987; Davis et al., 2004b).

Delay of gratification refers to a task in which the willpower of the participant, usually a child, is put to the test. The child is confronted with a smaller and a larger reward or a less or more preferred reward. When the experimenter leaves the room, the child is faced with the choice between two options. Either the child waits for the experimenter to return and hand out the larger or more preferred award, or the child rings a bell and the experimenter returns directly to hand out the smaller or less preferred reward (Metcalfe \& Mischel, 1999). Not being able to delay the reward although the option of waiting for the larger reward is clearly preferred, is seen as impulsive behaviour. This kind of behaviour is thought to arise out of a dominance of the hot system over the cool system (Metcalfe \& Mischel, 1999). The hot system is noncognitive, emotional, and stimulus-controlled. This system is present at birth and it may be situated in the amygdala. The cool system is cognitive, emotionally neutral, and strategic. It is thought to develop later in life and it may be situated in the hippocampus and prefrontal regions in the brain. Early in life, the hot system dominates, but with aging the cool system gains strength. Individual differences in the balance between hot and cool behaviour reflect individual differences in impulsivity.

Delay discounting tasks usually consist of numerous trials in which participants make hypothetical choices between larger, but delayed monetary amounts and smaller, but immediate monetary amounts (Reynolds, 2006). The measure can be question-based and hypothetical, involving imaginary delays and rewards, or it can be a 'real time' measure in which participants experience the actual delays and rewards that follow from the choices that they make. Although the latter measure seems to be more ecologically valid, it seems to give rise to findings that are comparable with the question-based measure. Moreover, these measures correlate positively and significantly (Reynolds, 2006). In delay discounting it is assumed that the subjective value of a certain reward devaluates with increasing delay. The steeper the devaluation curve, the sooner participants shift from preferring larger/later amounts to smaller/sooner amounts, and the more impulsive there are believed to be (Reynolds, 2006).

Delay of gratification and delay discounting differ in the sense that the secondchoice reward can still be chosen when the delay is too much to bear in the delay of 
gratification paradigm, whereas in delay discounting one cannot reconsider one's decision. These tasks are therefore believed to measure different aspects of delay-related impulsivity. Delay discounting is thought to represent choice preference, whereas delay of gratification measures whether one is able to sustain that choice (Reynolds \& Schiffbauer, 2005). For example, a person who wants to lose weight, should first make the choice to restrict his or her caloric intake, and should then sustain that choice by resisting palatable high-calorie foods (Reynolds \& Schiffbauer, 2005).

Reward-sensitivity is a construct that stems from addiction research. Since researchers have noticed parallels between overeating and nicotine, alcohol and drug addictions, the term is increasingly used in eating related research (Davis \& Claridge, 1998; Orford, 2001). Reward-sensitivity is linked to a heightened dopamine availability in the mesocorticolimbic pathway in our brains (Davis \& Fox, 2008). Reward-sensitive people detect more rewarding stimuli and are more likely to approach these rewarding stimuli (Davis \& Fox, 2008). This is very closely related to what Gray (1987; Avila, 2001) termed the Behavioural Activation System (BAS). The BAS is activated by signals of reward and non-punishment and is responsible for appetitive behaviour or impulsivity. According to Gray (1987) the BAS is complemented by the BIS or Behavioural Inhibition System. The BIS is activated by signals of punishment and non-reward and is responsible for aversive behaviour or anxiety (Avila, 2001). BAS activation or reward-sensitivity is usually measured with one of two self-report measures: the BAS scale of the BIS/BAS scales (Carver \& White, 1994) or the Sensitivity to Reward (SR) scale of the Sensitivity to Punishment and Sensitivity to Reward Questionnaire (SPSRQ; Torrubia, Avila, Molto, \& Caseras, 2001). The BAS scale consists of three subscales: Drive (pursuit of goals), Fun Seeking (approach behaviour towards new and potentially rewarding stimuli), and Reward Responsiveness (the positive reaction to signals of reward). The SR scale consists of 24 items evaluating approach behaviour and its motivating and pleasurable effects (Davis \& Fox, 2008). In children's research, like in Chapter 5 of this dissertation, a behavioural task is mostly used: the door opening task (Nederkoorn, Braet, Van Eijs, Tanghe, \& Jansen, 2006a; Matthys, van Goozen, de Vries, Cohen-Kettenis, \& van Engeland, 1998). In this task the participant can earn points. The goal of the task is to earn as many points as possible and then stop the game. On the computer screen the child sees a door that can be opened by pushing a button. This door can reveal a happy face, which means that the child has won one point. However, the door can also reveal a sad face, which means that the child loses one point. There are 100 doors in total. The computer determines whether 
a door hides a happy or a sad face, but the chance of encountering a happy face diminishes with every ten doors that are opened. This means that in the beginning most of the doors hide happy faces, but the more doors are opened, the less happy faces the child encounters. At a certain moment the child will experience more losses than gains and it should stop the game. The longer a child continues to open doors in search of wins despite all the losses, the more sensitive to reward it is believed to be. A task that also measures response perseveration and that can also be used with adults, is the card playing task (Siegel, 1978). The card arranging reward responsitivity objective test (CARROT) measures the extent to which participants speed up their performance of a simple card sorting task when offered a small financial reward (Powell, Al-Adawi, Morgan, \& Greenwood, 1996). Last, the Iowa Gambling Task requires participants to make a series of card selections. They can choose from four decks. Deck A and B yield high gains, but even higher losses, resulting in a total loss. Deck C and D, on the other hand, yield smaller gains, but also small losses, resulting in a total gain. It other words, the most sensible choice in the long run is to select cards from decks C and D (Bechara, Damasio, Damasio, \& Anderson, 1994; Bechara \& Damasio, 2002). The harder it is for participants to switch from decks $\mathrm{A}$ and $\mathrm{B}$ to $\mathrm{C}$ and $\mathrm{D}$, the more impulsive they are believed to be.

In sum, reward-related impulsivity is measured with different paradigms that measure slightly different things. The delay discounting procedure measures whether one is capable of making a 'sensible' choice in the sense of choosing a larger later reward over a smaller sooner reward. The delay of gratification paradigm measures whether one is capable of sustaining that choice. Reward-sensitivity is a construct that stems from addiction research. It is linked to a heightened dopamine availability in our brains. It can be measured both via self-report (BIS/BAS, SPSRQ) and via behavioural tasks (door opening task, card playing task, CARROT, Iowa Gambling Task).

\section{Impulsivity as a self-reported personality trait}

In personality research, the aim is to clarify the construct of impulsivity, mostly by linking it to major personality systems. This line of work has yielded a number of selfreport measures of impulsivity like the $\mathrm{I}_{7}$ Impulsiveness Questionnaire (Eysenck, Pearson, Easting, \& Allsopp, 1985), the Dickman Impulsivity Inventory (DII; Dickman, 1990), the Barratt Impulsiveness Scale (BIS; Patton, Stanford, \& Barratt, 1995), and the UPPS Impulsive Behavior Scale (Whiteside \& Lynam, 2001).

Eysenck (1990) sees personality as the interaction between three central traits: ex- 
traversion, neuroticism, and psychoticism. Extraversion is linked to a low inner arousal level. This leads extraverted people to seek stimulation to raise their arousal level. People with high inner arousal do not need external stimulation and are seen as introvert. People high in neuroticism are seen as emotionally unstable whereas people low in neuroticism are seen as being emotionally stable. Psychotic people tend to be aggressive, cold, egocentric, and impulsive. Impulsivity is seen as a lower order trait of psychoticism, but another component of impulsivity seems to be venturesomeness, a lower order trait of extraversion. The $I_{7}$ was developed to measure impulsivity as impulsiveness (a lower order trait of psychoticism) and venturesomeness (lower order trait of extraversion). The impulsiveness scale consists of 19 yes/no items such as 'Do you usually think carefully before doing anything?' or 'Do you often change your interests?'. The venturesomeness scale consists of 16 yes/no items such as 'Would you enjoy water skiing?' or 'Do you sometimes like doing things that are a bit frightening?'.

Dickman (1990) approached the construct of impulsivity from a different point of view. He defined impulsivity as 'the tendency to deliberate less than most people of equal ability before taking action' (pp. 95). However, he also contended that the consequences of deliberating less are not necessarily negative. In some situations it is advantageous to respond rapidly without much deliberation. This led Dickman to explore whether reacting impulsively when this is advantageous (functional impulsivity) is different from reacting impulsively when this is nonadaptive (dysfunctional impulsivity). This resulted in the Dickman Impulsivity Inventory, consisting of two subscales. The dysfunctional impulsivity scale has 12 true/false items such as 'I often get into trouble because I don't think before I act', whereas the functional impulsivity scale consists of 11 true/false items such as 'People have admired me because I can think quickly'.

Barratt (Patton et al., 1995) sees impulsivity as consisting of three aspects: a motor aspect, an attentional aspect, and a planning aspect. The Barratt Impulsiveness Scale (Patton et al., 1995) was developed to measure each of these aspects and thus consists of three subscales: motor impulsiveness (acting without thinking), attentional impulsiveness (not focusing on the task at hand, cognitive instability) and nonplanning impulsiveness (lack of orientation to the future).

Most recently, Whiteside and Lynam (2001) attempted to clarify the construct of impulsivity. They made use of the Five Factor Model of personality (FFM; McCrae \& Costa, 1990). The FFM defines personality as the combination of five broad factors: neuroticism, extraversion, openness to experience, agreeableness, and conscientiousness. It 
was hypothesized that four lower order facets of three broad factors are related to impulsivity: impulsiveness (neuroticism), excitement seeking (extraversion), self-discipline and deliberation (conscientiousness). Factor analysis on self-report impulsivity data of 437 undergraduates indeed revealed four factors, labelled urgency, (lack of) premeditation, (lack of) perseverance, and sensation seeking. Scales for each factor were created to form the UPPS Impulsive Bebavior Scale. The urgency scale is most closely tied to the impulsivity facet and contains 12 items such as 'When I am upset I often act without thinking'. The 11 (lack of) premeditation items such as 'I am a cautious person' are most related to deliberation. The 10 (lack of) perseverance items such as 'Unfinished tasks really bother me' are a translation of the self-discipline facet, and the counterpart of the excitement seeking facet is the sensation seeking scale with 12 items such as 'I will try anything once'.

In sum, the construct of impulsivity has been clarified by situating it within two major personality systems. This resulted in the $I_{7}$, based on Eysenck's Three Factor Model, and the UPPS Impulsive Behavior Scale, based on the Five Factor Model of personality. Two other frequently used self-report measures are the Dickman Impulsivity Inventory (DII) and the Barratt Impulsiveness Scale (BIS).

\section{Conclusion}

First, in reading the overview of impulsivity above it should be noted that this particular classification of impulsivity is artificial and cannot be seen as static because some operationalisations of impulsivity fit in multiple models. For example, the BIS/BAS account of impulsivity can be seen as a reward-sensitivity model, but it can also be characterized as a trait model of impulsivity. Moreover, this overview of models and operationalisations of impulsivity is not exhaustive. For example, biologically based measures such as event-related potentials (see Moeller, Barratt, Dougherty, Schmitz, \& Swann, 2001) are not included. The objective was to include all models and operationalisations of a psychological nature that are frequently referred to (see Table 1.1 for an overview).

The fact that impulsivity seems to be such a broad concept that is measured in so many different ways, renders the choice of an impulsivity measurement very difficult. Theoretical considerations should primarily guide the choice of a particular impulsivity measure. These theoretical considerations come in the form of specified hypotheses. It is not sufficient to hypothesize that 'impulsivity causes overeating'. One should consider to what extent each aspect of impulsivity can play a significant role. In this case it was, for 
example, theoretically more warranted to hypothesize that sensitivity to reward and insufficient response inhibition would play a significant role, than that risk taking or adventuresomeness would be important.

However, even after having made this choice, one needs to make at least two more. First, one should choose between self-report and behavioural measures. Second, even within self-report and behavioural measures, more than one option is available and one needs to consider which option fits one's needs best. For the first choice, pragmatic and common-sense comparison of self-report versus behavioural measures can help. Self-report measures, for example, are quick and easy to administer, and ask participants' judgement of 'real life' situations. The disadvantage of such measures is that one needs to avoid social desirability and rely on the participants' candour. These problems are lacking in behavioural tasks, which in their turn are less ecologically valid and take up more time and equipment to administer. Once the choice between self-report and behavioural tasks is made, one needs to choose one measure within the self-report or behavioural measures that are available. Normally one would assess the quality of a measure based on its reliability and validity. However, this information is largely lacking for impulsivity measures. It is thus of great importance that future research brings clarity in this issue. Until then our best bet is to rely on previous research and experiences.

In the current dissertation a variety of impulsivity measures was chosen. Rewardsensitivity (door-opening, BIS/BAS), insufficient response inhibition (stop signal task), and trait impulsivity measures (BIS, $\mathrm{I}_{7}$ ) were all used. The choice of these measures was based on previous eating related research that made use of these measures (Claes, Vandereycken, \& Vertommen, 2002; Claes, Nederkoorn, Vandereycken, Guerrieri, \& Vertommen, 2006; Nederkoorn, Van Eijs, \& Jansen, 2004; Nederkoorn et al., 2006a; Nederkoorn, Smulders, Havermans, Roefs, \& Jansen, 2006b; Nederkoorn et al., 2007)

\section{Research on impulsivity and overeating}

One can imagine that all three kinds of impulsivity contribute to the obesity epidemic. First, when it comes to response inhibition, the confrontation with palatable food might inevitably trigger the prepotent response to eat it. In times when food was scarce this was an adaptive response (Blundell \& Gillett, 2001). However, it is feasible that in today's obesogenic environment not being able to inhibit one's prepotent responses contributes significantly to the problem of obesity. Second, when it comes to rewardsensitivity, momentary craving might be more important than future goals of losing 
weight. Moreover, reward-sensitivity might lead people to make the wrong food choices: they might prefer foods that are sweet and fat because palatable food has a greater rewarding value than bland food (Davis et al., 2007). Third, when it comes to impulsivity as a personality trait, lacking the ability to plan meals (nonplanning impulsivity BIS) for example, might lead to more snack food consumption and hence to overweight or a lack of perseveration (UPPS Impulsive Behavior Scale) might make it difficult to stick to an intention to eat healthily.

Research in populations that typically overeat (the obese and Bulimia Nervosa patients), in populations that are typically impulsive (e.g. Attention Deficit Hyperactivity Disorder patients) and in healthy participants has indeed begun to indicate that impulsivity plays a role in the problem of overeating.

Table 1.1

Overview of psychological impulsivity models and measures

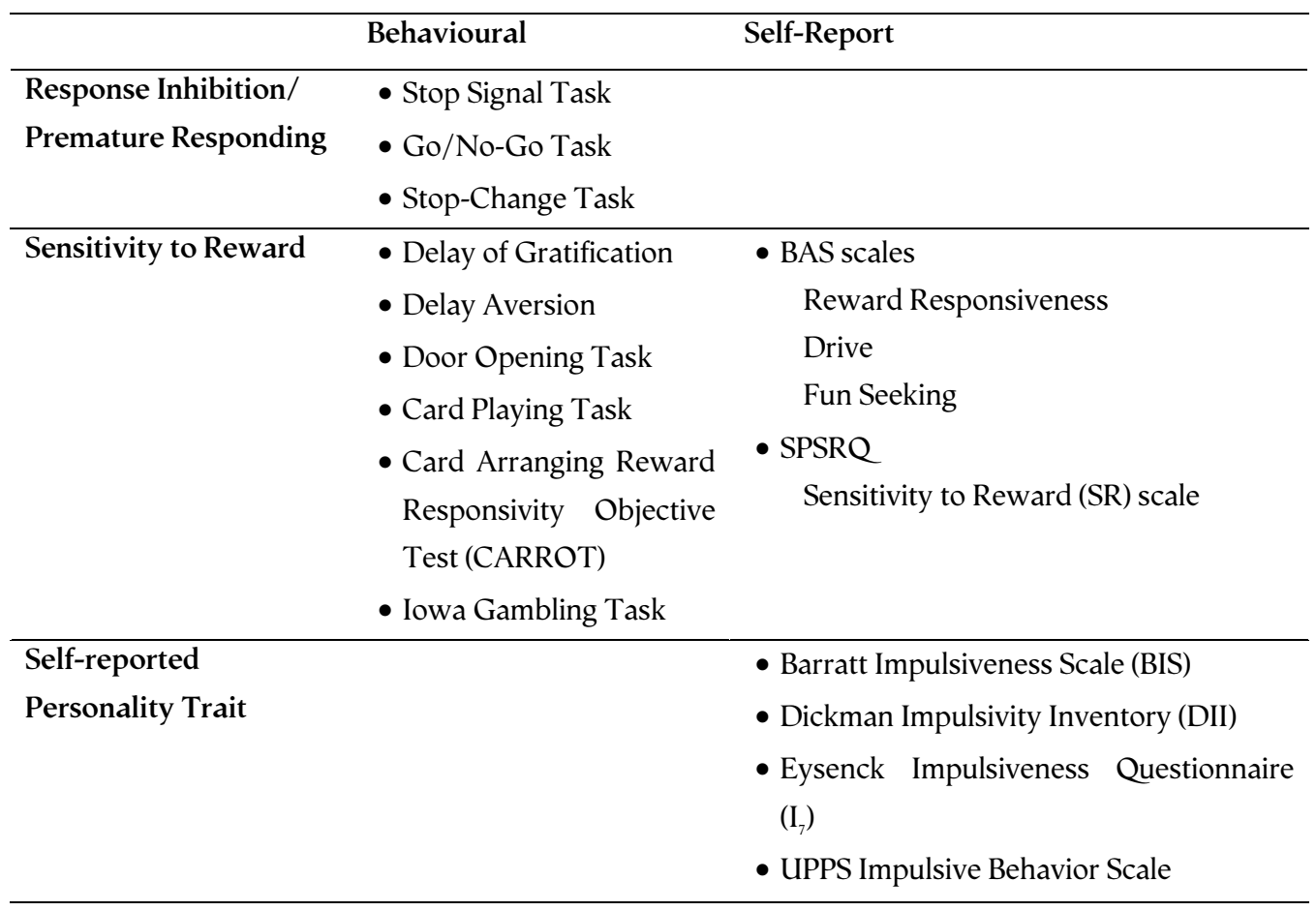




\section{Impulsivity and obesity}

Research using clinically obese populations has often demonstrated heightened impulsivity in the obese. For example, comparison in personality patterns through selfreport between obese participants and their normal-weight matched controls revealed that the obese participants scored higher on the impulsivity facet of novelty seeking and lower on the facets of planning and persistence (Chalmers et al., 1990). Moreover, within the obese population, the more severely obese are more impulsive compared to the less severely obese. Rydén et al. (2003) reported that their severely obese participants, candidates for gastric banding, scored significantly higher on a self-report measure assessing the tendency to act on the spur of the moment and to make decisions rapidly compared to less obese, conventionally treated participants. Another more severe group within obese patients are Binge Eating Disorder (BED) patients. They exhibit more eating pathology and more psychopathology compared to obese patients without eating binges (Fassino, Leombruni, Pierò, Abbate-Daga, \& Rovera, 2003). Nasser, Gluck, \& Geliebter (2004) found that BED patients scored significantly higher on a self-report measure of general impulsiveness compared to controls. They also found positive significant correlations ( \pm .50$)$ between participants' impulsivity score and the BED criteria 'Loss of control during a binge' and 'Eating when not physically hungry'.

Davis and colleagues conducted a line of research in which they gathered selfreport data on sensitivity to reward (SPSRQ and BAS subscale of the BIS/BAS scales), food preferences, and overeating in women ranging from normal weight to obese. They tested whether sensitivity to reward could lead to overeating and food preferences for sweet and fatty foods, which in turn could lead to a higher BMI (Davis et al., 2004b; Davis et al., 2007). Using structural equation modelling this model was confirmed (Davis et al., 2007). Sensitivity to reward was linked positively to overeating and preferences for food high in sugar and fat, and overeating and food preferences were in turn linked positively to BMI. However, in one study the relationship between sensitivity to reward and BMI was not linear (Davis et al., 2004b). More precisely, among overweight participants the association was positive, as expected, but among the obese the association reversed into a negative one. In other words, the more obese, the less sensitive to reward. These results were replicated in a sample with a mean BMI of 29.5 (Davis \& Fox, 2008) and interpreted as evidence for the hypothesis that long-term overeating, fostered by a hyperactive reward system, might lead to downregulation of this system due to overstimulation. Of course, sensitivity to reward was measured via self-report, which could foster socially desirable answers in the obese. In order to rule out this alternative explanation of the 
findings, sensitivity to reward should be measured with a behavioural task such as the card arranging reward responsitivity objective test (CARROT).

Besides self-report impulsivity, obese populations also differ in their performance of response inhibition and reward-directed impulsivity tasks. Nederkoorn and colleagues found that obese women and children were impaired in general response inhibition as measured by the stop signal paradigm compared to control participants (Nederkoorn et al., 2006a, Nederkoorn, Smulders, Havermans, Roefs, \& Jansen, 2006b). In other words, even on a very basic motoric level that has nothing to do with food the obese children were less able to inhibit their responses. Moreover, impulsivity turned out to be an obstacle in the treatment of the obese children: the children that were worst at inhibiting responses, lost less weight (Nederkoorn et al., 2006a; Nederkoorn et al., 2007).

Nederkoorn et al. (2006a) also found that the obese children were more sensitive to reward during the door opening task compared to control children. Moreover, among the obese children, the children with eating binges were more sensitive to reward compared to the obese children without eating binges. In an earlier study by Davis and colleagues, with participants with a BMI ranging from 17.3 to 45.4, the Iowa Gambling Task was used. Participants with a BMI $<25$ showed an improvement in good decisions across trials, whereas participants with a BMI $\geq 25$ showed no improvement across trials (Davis, Levitan, Muglia, Bewell, \& Kennedy, 2004a). Research using the delay of gratification paradigm has shown that obese children have difficulties with delay of gratification tasks only when the incentive is edible (Bonato \& Boland, 1983). This suggests that food could be especially rewarding for the obese. This has indeed been found. In a study by Saelens and Epstein (1996) obese participants chose to work for food instead of sedentary activities more often than controls.

In sum, overweight and obese people seem more sensitive to reward and less adequate at inhibiting prepotent responses, even on a very basic motoric level that has nothing to do with food. Moreover, they report to seek more novelty, to plan less efficiently, to be less persistent, and to act on the spur of the moment.

\section{Impulsivity and Bulimia Nervosa}

Not only the obese overeat. Bulimia Nervosa (BN) and Anorexia Nervosa (AN) binge/purge subtype patients are also characterized by a specific type of overeating, binge eating. If impulsivity plays a role in binge eating, then $\mathrm{BN}$ and $\mathrm{AN}$ binge/purge patients should score higher on measures of impulsivity compared to healthy controls, and 
possibly AN restricters. This is exactly what has been demonstrated with self-report measures as well as with response inhibition and reward-related tasks.

Davis \& Woodside (2002) compared BN patients to AN restricters and demonstrated that AN restricters scored well below the age-matched norm scores for selfreport reward-sensitivity, whereas $\mathrm{BN}$ patients scored above these norms. Rosval et al. (2006) demonstrated that BN and AN binge/purge patients scored significantly higher on attentional impulsiveness, but did not differ from controls in their scores on the BIS/BAS scales, the $I_{7}$, and the other subscales of the BIS (motor and nonplanning impulsiveness). In another study BN patients scored significantly higher than AN patients - no information about subtypes was given - on the $\mathrm{I}_{7}$ questionnaire (Fahy \& Eisler, 1993). Claes et al. (2002) also demonstrated that $\mathrm{I}_{7}$ scores were significantly higher in BN compared to AN patients, with only the differences between $\mathrm{BN}$ and $\mathrm{AN}$ restricters reaching statistical significance. Claes et al. (2006) mainly found significantly less self-report impulsivity in AN restricters compared to controls, BN and AN binge/purge patients.

As far as behavioural tasks are concerned, BN and AN binge/purge patients inhibited significantly less during the go/no-go task (Rosval et al., 2006), and BN patients scored higher than controls on reward-sensitivity as measured by the CARROT (Kane, Loxton, Staiger, \& Dawe, 2004). However, Claes et al. (2006) did not find any differences on stop signal task performance between eating-disordered participants and control participants.

Nederkoorn, van Eijs, and Jansen (2004) had a somewhat different approach. They recruited healthy women who scored high on the Restraint Scale (RS; Herman \& Polivy, 1980), a measure of (unsuccessful) dieting, weight concerns and loss of control over eating that is predictive of bulimic symptoms (Stice, Ozer, \& Kees, 1997). Compared to low RS scorers, these women scored significantly higher on several self-report impulsivity measures such as the BIS and the BAS Reward Responsiveness subscale. Moreover, these women had more trouble inhibiting their responses during the stop signal task.

In sum, BN and AN binge/purge patients generally scored higher on impulsivity measures compared to AN restricters and healthy controls. The measures that were used contain trait impulsivity measures, reward-related measures and measures of insufficient response inhibition. Furthermore, healthy women, who are at risk for bulimic symptoms, scored higher on reward-related self-report measures and were less efficient in inhibiting their responses during the stop signal task than their controls. 


\section{Impulsivity and Attention Deficit Hyperactivity Disorder}

Obesity has also been linked to Attention Deficit Hyperactivity Disorder (AD/HD), a disorder that is hallmarked by an excess of impulsive behaviour. Disproportionate amounts of children with $\mathrm{AD} / \mathrm{HD}$ were found within a group of children who were hospitalized for severe obesity (Agranat-Meged et al., 2005). The reverse also turned out to be true: the mean $\mathrm{BMI}$ in a sample of $\mathrm{AD} / \mathrm{HD}$ boys was significantly higher than the age-adapted reference values (Holtkamp et al., 2004). In adults two studies demonstrated a significantly higher prevalence of $\mathrm{AD} / \mathrm{HD}$ in obese participants in treatment (Altfas, 2002; Fleming \& Levy, 2002). These studies provide indirect evidence for the hypothesis that the impulsive behaviour, characteristic of $\mathrm{AD} / \mathrm{HD}$, could lead $\mathrm{AD} / \mathrm{HD}$ patients to overeat. Overeating repeatedly could lead to overweight or obesity despite these patients' hyperactivity, which is also characteristic of $\mathrm{AD} / \mathrm{HD}$. This hypothesis was tested by Davis, Levitan, Smith, Tweed, \& Curtis (2006). They used selfreport questionnaires to measure the extent of $\mathrm{AD} / \mathrm{HD}$ symptoms during childhood and to assess impulsivity in a sample of healthy adult women. Using structural equation modelling they tested and found that $\mathrm{AD} / \mathrm{HD}$ symptoms related positively to overeating and that overeating, in turn, was correlated with a higher BMI.

In sum, $\mathrm{AD} / \mathrm{HD}$, a disorder hallmarked by impulsivity, has been linked to overweight and obesity: $\mathrm{AD} / \mathrm{HD}$ patients seem to have a higher $\mathrm{BMI}$ compared to healthy controls, and disproportionate amounts of $\mathrm{AD} / \mathrm{HD}$ patients were found among obese individuals in treatment. Furthermore, $\mathrm{AD} / \mathrm{HD}$ symptoms in childhood were linked to overeating.

\section{Impulsivity and eating attitudes and behaviour in bealthy participants}

Recently it has been shown that even in healthy, lean participants impulsivity is of importance when it comes to food. Loxton \& Dawe $(2001,2006,2007)$ conducted a number of studies in healthy participants in which they found a positive relation between reward sensitivity and dysfunctional eating (drive for thinness, bulimic symptoms). Their samples consisted of senior high school girls or female college students and sensitivity to reward was measured both with self-report questionnaires (SPSRQ and BAS subscale of the BIS/BAS scales) and with the CARROT, a reward-directed impulsivity task.

Moreover, healthy individuals who were more sensitive to reward according to a self-report questionnaire turned out to have more pronounced neural responses to images of appetizing food (Beaver et al., 2006). This could indicate that for high-impulsives 
it is harder to resist food than for low-impulsives. Whether this is really the case, is hard to determine based on current research, because actual food intake is rarely measured in studies on impulsivity. One exception is a study by Rotenberg at al. (2005) in which 'lack of control' thoughts were primed. These thoughts did indeed lead to greater food intake compared to priming 'control' thoughts. Lack of control might be different from impulsivity in the sense that it puts more emphasis on the cognition of powerlessness, whereas impulsivity might be more related to behavioural activating. However, the concepts do show a great overlap and the results of the study do support the view that induced lack of control might cause overeating, compared to induced control. Whether induced impulsivity could lead healthy participants into overeating was investigated in this dissertation.

\section{Conclusion}

Impulsivity in all its forms has repeatedly been linked to overeating, although there have been some null findings (e.g. Wonderlich, Connolly, \& Stice, 2003). The relationship between increased impulsivity and the problem of overeating is quite robust since it generally persists even when impulsivity is measured in different ways (trait impulsivity vs. response inhibition vs. sensitivity to reward). However, previous research mostly yielded indirect evidence, so that one cannot draw conclusions about causality. The associations that have been found are necessary, but insufficient to establish that impulsivity causes overeating. Impulsivity could lead to overeating, and hence to overweight. However, it is also possible that being overweight or obese could lead to more impulsive behaviour in the form of overeating. Furthermore, in order to establish a causal relationship, one needs to rule out the possibility of a third variable that influences impulsivity as well as overeating. In order to do this, one should randomly assign healthy participants to one of two groups: an experimental group in which impulsivity is manipulated experimentally versus a control group. If the experimental group shows a heightened food intake during a bogus taste test, then one could rightfully conclude that increased impulsivity caused the heightened food intake.

In sum, if one wishes to conclude that impulsivity causes a heightened food intake, one needs to affirmatively answer four questions. First, 'Are people who typically overeat (e.g. obese, BN and AN binge/purge patients) more impulsive compared to controls?'. The answer seems to be 'yes'. Second, 'Do people who are typically impulsive (e.g. $\mathrm{AD} / \mathrm{HD}$ patients) overeat?'. Although research is still fairly limited, the answer to this 
questions also seems to be 'yes'. Third, 'Do healthy participants with an impulsive personality have a heightened food intake, and possibly gain more weight in the long term, compared to their less impulsive counterparts? And fourth, 'Does manipulating impulsivity/inhibition in a group of healthy participants lead to an increased/decreased food intake compared to controls?. Except for one study actual food intake has not been measured and impulsivity has not been manipulated in impulsivity studies. Consequently, the last two questions, and consequently the causal question are left unanswered. It was one of the aims of the current project to make a start in answering these questions.

\section{The toxic environment}

Changes in our food environment

In addition to personality factors like impulsivity, environmental factors also influence human food intake. The quality and availability of food have increased through time without leading to an obesity epidemic. However, about two decades ago, our food environment started changing in another way: it became toxic. This means that especially sweet and fatty foods dominate the food market. This sort of food has never been more varied or easily accessible than it is today. McCrory et al. (1999) have demonstrated that the number of snacks, sweets, cookies and cakes that are introduced to the food market increase disproportionally compared to the available fruit and vegetables. Moreover, fizzy drinks, fast food en salty snacks are being offered in increasingly large - two to five times as large - portions, whereas healthy alternatives like milk are being offered in increasingly smaller portions (Nielsen \& Popkin, 2003; Nielsen \& Popkin, 2004; Kral \& Rolls, 2004). Besides variety, large portion sizes and availability, sweet and fatty foods contain more calories per gram and the price per calorie is much cheaper than the price of healthy calories in fresh fruit and vegetables (Drewnowski, 2004).

\section{How these changes affect our food intake}

Claiming that the environment plays an important role in the current obesity epidemic - or in any other problem - will surely give rise to sceptical reactions. Surely people are responsible for the choices they make, even if there are many temptations in our environment. In the realm of eating behaviour, this conviction seems not to be in accordance with the outcomes of scientific research. In general, participants tend to consume more food than control participants when the food is more varied, offered in large portions or more easily available. What is even more remarkable, is that they seem to do so 
outside of their own awareness.

In an early study participants consumed more ice-cream in the lab when they were offered three flavours compared to only their favourite flavour (Berry, Beatty, \& Klesges, 1985). Variety in structure instead of flavour led to the same result: participants consumed more pasta in different shapes compared to being offered only one shape of pasta (Rolls, Rowe, \& Rolls, 1982). The effect of variety in flavour and/or structure on food intake is a quite reliable one. In a review article nine out of ten experiments that reported food intake demonstrated a positive effect of variety on food intake. All sorts of food were used, ranging from yoghurt and sandwiches to cheeseburgers and pizza (Sørensen et al., 2003).

The mechanism that researchers believe to be at the basis of this effect is called Sensory Specific Satiety (SSS; Rolls et al., 1982). It has been demonstrated in the lab multiple times that participants rate the taste of food that they have just eaten as less pleasant compared to food that they have not eaten. Even in animals the same neurons that did not react to eaten food were found to react to food that had not been eaten (Rolls, Rolls, \& Rowe, 1983). It is this mechanism that ensures that being satiated with a main course does not rule out that one can still be tempted to have dessert.

Variety could play an important role in making our food environment toxic. Researchers find an association between variety in one's diet and one's fat mass. A larger variety in sweet and fatty foods is associated with a larger fat mass, whereas a larger variety in vegetables is associated with a smaller fat mass (McCrory et al., 1999). Another study showed that successful dieters vary less in healthy and unhealthy foods, compared to less successful dieters (Raynor et al., 2005). Variety seems to be an important aspect of the toxic environment, but more research is needed to determine its precise relationship with obesity and successful dieting.

Besides variety, manipulating the portion size also affects food intake. Visitors of a movie theatre consumed 53\% more popcorn when they were given a 240 g portion compared to half this portion. It should be noted that the participants who did not particularly like the popcorn were influenced just as much by the larger portion size as the participants who did like the popcorn (Wansink \& Park, 2001). The effect of portion size on food intake is not limited to snack food. Unsuspecting participants in a restaurant consumed $43 \%$ more of a pasta dish on days when $377 \mathrm{~g}$ portions were served compared to when the standard $248 \mathrm{~g}$ portion was served (Diliberti et al., 2004). In a more controlled, but less ecologically valid study participants consumed an afternoon snack and dinner in 
the lab on five consecutive days. The snack consisted of a bag of crisps of $28,42,85,128$, or 170 grams, served in a random order. As expected, snack consumption increased with portion size. Another important question was whether the participants would compensate for their heightened snack consumption by consuming less during the following mealtime: dinner. This turned out not to be the case. On the contrary, the larger the portion of crisps as an afternoon snack, the more food was consumed at dinner time (Rolls et al., 2004). This effect was confirmed by Kral \& Rolls (2004).

In sum, offering food in large portions leads to a heightened food intake. This effect has been demonstrated with both snacks and regular meals and it even occurs without people demonstrating a particular liking for the food that is offered. Moreover, people do not seem to compensate for the extra calories of larger portions during following meals.

Why does portion size have such a strong effect on food intake? When determining how much to eat, people seem to pay more attention to visual cues than to satiety signals coming from within. In other words, 'people use their eyes to count calories and not their stomachs' (Wansink, Painter, \& North, 2005, pp. 98). Wansink et al. (2005) offered their participants soup in regular bowls or in bowls that were ingeniously and unobtrusively refilled during eating. The second group consumed $73 \%$ more soup compared to the first group. Despite the overconsumption in the second group, these participants did not feel more full than the participants in the first group. Moreover, their estimation of the amount of consumed soup did not differ from the estimations of the first group.

Enlarging the portion size of the food that is offered is one way of making food more easily available. Another way is to make the food more accessible. This is what Painter, Wansink, \& Hieggelke (2002) did. They placed a box of chocolates in one of three locations: on top of the participant's desk, in their desk drawer, or on a shelf at $2 \mathrm{~m}$ distance of the desk. In the first condition the chocolates were easily accessible and visible, in the second condition the chocolates were easily accessible, but not visible, and in the third condition the chocolates were visible, but not easily accessible. Both visibility and accessibility significantly contributed to the number of chocolates consumed. When the chocolates were on their desk (both visible and accessible), participants consumed 2.9 chocolates more each day compared to when the chocolates were in their desk (accessible, but not visible) and 5.6 more than the participants who had to walk $2 \mathrm{~m}$ to get a chocolate (visible, but not easily accessible). In conclusion, accessibility and, to a lesser 
extent, visibility of food both contributes to a heightened intake of that food. In our toxic food environment where sweet and fatty foods in particular are accessible, this effect contributes to the current obesity epidemic.

In sum, both naturalistic and laboratory studies demonstrate that participants consume more food when this food is varied, when it is offered in large portion sizes and when it is easily accessible. In our current toxic food environment variety, enlarged portion sizes and easy accessibility are particularly characteristic of sweet and fatty foods. It is even more reason for concern that people do not seem to be aware of these environmental influences on their food intake and that they do not feel more satiated after consuming more calories. Moreover, one does not even have to demonstrate a particular liking for the food that is offered in order to be influence by certain environmental factors.

The interaction between an impulsive personality and the toxic environment

In the previous sections research has been described that links both impulsivity and the toxic environment to overeating. The remaining question is whether these factors interact to affect food intake. More precisely, does an impulsive personality leave one extra vulnerable to the toxic food environment and thus in particular danger of constant overeating?

This hypothesis has been formulated in the literature more than once. Blundell et al. (2005), for example, see a high food-induced pleasure response as a behavioural risk factor for overconsumption and they also predict that this risk factor will not lead to excessive food intake in a benign environment. In their opinion, it takes a toxic environment to lead these high-risk individuals into overeating. Furthermore, Davis et al. (2006) contend that poor inhibitory control could lead to unrestrained eating, especially in our toxic environment that offers unlimited access to palatable and varied food. However, despite the obvious growing interest in this hypothesis, it has not been tested empirically to the author's knowledge. It was one of the aims of the current dissertation to do so.

\section{Outline of the conducted studies and hypotheses}

The aim of the current dissertation is twofold. First, an attempt is made to demonstrate the causal link between impulsivity and overeating. Second, an attempt is made to demonstrate that the interaction effect of an impulsive personality and exposure to a toxic food environment on food intake is linked to increased food intake compared to the 
simple effects of impulsivity and the environment. Chapter 2 and 3 centre around the causal theme, whereas chapter 4 and 5 are concerned with the interaction between an impulsive personality and a toxic environment and its effect on overeating.

In chapter 2 an attempt was made to prime the concept of impulsivity in healthy participants. Female undergraduate students participated in two conditions. They did two priming tasks: one that hinted subtly at the concept of impulsivity and one that was neutral in content. Each time the priming task was followed by a bogus taste test. Trait impulsivity was measured by means of a behavioural measure, the stop signal task, and two self-report measures, the BIS and the $\mathrm{I}_{7}$. The main hypotheses were that participants would eat significantly more during the taste test after they had been primed with the concept 'impulsivity' compared to after the control session and that a more impulsive personality would predict a heightened food intake.

Two additional methods that could potentially induce impulsivity/inhibition were tested and their effect on food intake in the lab was determined in chapter 3 . In the first study healthy young women were cognitively primed with the concepts 'impulsivity' or 'inhibition'. In the second study impulsivity/inhibition was manipulated via behavioural instructions. In both studies the manipulation was followed by a bogus taste test. The main hypothesis was that inducing impulsivity would lead to a significantly higher food intake compared to inducing inhibition.

Chapter 4 describes a study in which it is investigated to what extent an impulsive personality, a varied food environment and their interaction influence food intake. A sample of 86 young healthy women completed the BIS, a self-report measure of impulsivity, and the stop signal task, a behavioural measure of impulsivity. Based on these measures the participants were labelled high or low impulsives. Under the guise of a taste test, both the high and low impulsive women were either served varied or monotonous food. Our main expectation was that high impulsives would eat significantly more during the taste test compared to the low impulsives.

Chapter 5 tested once more whether the interaction between a varied food environment and an impulsive person would lead to overeating. The link between impulsivity and overweight was also examined. The difference between this study and the study in chapter 3 is that the variety manipulation was made stronger (colour, form, taste, and texture were all varied) and that the sample consisted of 78 primary school children (age: $8-10 y$ ) instead of young women. We measured two aspects of impulsivity. Rewardsensitivity was measured using the door opening task and deficient response inhibition 
was measured using the stop signal task. Subsequently, one aspect of the obesogenic environment was manipulated: half of the participants received monotonous food during a bogus taste test whereas the other half tasted food that was varied in colour, form, taste and texture. It was expected that both deficient response inhibition and rewardsensitivity would interact with variety, resulting in a significantly higher food intake compared to the simple effects of an impulsive personality or a varied food environment. A positive association between overweight and both deficient response inhibition and reward-sensitivity was also hypothesized. 
Part I

Impulsivity as a cause of overeating 



\section{Chapter 2}

The influence of trait and induced state impulsivity on food intake in normal-weight healthy women

Published as: Guerrieri, R., Nederkoorn, C., Stankiewicz, K., Alberts, H., Martijn, C, \& Jansen, A. (2007 b). The influence of trait and induced state impulsivity on food intake in normal-weight women. Appetite, 49, 66-73. 


\begin{abstract}
Previous research has linked overeating and overweight/obesity to impulsivity. To find out whether impulsivity causes overeating and hence overweight and obesity, we attempted to prime the concept of impulsivity in healthy participants. In a within-subjects design one sample participated in two conditions. In both conditions participants did a priming task that either hinted subtly at the concept of impulsivity or that was neutral in content. Each time the priming task was followed by a bogus taste test. Trait impulsivity was measured by means of a behavioural task and self-report. Firstly, we hypothesized that participants would eat more during the taste test after they had been primed with the concept "impulsivity" compared to after the control session. Secondly, we expected that a more impulsive personality would predict a heightened food intake. Thirdly, we expected that impulsivity would predict food intake better than restraint. The results showed that both the self-report measure of impulsivity and the behavioural task predicted food intake. Restraint did not significantly predict food intake. Primed impulsivity did not increase food intake, possibly because the priming effect did not last long enough.
\end{abstract}

Keywords: Impulsiveness; Inhibitory control; Priming; Food intake 


\section{Introduction}

The World Health Organisation (WHO) has declared obesity a global epidemic (WHO, 2003). This epidemic brings with it a rise in chronic diseases like diabetes and cancer (Hu, 2003). Quality of life from a psychological and social point of view is in many cases also seriously affected (Karlsson, Taft, Sjöström, Torgerson, \& Sullivan, 2003). Besides the physical, psychological and social consequences for the individual, there are the financial consequences for society. In the United States an estimated 9\% of medical expenditures are attributable to overweight and obesity (Flegal, 2005). It is clear that something needs to be done to stop and even reverse this obesity epidemic. For this reason much research has been done on causal and maintaining factors of obesity. Genetic, biological, psychological and sociocultural factors have been suggested (Drewnowski, 1991).

One such psychological factor is impulsivity. Generally impulsivity is defined as the tendency to think, control and plan insufficiently. In most cases this results in an inaccurate or maladaptive response (Solanto et al., 2001). Impulsivity is considered a multidimensional construct (Wingrove \& Bond, 1997) for two reasons. First, correlations between self-report measures and behavioural measures of impulsivity are generally weak. This could mean that self-report impulsiveness questionnaires measure a different aspect of impulsivity compared to behavioural impulsivity tasks. Second, even within the behavioural tasks different operationalisations and explanatory models of impulsivity are used and these often intercorrelate poorly.

Roughly, one could say that there are three main aspects of impulsivity. The first is impulsiveness, defined as 'acting on the spur of the moment without being aware of any risk involved' (Eysenck, Easting, \& Pearson, 1984, pp. 315) and measured by selfreport. The second is response inhibition, which is predominantly measured by behavioural tasks. Barkley (1997) suggests that inhibitory control is necessary for executive functions such as self-regulation. If response inhibition is disturbed, then the very first self-regulatory act of inhibiting the prepotent response already fails, with all its consequences. The third aspect of impulsivity is sensitivity to reward, which is measured both by behavioural tasks and by self-report questionnaires. People who are sensitive to reward detect more rewarding stimuli and are more likely to approach these rewarding stimuli (Avila, 2001). It should be noted that impulsiveness and response inhibition, but not reward sensitivity, are the focus of the current study.

One can imagine that all three aspects of impulsivity contribute to the obesity epidemic (Davis, Levitan, Smith, Tweed, \& Curtis, 2006). First, when one often acts on 
the spur of the moment, momentary craving might be more important than future goals of losing weight. Second, when one is confronted with palatable food, the prepotent response is to eat it. In times when food was scarce this was an adaptive response (Blundell \& Gillett, 2001). However, it is feasible that in today's toxic environment not being able to inhibit one's prepotent responses contributes significantly to the problem of obesity. Third, sensitivity to reward might lead people make the wrong food choices: they might prefer foods that are sweet and fat because palatable food has a greater rewarding value than bland food (Davis, Patte, Levitan, Reid, Tweed, \& Curtis, 2007).

Research has begun to demonstrate a link between overeating and impulsivity, measured with self-report, with response inhibition tasks and with sensitivity to reward tasks. Nasser and colleagues (Nasser, Gluck, \& Geliebter, 2004) have shown that Binge Eating Disorder (BED) patients score significantly higher on a self-report measure of general impulsiveness compared to controls. They also found positive significant correlations ( .50) between participants' impulsivity score and the BED criteria "Loss of control during a binge" and "Eating when not physically hungry". Nederkoorn and colleagues found that obese children were impaired in general response inhibition as measured by the stop-signal paradigm compared to control participants (Nederkoorn, Braet, Van Eijs, Tanghe, \& Jansen, 2006). In other words, even on a very basic motoric level that has nothing to do with food the obese children were less able to inhibit their responses. Moreover, impulsivity turned out to be an obstacle in the treatment of the obese children: the children that were worst at inhibiting responses, lost less weight (Nederkoorn, Braet, et al., 2006; Nederkoorn, Jansen, Mulkens, \& Jansen, 2007)

Nederkoorn and colleagues (Nederkoorn et al, 2006) also found that the obese children were more sensitive to reward during a behavioural task compared to control children. Other research has shown that obese children have difficulties with delay of gratification tasks only when the incentive is edible (Bonato \& Boland, 1983). This suggests that food could be especially rewarding for the obese. This has indeed been found. In a study by Saelens and Epstein (1996) obese participants chose to work for food instead of sedentary activities more often than controls. In sum, overweight and obese people seem more sensitive to reward and less adequate at the inhibition of prepotent responses, especially when it comes to resisting palatable food.

Recently it has been shown that even in healthy, lean participants impulsivity is of importance when it comes to food. Healthy individuals who are more sensitive to reward according to a self-report questionnaire turned out to have more pronounced neural re- 
sponses to images of appetizing food (Beaver et al., 2006). This could indicate that for high-impulsive people it is harder to resist food than for low-impulsive people. Indeed, Guerrieri, Nederkoorn, and Jansen (2007a) found that high-impulsive women ate more during a bogus taste test than their low-impulsive peers when impulsivity was measured by self-report, but not by the stop-signal paradigm. The high-impulsive women also scored significantly higher on the Eating Disorders Examination Questionnaire (EDE-Q; Fairburn, \& Beglin, 1994). This indicates that the high-impulsives exhibited more "eating disordered" cognitions and behaviours compared to the low-impulsives, although the clinical cut-off scores for eating disorders were not reached.

In conclusion, impulsivity in all its forms has repeatedly been linked to overweight and obesity. Although there are exceptions (see Guerrieri et al., 2007a) impulsivity seems to be linked to reactions to palatable food, to "eating disordered" cognitions and to overeating in healthy participants. The relationship between increased impulsivity and the problem of overeating is quite robust since it generally persists even when impulsivity is measured in different ways (self-report versus behavioural tasks; response inhibition versus sensitivity to reward). However, based on previous research one cannot draw conclusions on causality. The studies that have been conducted are mostly of a correlational or quasi-experimental nature. This makes it impossible to rule out a third variable that influences impulsivity as well as overeating. If one wishes to conclude that impulsivity causes a heightened food intake, one needs to manipulate impulsivity experimentally. One should randomly assign healthy subjects to one of two groups: an experimental group in which impulsivity is manipulated experimentally versus a control group. If the experimental group shows a heightened food intake during a bogus taste test, then one could rightfully conclude that increased impulsivity caused the heightened food intake. We could find only one study in which similar methodology was used. Rotenberg and colleagues (2005) primed "lack of control" thoughts and these thoughts did indeed lead to greater food intake compared to priming "control" thoughts. This suggests that impulsivity, which shows overlap with the construct of "lack of control", might indeed promote overeating in a sample of healthy participants. However, no neutral condition was included, so it is not clear whether "control" cognitions led to less food intake, whether "lack of control" cognitions led to increased food intake, or both.

Because of the importance of this type of experimental research, which is the only way to investigate the causal relation between impulsivity and overeating, we tried to generalize the results of Rotenberg and colleagues (2005). We included a neutral control 
condition to avoid the issues in the Rotenberg study. We attempted to manipulate impulse control experimentally by means of a priming task that differed from the task that Rotenberg and colleagues (2005) used. Priming is a frequently used method in social cognition research. It is applied to enhance the cognitive availability of a psychological construct. Research has shown that priming procedures affect people's judgments and behaviour (Higgins, Rholes, \& Jones, 1977; Bargh, Gollwitzer, Lee-Chai, Barndollar, \& Trötschel, 2001). In the current study priming was used to manipulate the accessibility of the impulsivity construct in the hope that this priming would affect eating behaviour. In a within-subjects design one sample participated in two conditions. In both conditions participants did a priming task that either hinted subtly at the concept of impulsivity or that was neutral in content. After the priming task participants did a bogus taste test in both conditions. Trait impulsivity was measured by means of a behavioural task and selfreport.

We hypothesized that participants would eat more during the taste test after they had been primed with the concept "impulsivity" compared to after the control session. In light of the findings of Guerrieri and colleagues (2007a) it was also expected that a more impulsive personality would predict a heightened food intake. It is possible that trait impulsivity interacts with the priming task. In other words, high-impulsive people could react differently to the priming of impulsivity compared to low-impulsive people. However, we had no specific expectations concerning the presence of this interaction or its specific form.

Restraint (Herman \& Polivy, 1980) or the extent to which people diet (mostly without success) and worry about their weight, is another important variable to consider when looking at eating behaviour. It has been shown repeatedly that, compared to unrestrained eaters, restrained eaters consume more food after having consumed a highcaloric preload. This is called "counterregulation" (Herman \& Polivy, 1980). This counterregulation is thought to occur because the consumption of the preload acts as a disinhibitor: it removes inhibitions and eliminates restraint. It turned out that it is not even necessary to consume food in order to disinhibit restrained eaters. Mere exposure to food stimuli is sufficient to break down dietary restraint (Jansen \& van den Hout, 1991; Rogers and Hill, 1989). In other studies participants who were identified as restrained eaters were found to eat more than unrestrained eaters when they were given ad libitum access to palatable food, not preceded by a preload or cue exposure (e.g., Jansen, 1996). Moreover, Nederkoorn, Van Eijs and Jansen (2004) found that restrained eaters were worse at 
prepotent response inhibition, i. e. more impulsive, compared to controls. In combining these findings we can conclude that the heightened food intake in restrained eaters may be a consequence of their increased impulsivity. Hence, we hypothesized that in the current study impulsivity would predict food intake better than restraint.

In sum, we had three hypotheses: (a) Participants will eat more during the taste test after they have been primed with the concept "impulsivity" compared to after the control session (b) a more impulsive personality will predict a heightened food intake (c) impulsivity will predict food intake better than restraint. Moreover, we explored whether trait impulsivity would interact with the priming of impulsivity.

\section{Method}

Participants

Forty-two normal-weight female students were recruited to participate in a study on "taste perception". Four participants were excluded because they missed one or more sessions. This left us with thirty-eight participants (mean age: $19.3 \pm 1.3$ years; mean BMI: $22.2 \pm 2.9$ ). They received course credit or a monetary reward for their participation. All participants were debriefed by e-mail.

\section{Materials}

The Scrambled Sentences Task (Srull \& Wyer, 1979) is a priming method that originates from social psychology. To our knowledge, this priming task has never been used to prime impulsivity, but is has already been used successfully to prime control (Araya, Akrami, Ekehammar, \& Hedlund, 2002). It is believed that a schema associated with a certain trait will be activated when one is exposed to behavioural examples of this trait. Unobtrusive exposure to behavioural examples of a trait is attained as follows. Participants receive five words in a randomized order. It is their task to construct a grammatically correct sentence with four out of five given words. This task exposes the participants to the intended content while it averts their attention from the contents to the syntax of the sentences (Kühnen \& Hannover, 2000). In the current study participants constructed 25 sentences per session. They were only required to say the unscrambled sentences out loud. This saved considerable time compared to letting participants write down the unscrambled sentences. During the experimental session fifteen sentences hinted subtly at the concept of impulsivity (e.g. Patrick lets himself go) and ten sentences served as fillers (e.g. Donald reads the paper). During the control session participants 
constructed 25 sentences that were neutral in content. It should be noted that sentences that had anything to do with food were avoided.

The Barratt Impulsiveness Scale (BIS; Patton et al., 1995) is a self-report questionnaire that measures trait impulsiveness. It consists of 30 items to be rated on a 4-point scale. Scores range from 30 to 120 with higher scores indicating more impulsiveness. The BIS consists of three subscales: motor impulsiveness (acting without thinking), attentional impulsiveness (not focusing on the task at hand, cognitive instability) and nonplanning impulsiveness (lack of orientation to the future).

The $I_{7}$ Impulsiveness Questionnaire ( $I_{7}$; Eysenck et al., 1985) is a 54-item self-report questionnaire that aims to measure trait impulsiveness, venturesomeness and empathy. Scores range from 0 to 54 with higher scores indicating more impulsiveness.

The Stop Signal Paradigm (Logan et al., 1997) is based on the notion that impulsive behaviour can be operationalised as a diminished ability to inhibit prepotent responses. In order to measure inhibitory control a behavioural computer task was developed (Logan et al., 1997). This computer task contains two sorts of trials: go trials (75\%) and stop trials (25\%). During the go trials the participant performs a choice reaction time task: the participant learns to press a certain button as fast as possible dependent on the stimulus that is presented (an X on the right or an $\mathrm{O}$ on the left for $1500 \mathrm{~ms}$ ). This learned response has to be inhibited during the stop trials: a tone serves as a stop signal and tells the participant not to push the button in response to the stimulus. At the start of the task the delay between the go signal ( $\mathrm{X}$ or $\mathrm{O}$ ) and the stop signal is set to $250 \mathrm{~ms}$. A tracking procedure adapts the delay dynamically depending on the participant's behaviour. If the participant inhibits successfully, the task is made more difficult by increasing the delay by $50 \mathrm{~ms}$. In the case of an unsuccessful inhibition the delay decreased by $50 \mathrm{~ms}$, making the task easier. The task consists of four blocks of 64 trials each and a practice block of ten trials. Two variables are measured: reaction time (RT) and stop delay. The stop signal reaction time (SSRT), the main independent variable, is calculated by subtracting the stop delay from the reaction time (Logan et al., 1997). The longer the SSRT, the more impulsive a participant is thought to be.

The Restraint Scale (RS; Herman \& Polivy, 1980) collects information on attitudes towards weight, degree and frequency of dieting, loss of control over eating and weight fluctuations. Scores range from 0 to 40 .

In order to measure food intake, participants took part in three bogus taste tests using three milkshakes ( $260 \mathrm{~g} / 420 \mathrm{kcal}$ per milkshake) per taste test. The milkshakes 
were vanilla flavour during the first session and strawberry flavour during the second and the third session. About ten minutes before the arrival of the participant we prepared the milkshakes by mixing $100 \mathrm{ml}$ of whole milk with $150 \mathrm{~g}$ of ice cream with whipped cream in a milkshake machine. Strawberry flavour was added to the milkshakes that were used during the second and third session. The participants were asked to taste three milkshakes and to fill in a Taste Test Questionnaire. Participants had to rate the milkshakes on creaminess, sweetness and overall palatability. They also had to fill in a number of open-ended questions on the taste and mouth feel of the milkshakes. Moreover, the participants had to indicate which of the three milkshakes had a slightly different taste compared to the other two. This was all done to give the participants a chance to consume the milkshakes. The data from this questionnaire were not analysed, except for three items that measured how much participants felt like tasting the shakes (ranging from 1: "not at all" to 5: "very much") and how strong their intentions were to drink the milkshakes and to buy the milkshakes (ranging from 1: "not strong at all" to 5: "very strong").

Mood and bunger measurements were taken in order to rule out pre-existing differences in hunger and mood between the neutral and experimental session. At the beginning of each session hunger and mood was measured on a five-point scale that ranged from "not at all" (1) to "very" (5). The moods that were measured were: happy, sad, anxious, nervous, relaxed, angry, disappointed, and energetic.

In order to rule out participants who knew that we had tried to prime impulsivity or who were on to the true hypotheses of the study, a suspiciousness questionnaire was devised. Participants were asked to try to come up with a general theme for the sentences that they had constructed. Furthermore, participants had the opportunity to write down what they thought the true hypotheses of the study were. None of the participants named "impulsivity" or a linked theme and likewise none of the participants came close to the true hypotheses of the study.

\section{Procedure}

Participants had to complete four testing sessions: three individual sessions and one group session. For each participant the three individual sessions were planned on three consecutive days at approximately the same time of day. The group sessions were planned every three to four weeks. 
The first individual session lasted approximately 40 minutes. Participants started with the stop-signal task, followed by a taste test using three vanilla milkshakes. This taste test was done in order to give participants a chance to get used to eating in a laboratory situation. We knew from previous experience that participants exhibit a "neophobia effect" (Overduin \& Jansen, 1997; Roefs \& Jansen, 2004). This means that they eat far less when they have to eat in a lab for the first time compared to subsequent occasions. We did this first taste test to solve this problem.

During the second individual session, which lasted approximately 30 minutes, participants filled in a general questionnaire that measured hunger and mood. This was followed by the Scrambled Sentences Task. Participants started with the experimental sentence task or the control sentence task, in a balanced order. After the sentence task participants did a taste test that was the same as the first one except for the use of strawberry milkshakes instead of vanilla milkshakes.

The third individual session was the same as the second. Participants who did the experimental sentence task during the second session, did the control sentence task during this session and vice versa. The same flavour (strawberry) was used during the taste test. After filling in the suspiciousness questionnaire, participants were weighed and measured.

The last session, the group session, served to let the participants fill in the questionnaires that measured impulsivity and restraint: the $I_{7}$, the BIS and the RS. It lasted approximately 20 minutes.

\section{Results}

Pre-existing differences and neophobia effect

Paired-samples t-tests indicated that there were no pre-existing differences in hunger and mood between the neutral and the experimental session (see Table 2.1). We did find a neophobia effect: a within-subjects ANOVA indicated that at least one session differed from the remaining sessions as far as food intake was concerned, $F(2,74)=8.28, p<0.01$. Post-hoc analyses with Bonferroni correction ( $\alpha$ divided by 3: $\alpha=0.017$ ) indicated that the food intake during session 1 was significantly lower compared to session $2, t(37)=$ $2.82, p<0.017$ and $3, t(37)=3.55, p<0.017$. The difference between session 2 and 3 was not significant, $t(37)=1.65, p>0.1$. The mean food intake was $119 \mathrm{~g}$ (SD $74 \mathrm{~g}$ ) for session $1,152 \mathrm{~g}$ (SD $94 \mathrm{~g}$ ) for session 2 and $174 \mathrm{~g}$ (SD $114 \mathrm{~g}$ ) for session 3. 
Hypothesis 1: Participants will eat more during the taste test after they have been primed with the concept "impulsivity" compared to after the control session

A paired-samples t-test indicated that food intake during the experimental and control condition did not differ significantly. Intention to drink and buy the milkshakes also did not differ significantly. However, during the experimental session participants felt more like tasting the milkshakes compared to the control session (see Table 2.2 for descriptives, test statistics and significance levels). Order effects were tested and were not significant, $F(1,36)=2.7, p>0.1$.

\section{Table 2.1}

Hunger and mood measurements at the beginning of the neutral and experimental session, measured on a five-point scale ranging from "not al all" (1) to "very" (5)

\begin{tabular}{|c|c|c|c|c|c|c|}
\hline & \multicolumn{2}{|c|}{ Neutral Session } & \multicolumn{2}{|c|}{ Experimental Session } & \multicolumn{2}{|c|}{ for difference N-E } \\
\hline & M & SD & M & SD & $t(37)$ & $p$ \\
\hline hungry & 2.95 & 1.14 & 3.00 & 1.04 & -0.24 & 0.81 \\
\hline happy & 3.66 & 0.82 & 3.82 & 0.69 & -1.18 & 0.24 \\
\hline sad & 1.39 & 0.79 & 1.29 & 0.73 & 0.94 & 0.35 \\
\hline anxious & 1.21 & 0.57 & 1.08 & 0.27 & 1.40 & 0.17 \\
\hline nervous & 1.34 & 0.78 & 1.47 & 0.76 & -1.09 & 0.28 \\
\hline relaxed & 3.66 & 0.94 & 3.68 & 0.93 & -0.14 & 0.89 \\
\hline angry & 1.26 & 0.69 & 1.11 & 0.31 & 1.64 & 0.11 \\
\hline disappointed & 1.26 & 0.72 & 1.18 & 0.39 & 0.68 & 0.50 \\
\hline energetic & 3.18 & 0.93 & 3.24 & 0.88 & -0.32 & 0.75 \\
\hline
\end{tabular}

Note. $\mathrm{N}-\mathrm{E}=$ mood scores obtained during the Experimental Session were subtracted from the mood scores obtained during the Neutral Session 


\section{Table 2.2}

Differences between the neutral versus the experimental session for food intake, and 3 taste test questions, measured on a five-point scale ranging from "not strong at all" (1) to "very strongly" (5)

\begin{tabular}{|c|c|c|c|c|c|c|}
\hline & \multicolumn{2}{|c|}{ Neutral Session } & \multicolumn{2}{|c|}{ Experimental Session } & \multicolumn{2}{|c|}{ for difference N-E } \\
\hline & M & SD & M & SD & $t(37)$ & $p$ \\
\hline Food intake (g) & 167 & 115 & 158 & 95 & 0.63 & 0.54 \\
\hline $\begin{array}{l}\text { How much do you feel } \\
\text { like tasting the milk- } \\
\text { shakes? }\end{array}$ & 2.74 & 1.37 & 3.18 & 1.06 & -2.01 & 0.05 \\
\hline $\begin{array}{l}\text { Intention to drink these } \\
\text { milkshakes }\end{array}$ & 2.43 & 1.26 & 2.51 & 1.33 & -0.68 & 0.50 \\
\hline $\begin{array}{l}\text { Intention to buy these } \\
\text { milkshakes }\end{array}$ & 1.55 & 1.39 & 1.50 & 1.35 & 0.40 & 0.69 \\
\hline
\end{tabular}

Note. $\mathrm{N}-\mathrm{E}=$ mood scores obtained during the Experimental Session were subtracted from the mood scores obtained during the Neutral Session

Hypotheses 2 and 3: A more impulsive personality will predict a heightened food intake and impulsivity will predict food intake better than restraint

Because BIS and $\mathrm{I}_{7}$ scores correlated significantly, $r=0.41, p<0.05$, both scores were standardized and then averaged to result in one self-report score for impulsiveness (Self-Report Impulsiveness). The Stop Signal Reaction Time (SSRT) served as the behavioural measure of impulsivity (Behavioural Impulsivity). These measures were included in a correlation matrix (see Table 2.3), together with Total Food Intake and the Restraint Scale (RS). Only the correlation between Behavioural Impulsivity and Total Food Intake was significant. The correlation between Behavioural Impulsivity and Self-report Impulsiveness was not significant, so both measures could be included as predictors in the linear regression analysis.

Both impulsivity measures and the RS scores were centred and used as predictors in a multivariate linear regression analysis with Total Food Intake (sum of session 1, 2 and 3) as the dependent variable. This model was significant, $r^{2}=0.25, F(3,34)=3.7, p<$ 0.05 . Behavioural Impulsivity proved to be a significant predictor of Total Food Intake, $\beta$ $=0.33, t(37)=2.15, p<0.05$, and Self-report Impulsiveness was a marginally significant 
predictor of food intake, $\beta=0.29, t(37)=1.91, p<0.1$. However, RS did not predict Total Food Intake, $\beta=0.21, t(37)=1.35, p>0.1$.

Removing the nonsignificant predictor led to a more significant model, $r^{2}=0.21$, $F(2,35)=4.54, p<0.02$, in which both Self-report Impulsiveness, $\beta=0.32, t(37)=2.08, p<$ 0.05 , and Behavioural Impulsivity, $\beta=0.38, t(37)=2.46, p<0.02$, proved to be significant predictors of total food intake.

Table 2.3

Pearson product-moment correlations between Total Food Intake, the self-report measurement of impulsiveness (Self-report Impulsiveness), the behavioural measurement of impulsivity (Behavioural Impulsivity), and the Restraint Scale (RS).

\begin{tabular}{lllll}
\hline & $\begin{array}{l}\text { Total } \\
\text { Intake }\end{array}$ & $\begin{array}{l}\text { Food } \\
\text { siveness }\end{array}$ & $\begin{array}{l}\text { Self-report Impul- } \\
\text { pehavioural } \\
\text { pulsivity }\end{array}$ & Im- \\
\hline Self-report Impulsiveness & 0.26 & - & \\
Behavioural Impulsivity & $0.33^{*}$ & -0.14 & - \\
RS & 0.30 & 0.10 & 0.20 & \\
\hline
\end{tabular}

${ }^{*}$ Correlation is significant at the 0.05 level (two-tailed)

\section{Exploration: Interaction between State and Trait Impulsivity}

A 2 (neutral versus experimental condition; WS) by 2 (low versus high impulsives; BS) mixed model ANOVA showed that there was no significant interaction between state and trait impulsivity both when trait impulsivity was measured by the stop-signal task, $F(1,36)=2.40 ; p>0.1$, and by self-report, $F(1,36)=0.25 ; p>0.6$. See Table 2.4 for descriptives. 
Table 2.4

Mean food intakes $(\mathrm{g})$ and standard errors for interaction effects between trait and state impulsivity State impulsivity $\quad \mathrm{M} \quad \mathrm{SE}$

Trait Impulsivity:

Self-Report

Low-impulsives Neutral Priming Task $\quad 146 \quad 25$

Impulsive Priming Task $131 \quad 19$

High-impulsives Neutral Priming Task $\quad 201 \quad 28$

Impulsive PrimingTask $\quad 192 \quad 21$

Trait Impulsivity: State Impulsivity

$\underline{\text { Behavioural }}$

$\begin{array}{llcc}\text { Low-impulsives } & \text { Neutral Priming Task } & 135 & 26 \\ & \text { Impulsive Priming Task } & 141 & 21 \\ \text { High-impulsives } & \text { Neutral Priming Task } & 188 & 26 \\ & \text { Impulsive Priming Task } & 169 & 21\end{array}$

\section{Discussion}

In accordance with our previous study (Guerrieri et al., 2007a) we found that trait impulsivity predicts heightened food intake in normal-weight healthy women. This was the case for both measures of impulsivity: self-report and behavioural. The low correlation between both sorts of measures has been found in other studies (Wingrove \& Bond, 1997) and it might indicate that self-report and behavioural measures of impulsivity measure different aspects of impulsivity in motor, cognitive, social and emotional domains (Solanto et al., 2001). Another reason for the low correlation might lie in the qualitative differences between self-report and behavioural measures (Enticott, Ogloff, \& Bradshaw, 2006). On the one hand, self-report measures are sensitive to a person's biases and inaccuracies whereas behavioural measures are considered to be more objective. On the other hand, the environment in which laboratory tasks are administered might be too neutral whereas the items of self-report measures usually refer to real-life situations. 
In the current study both measures of impulsivity predict food intake. This result supports the possibility that impulsive people overeat more easily. Because of this they might be more prone to develop overweight or even obesity. This might also explain why overweight and obese people have been found to be more impulsive and why obesity has been linked to $\mathrm{AD} / \mathrm{HD}$, a disorder that is hallmarked by an excess of impulsive behaviour (Davis et al., 2006). Disproportionate amounts of children with AD/HD were found within a group of children who were hospitalized for severe obesity (AgranatMeged et al., 2005). The reverse also turned out to be true: the mean BMI in a sample of $\mathrm{AD} / \mathrm{HD}$ boys was significantly higher than the age-adapted reference values (Holtkamp et al., 2004).

As hypothesized, impulsivity scores predicted food intake better than Restraint. The nonsignificant correlation between RS and impulsivity and RS and food intake does not support the theory that impulsivity mediates the effect of Restraint on food intake. It is possible that this is only the case when Restraint scores are extreme. Nederkoorn and colleagues (2004), who found that highly restrained participants were worse at prepotent response inhibition, i. e. more impulsive, selected their participants on the basis of RS scores $(<6$ and $>15)$. This resulted in more extreme groups. With a mean of 11.5 and a standard deviation of 4.8 Restraint scores were not at all extreme in the current sample.

Despite an effect of trait impulsivity on food intake we were not able to demonstrate that experimentally manipulated impulsivity affects the participants' food intake. In other words, we could not demonstrate that impulsivity causes heightened food intake. There are two possible reasons for not finding this effect. First, it is possible that the priming worked, but that state impulsivity does not influence food intake. Second, it can be that impulsivity does influence food intake, but that our manipulation was not strong enough, or did not last long enough to elicit an effect on food intake. In this case the latter option seems more likely: participants felt more like tasting the milkshakes immediately after the impulsive priming task compared to the control priming task. However, the intention to drink and to buy the milkshakes, items that were in the middle and at the end of the taste test questionnaire did not show this difference between the two sessions. This could indicate that there was an effect of priming, but that it only lasted for a very short period of time. In the study of Rotenberg and colleagues (2005) the priming task was presented as a memory task. The benefit is that participants had to keep the priming words in mind during the taste test. This enhances the probability of successful priming. In the current study the sentences that were constructed during the Scrambled Sen- 
tences Task did not have to be remembered until after the taste test. This might explain why the priming effect in this study was not strong enough to affect food intake. In future studies it might be wise to ensure that participants need to keep the priming words in mind until after the taste test. It has also been suggested that negative affect is needed for impulsive behaviour to occur (Fischer, Smith, \& Anderson, 2003). If this is the case, future priming studies should not only aim to induce impulsivity, but also negative affect.

Ideally one would use a state measure of impulsivity as a manipulation check when trying to induce impulsivity. If participants score higher on this measure in the experimental condition compared to the control condition, then one is sure that the manipulation led to more food intake via increased impulsivity. However, using this kind of manipulation check was very difficult in the current study for two reasons. First, to our knowledge, a validated state measure of impulsivity does not exist. Second, the timing of the manipulation check is very difficult to determine. If one does the manipulation check immediately after the manipulation, one risks that the priming effect is already weakened once participants get to the taste test. If one does the check after the taste test, chances are that the manipulation did work during the taste test, but that the priming effect is too weak by the time participants get to the manipulation check. The timing problem could be resolved by conducting two studies: one study in which the manipulation is validated and a second study in which the effect of the manipulation on food intake is measured. However, the need for a validated state measure of impulsivity remains.

When inducing a state in order to establish a causal relationship between a trait and certain behaviors it is an issue whether the state that is induced is qualitatively comparable to the trait that it is supposed to represent. In other words, is the effect of being put in an "impulsive mood" comparable to the effect of having an impulsive personality? In the area of impulsivity little attention has been paid to this issue. More work has been done in the area of anxiety. Although trait and state anxiety are measured separately (Van der Ploeg, Defares, \& Spielberger, 1980), they are considered to be closely linked. The genes that influence state and trait anxiety are identical and people with high levels of trait anxiety are more likely to become state-anxious in reaction to a situation that is seen as threatening (Lau, Eley, \& Stevenson, 2006). If this reasoning also applies to impulsivity one could say that people with an impulsive personality are simply more prone to be in an impulsive mood. If it is indeed so that the effect of a trait on behaviour is mediated by its state, then it should not matter whether this state is induced or occurring naturally. However, further research is needed before this issue can be resolved. 
Lastly, a methodological concern was that neophobia would occur. This means that participants need to get used to eating in the lab and consequently they eat far less the first time compared to a second and a third time. This is indeed what we found. If one has two experimental sessions this effect is not wanted because it would interfere with the experimental situation. That is why it is recommended that one lets participants eat in the lab once to let them get used to eating in this unnatural environment before one starts with experimental sessions.

In summary, the hypothesis that increased impulsivity causes a heightened food intake was not supported. The priming task that was used probably did not elicit a priming effect that was strong enough to affect food intake during the subsequent taste test. However, this study does show that in healthy, lean participants impulsivity, measured by both self-report and behavioural tasks, predicts food intake during a bogus taste test in the lab. In other words, the connection between impulsivity and food intake was supported. Further studies should concentrate on the induction of impulsivity in healthy subjects. This is the only way to establish whether increased impulsivity indeed causes a heightened food intake. 



\section{Chapter 3}

Inducing impulsivity leads restrained eaters into overeating, whereas current dieters stick to their diet

In revision as: Guerrieri, R., Nederkoorn, C., Schrooten, M., Martijn, C., \& Jansen, A. Inducing impulsivity leads restrained eaters into overeating, whereas current dieters stick to their diet 


\begin{abstract}
Previous research has related impulsivity to overeating and obesity. However, the precise nature of this relation has not been examined yet. One possibility is that impulsivity causes overeating and hence contributes to overweight. To test this possibility we induced impulsivity versus inhibition to see whether this would affect food intake. In the first study participants were cognitively primed with the concepts "impulsivity" or "inhibition". Caloric intake was significantly higher in the Impulsivity Condition compared to the Inhibition Condition. This effect was even stronger for highly restrained participants. In the second study impulsivity was manipulated via behavioural instructions. Restrained and unrestrained non-dieters acted as expected: their caloric intake was significantly higher when impulsivity was induced compared to inhibition. Current dieters sharply reduced their caloric intake following the impulsivity induction. These results are in accordance with Lowe's model that, contrary to restraint theory, states that restraint and current dieting are different constructs that affect eating regulation differently. At least for non-dieters it can be concluded that heightened impulsivity versus inhibition leads to a higher food intake in the lab.
\end{abstract}

Keywords: Impulsivity; Response Inhibition; Stop Signal Task; Obesity; Priming 


\section{Introduction}

Impulsivity is a personality trait that has been found a causal and/or maintaining factor in numerous psychopathologies and behavioural disorders, ranging from addiction (Moeller \& Dougherty, 2002) to AD/HD (Solanto et al., 2001). In general, impulsivity is defined as the tendency to think, control and plan insufficiently. In most cases this results in an inaccurate or maladaptive response (Solanto et al., 2001). Impulsivity is thought to be a multidimensional concept, so precise behaviours that are considered impulsive are very diverse. Roughly, one can distinguish three sorts of models: behavioural models, motivational models and personality models. The most widely cited behavioural model is that of Logan, Schachar, and Tannock (1997). In this model, impulsivity is seen as the diminished ability or inability to inhibit a prepotent or predominant response. In motivational models the emphasis is on reward. Impulsivity is operationalised as rewardsensitivity (e.g. Avila, 2001) or delay aversion (e.g. Metcalfe \& Mischel, 1991). Rewardsensitive people detect more rewarding stimuli and are more likely to approach these rewarding stimuli (Avila, 2001). Delay aversion or delay of gratification reflects the preference for a smaller sooner reward over a larger later reward (Solanto et al., 2001). The aim of personality models (e.g. Eysenck, Pearson, Eating, \& Allsop, 1985; Whiteside \& Lynam, 2001; Patton, Stanford, \& Barratt, 1995) is to clarify the construct of impulsivity, for example by linking it to major personality systems.

Recently, the quest for causal and maintaining factors of obesity has uncovered a link between these aspects of impulsivity, overeating and obesity. Nasser and colleagues (Nasser, Gluck, \& Geliebter, 2004) have shown that Binge Eating Disorder (BED) patients score significantly higher on a self-report measure of general impulsiveness compared to controls. They also found positive significant correlations ( .50$)$ between participants' impulsivity score and the BED criteria "Loss of control during a binge" and "Eating when not physically hungry". Nederkoorn and colleagues found that obese children were impaired in general response inhibition as measured by the stop-signal paradigm compared to control participants (Nederkoorn, Braet, Van Eijs, Tanghe, \& Jansen, 2006). In other words, even on a very basic motoric level that has nothing to do with food the obese children were less able to inhibit their responses. Moreover, impulsivity turned out to be an obstacle in the treatment of the obese children: the children that were worst at inhibiting responses, lost less weight (Nederkoorn et al., 2006; Nederkoorn, Jansen, Mulkens, \& Jansen, 2007). Nederkoorn and colleagues (Nederkoorn et al, 2006) also found that the 
obese children were more sensitive to reward during a behavioural task compared to control children.

It has been shown that even in healthy, lean participants impulsivity is of importance when it comes to food. Healthy individuals who are more sensitive to reward according to a self-report questionnaire turned out to have more pronounced neural responses to images of appetizing food (Beaver et al., 2006). This could indicate that for high-impulsive people it is harder to resist food than for low-impulsive people. Indeed, Guerrieri, Nederkoorn and Jansen (2007a) found that high-impulsive, but normal-weight, healthy women ate significantly more in the lab compared to low-impulsive women. Guerrieri, Nederkoorn, Stankiewicz, Alberts, Geschwind, Martijn et al. (2007b) demonstrated that impulsivity, measured by both a behavioural task and self-report, predicted a heightened cumulated food intake of three taste tests, spread over three days.

What these and most other studies on this topic have in common is that variables in the participants are observed in order to look for relationships between these variables. These kinds of studies cannot help us establish whether there exists a causal link between impulsivity, overeating and obesity.

In order to investigate whether impulsivity causes overeating, one needs to experimentally induce impulsivity and see whether this manipulation leads to a higher food intake compared to a control group. Something similar has been done by Rotenberg and colleagues (2005). They primed "lack of control" thoughts and these thoughts did indeed lead to greater food intake compared to priming "control" thoughts. Lack of control might be different from impulsivity in the sense that it puts more emphasis on the cognition of powerlessness, whereas impulsivity might be more related to behavioural activating. However, the concepts do show a great overlap and the results of the study do support the view that induced impulsivity might cause overeating, compared to induced control. The study of Guerrieri et al. (2007b) also attempted to prime the concept of impulsivity in order to affect food intake in the lab. Although the priming group did report higher craving for the food, no differences in food intake between the priming group and a control group were found and it was concluded that the manipulation might not have lasted long enough. Thus, although there are indications that impulsivity might be a cause of overeating, solid proof is still lacking.

We present two studies. The aim of these studies was mainly to influence food intake by inducing impulsivity, both cognitively and behaviourally. This would empirically support the possibility that impulsivity is causally linked to overeating. In the first study 
we attempted to cognitively prime the concepts of impulsivity and inhibition. Moreover, Restraint (Herman \& Polivy, 1980) was measured as a potential moderating factor. In the second study behavioural instructions were used to experimentally manipulate the participants into impulsive or inhibitory behaviour. Both Restraint (Herman \& Polivy, 1980) and Current Dieting (Lowe, 1993) were measured as potential moderating factors.

\section{Study 1}

This study had two aims. First, we wanted to demonstrate that impulsivity is causally linked to overeating. For this purpose we used a cognitive priming task. Priming is a frequently used method in social cognition research. It is applied to enhance the cognitive availability of a psychological construct. Research has shown that priming procedures affect people's judgments and behaviour (Higgins, Rholes, \& Jones, 1977; Bargh, Gollwitzer, Lee-Chai, Barndollar, \& Trötschel, 2001). In the current study priming was used to manipulate the accessibility of the "impulsivity" construct for half of the participants (Impulsivity Condition) and the "inhibition" construct for the rest of the participants (Inhibition Condition). After this priming task participants did a bogus taste test. It was hypothesized that participants in the Impulsivity Condition would have a significantly higher caloric intake compared to the participants in the Inhibition Condition.

Second, it was hypothesized that the effect of impulsivity on overeating would be moderated by Restraint (Herman \& Polivy, 1980). Restrained eaters, identified by the Restraint Scale, are believed to be (chronic) dieters or weight suppressors (Herman \& Polivy, 1980). It has been shown repeatedly that, compared to unrestrained eaters, restrained eaters consume more food after having consumed a high-caloric preload. This is called "counterregulation" (Herman \& Polivy, 1980). This counterregulation is thought to occur because the consumption of the preload acts as a disinhibitor: it removes inhibitions and eliminates restraint. It turned out that it is not even necessary to consume food in order to disinhibit restrained eaters. Mere exposure to food stimuli is sufficient to break down dietary restraint (Jansen \& van den Hout, 1991; Rogers and Hill, 1989). In other studies participants who were identified as restrained eaters were found to eat more than unrestrained eaters when they were given ad libitum access to palatable food, not preceded by a preload or cue exposure (e.g. Jansen, 1996).

Since the priming of impulsivity should bring participants in an impulsive, disinhibited state, it should have the same effect on eating regulation as a classic disinhibitor: a preload. Consequently, we expect an interaction between participants' Restraint status 
and the condition to which they are assigned (Impulsivity versus Inhibition). The restrained eaters in the Impulsivity Condition are expected to be extra vulnerable to overeating.

In summary, we had two hypotheses (1) the participants primed with "impulsivity" will consume more calories during a bogus taste test than the participants that were primed with "inhibition" (2) the effect of impulsivity on overeating will be moderated by restraint status, meaning that in particular the restrained participants in the Impulsivity Condition will overeat.

\section{Method}

Participants

Fourty-six female undergraduate students were recruited to participate in a study on "memory and taste perception" (mean age $20.4 \pm 2$; mean BMI $21.99 \pm 2.44$ ). All participants were tested individually and received course credit or a monetary reward of $€$ 7.50 for their participation. The procedure of the study was approved by the Ethical Committee of Maastricht University

\section{Materials}

The Priming Task was presented to the participants in the form of a memory task. Both groups of participants read a story about New Year's Resolutions. The participants were told that they would have to answer questions about the story at the end of the experiment. They were also told that it would help their memory if they imagined that they were the main character in the story. The Inhibition Condition received a story in which Andrea talks about her New Year's resolutions: she will start studying for her exams in time and attend all lectures and she will save money regularly so that she will be able to go on that trip to Italy next summer. The Impulsivity Condition received a story in which Andrea says that one should not think too much about the future. Flexibility and spontaneity are more important. Andrea's only New Year's resolution is to enjoy life. In both stories, food, eating or drinking were not mentioned. The themes in the stories are believed to reflect impulsivity because they were based on items of two widely used impulsivity questionnaires: the Barratt Impulsiveness Scale (BIS; Patton et al., 1995) and Eysenck's $\mathrm{I}_{7}$ (Eysenck et al., 1985). The items used for the inhibition story were: "I am restless at plays or lectures" (BIS item 28; item was inverted), "I save regularly" (BIS item 10), "I plan trips well ahead of time" (BIS item 7). The items used for the impulsivity story 
were: "I am more interested in the present than in the future" (BIS item 27), "I act on the spur of the moment" (BIS item 20), "An evening out is more successful if it is unplanned or arranged at the last moment" ( $\mathrm{I}_{7}$ item 38$)$.

A Bogus Taste Test was used in order to measure food intake of the participants in an unobtrusive way. Participants were left alone for 15 minutes to taste and rate four sorts of food that were placed in front of them in large bowls: chocolate ( $\pm 1100 \mathrm{~g}$ ), wine gums ( $\pm 1500 \mathrm{~g})$, marshmallows $( \pm 1000 \mathrm{~g}$ ) and a savoury nut mix ( $\pm 1100 \mathrm{~g})$. Participants had to rate odour, general palatability and, depending on the sort of food, sweetness, creaminess, saltiness and crunchiness. However, the primary interest of the authors was not how participants rated the taste of the food, but how much they ingested of the food that was offered. Without the participants' knowledge the bowls of food were weighed before and after the taste test in order to establish food intake. The amounts eaten of each food were converted to calories. The sum of these calories was the dependent variable: total caloric intake.

The Restraint Scale (RS; Herman \& Polivy, 1980) collects information on attitudes towards weight, degree and frequency of dieting, loss of control over eating and weight fluctuations. Scores range from 0 to 40 . The higher the RS score, the more restrained a person is believed to be.

\section{Procedure}

At recruitment participants were asked to eat something small like a cheese sandwich two hours before the start of the experiment and to otherwise refrain from eating and drinking (except water). This was done in order to control for differing states of hunger between the conditions at the start of the experiment.

At their arrival in the lab, participants started with the "memory task", which was actually the priming task. Reading the story took about one minute. Afterwards participants did the taste test. After the taste test the participants had to answer some questions on the priming story in order to confirm the cover story. Participants reported their height and weight, and they filled out the Restraint Scale. It was also checked whether participants had any relevant knowledge concerning the precise aims of the study. Afterwards, the participants were thanked and dismissed. 


\section{Results}

Pre-existing differences

Independent-samples t-tests indicated that the Impulsivity Condition and the Inhibition Condition did not differ significantly in age, $t(44)=-0.98, p>0.3$, and BMI, $t(44)=$ $1.63, p>0.10$. See Table 3.1 for descriptives.

Likewise age, $t(44)=-0.86, p \geq 0.4$, did not differ between high and low-restrained participants, but BMI, $t(44)=2.43, p<0.05$, did. See Table 3.2 for descriptives.

\section{Table 3.1}

Means and standard deviations for relevant variables in Inhibition vs. Impulsivity Condition in Study 1

\begin{tabular}{|c|c|c|c|c|c|}
\hline & \multicolumn{2}{|c|}{ Inhibition } & \multicolumn{2}{|c|}{ Impulsivity } & \multirow[b]{2}{*}{$p$} \\
\hline & $\mathrm{M}$ & SD & $M$ & SD & \\
\hline BMI & 21.38 & 1.86 & 22.54 & 2.80 & 0.33 \\
\hline Age & 20.10 & 2.00 & 20.70 & 2.30 & 0.11 \\
\hline
\end{tabular}

\section{Table 3.2}

Means and standard deviations for relevant variables for low restrained (Low RS) vs. high restrained (High RS) participants in Study 1

\begin{tabular}{llllll}
\hline & Low RS & \multicolumn{4}{l}{ High RS } \\
\hline & M & SD & M & SD & $p$ \\
\hline BMI & 21.13 & 1.87 & 22.76 & 2.66 & 0.02 \\
Age & 20.14 & 1.46 & 20.67 & 2.62 & 0.40 \\
\hline
\end{tabular}

\section{Effect of priming and restraint on caloric intake}

The participants were divided into a restrained and an unrestrained group via a median split. The participants that scored below the median RS score 11 (a normal median for a Dutch sample) were considered to be unrestrained $(n=22)$ whereas participants with an RS score above $11(\mathrm{n}=24)$ were considered to be restrained. Unrestrained participants had a mean RS score of $7.91 \pm 2$, whereas restrained participants had a mean 
RS score of $14.33 \pm 3.1$. An independent-samples t-test demonstrated that the two groups indeed differed significantly in mean RS score, $t(44)=8.35, p<0.001$.

A 2 (Impulsivity versus Inhibition) by 2 (restrained versus unrestrained) betweensubjects ANOVA with caloric intake as the dependent variable was conducted. Because the high restrained participants had a significantly higher BMI compared to the low restrained participants, BMI was included as a covariate. BMI proved to be significant as a covariate, $F(1,41)=5.32, p<0.05$. The main effects of Condition, $F(1,41)=13.48, p \leq$ 0.001 , and Restraint, $F(1,41)=10.76, p<0.01$, were significant. Caloric intake was on average higher in the Impulsivity Condition (432 kcal $\pm 29 \mathrm{kcal})$ than in the Inhibition Condition $(287.86 \pm 32 \mathrm{kcal})$. Restrained eaters $(443.76 \pm 30 \mathrm{kcal})$ consumed significantly more calories than their unrestrained counterparts (276.61 $\pm 32 \mathrm{kcal})$. All means reported are Estimated Marginal Means \pm Standard Error of Mean. The interaction effect between impulsivity induction and Restraint was marginally significant, $F(1,41)=3.87, p<0.06$. In the Inhibition Condition there was no difference in caloric intake between the restrained and unrestrained women, $t(20)=1.1, p>0.2$. However, after the impulsivity induction the restrained women ingested significantly more calories than the unrestrained women, $t(22)=2.79, p<0.05$. See Figure 1 for an Estimated Marginal Means plot of the interaction effect between Condition and Restraint Status. 


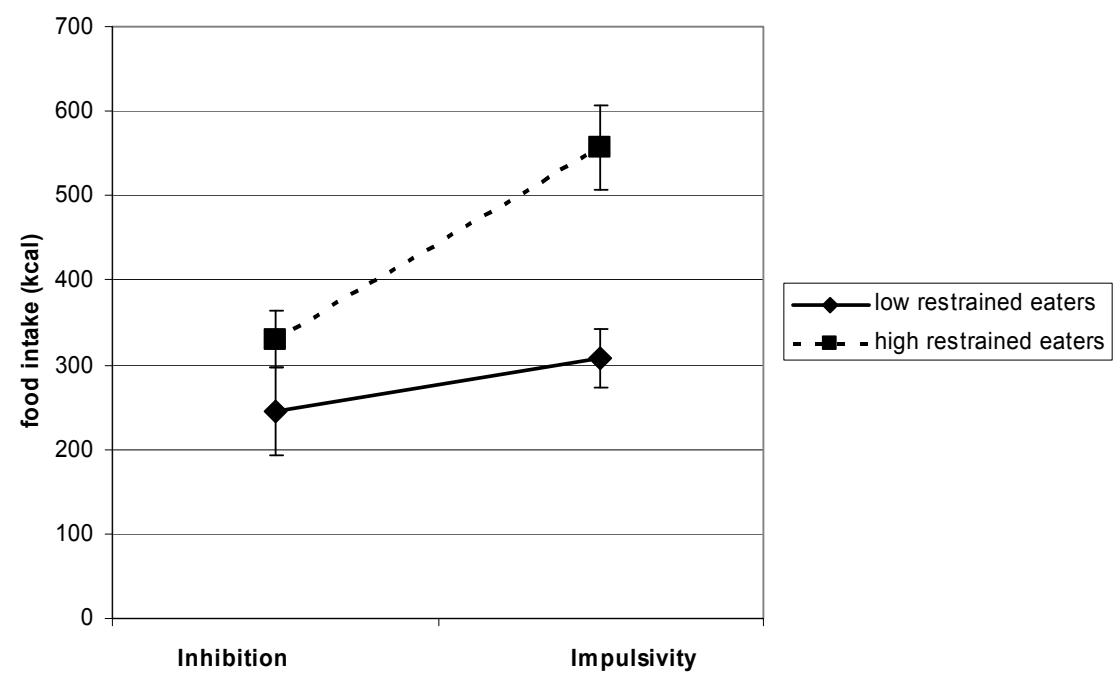

Figure 3.1

Estimated Marginal Means plot of the interaction between Condition (Inhibition versus Impulsivity) and Restraint Status (low restrained versus high restrained) on Caloric Intake, $F(1,41)=3.87$, $p<0.06$. Means are \pm SEM.

\section{Discussion}

As predicted, priming healthy participants with "impulsivity" led them to ingest significantly more calories compared to priming them with "inhibition". In other words, the hypothesis that impulsivity is causally linked to overeating was supported. To the authors' knowledge this is the first time that this hypothesis was explicitly tested and supported. This means that replication of the results, preferably with different methods and/or populations, is necessary. For this reason we decided to conduct a second study. In this study impulsivity was not manipulated by priming cognitions, but by a task that made the participants bebave in an impulsive or controlling manner.

Also as expected, the effect of the impulsivity priming on caloric intake was moderated by Restraint: the restrained participants in the Impulsivity Condition had the highest caloric intake. This outcome seems to support the notion that Restraint is indeed an important variable to consider when it comes to eating regulation in healthy participants. In this case being restrained makes the participants even more sensitive to the impulsivity priming. This makes perfect sense in Restraint Theory, since bringing partici- 
pants in an impulsive, disinhibited mood will make it more difficult to stick to their weight loss aspirations when confronted with tasty food.

A factor that was not taken into account in this study is Current Dieting. According to Restraint Theory "restrained eating" and "dieting" are essentially the same. However, Lowe (Lowe, Whitlow, \& Bellwoar, 1991; Lowe, 1995) found that restrained eating and dieting have opposite effects on eating regulation in response to a disinhibitor. In the second study both Restraint and Current Dieting were measured.

\section{Study 2}

In this study we tried to manipulate impulsivity through a behavioural task, instead of using a task to prime cognitions. We used a task that is normally used to measure impulsivity, the Stop-Signal Task (SST; Logan et al., 1997), in order to train healthy participants to react in an impulsive ("Impulsivity Condition") versus an inhibited ("Inhibition Condition") manner. The advantages of this method are twofold. First, because no cognitions are used (like "enjoy life as much as possible"), possible demand characteristics are minimized. Second, the induction is closely related to a measurement of impulsivity, which has previously been linked to overeating and obesity (Guerrieri et al., 2007b; Nederkoorn et al., 2006). Thus, the relevant aspects of the broad domain of impulsivity are manipulated.

It was hypothesized that the participants in the Impulsivity Condition would eat more during a bogus taste test compared to the participants in the Inhibition Condition. Furthermore, as a manipulation check, it was expected that the participants in the Impulsivity Condition would score higher on an impulsive state measure compared to the Inhibition Condition. Other mood states such as anger, tension, depression and fatigue were expected not to differ between conditions.

In the study that was reported earlier the effect of restraint was examined. Restraint theory implies that "restrained eating" and "dieting" can be used interchangeably (Herman \& Polivy, 1980). More recently, Lowe (Lowe et al., 1991; Lowe, 1993) has postulated a multifactorial model in which restrained eating and dieting are different factors that have different effects on eating behaviour. The first factor describes the frequency of dieting and overeating in the past, with or without actual weight changes (Lowe, 1993). The second factor reflects whether a person is currently making an effort to restrict calories in order to lose weight. It does not matter what kind of diet is undertaken, or whether the person actually loses weight (Lowe, 1993). Supporting the division of dieting 
behaviour into restrained eating and current dieting, Lowe $(1991 ; 1995)$ found that current dieters (CD's), unrestrained nondieters (UND's) and restrained nondieters (RND's) react differently to a disinhibitor in the form of a preload. Current dieters ate significantly more than restrained non-dieters and unrestrained non-dieters when not preloaded, whereas they sharply reduced their intake after a preload (Lowe et al., 1991). In the current study restraint and current dieting status were both measured. The impulsivity manipulation should bring participants in an impulsive, disinhibited state. Consequently, it should serve as a disinhibitor and have the same effect on eating regulation as the classic disinhibitor, a preload. If one sees the impulsivity manipulation in this study as a disinhibitor, then Restraint theory's predictions differ from Lowe's. According to Restraint theory both current dieters and high restrained non-dieters should overeat in response to the disinhibitor, whereas low restrained non-dieters should not. Based on the findings of Lowe (1991) we would predict that current dieters should eat more than high and low restrained non-dieters when not disinhibited. However, following the impulsivity induction current dieters should sharply reduce their intake.

In sum, this study looks at the effect of induced inhibition versus impulsivity and of dieting status on food intake. It is hypothesized that participants' dieting status will moderate their reaction to the behavioural priming of impulsivity or inhibition. Restraint Theory and Lowe's model differ in their predictions on the precise form of this interaction effect.

\section{Method}

Participants

Sixty-six female undergraduate students (mean age: $20.8 \pm 2.64$; mean BMI 22.32 \pm 2.55 ) were recruited to participate in a study on "cognitive performance and taste perception". All participants were tested individually and received course credit or a monetary reward of $€ 7.50$ for their participation. The procedure of the study was approved by the Ethical Committee of Maastricht University

\section{Materials}

The Hunger Scale contained three questions. Two questions were answered on a 100 mm VAS scale: "How hungry are you at the moment?" and "How much of your favourite food would you be able to eat at the moment?". The sum of the scores on these questions was used to calculate a hunger score. The last two items were open questions: 
"What time did you last eat?" and "When do you next expect to eat?". The first question was asked in order to check whether participants had adhered to the instruction to refrain from eating two hours before the experiment.

The Stop Signal Task (SST; Logan et al., 1997) is based on the notion that impulsive behaviour can be operationalised as a diminished ability to inhibit prepotent responses. In order to measure inhibitory control a behavioural computer task was developed (Logan et al., 1997). This computer task contains two sorts of trials: gotrials (75\%) and stoptrials (25\%). During the gotrials the participant performs a choice reaction time task: the participant learns to press a certain button as fast as possible dependent on the stimulus that is presented (an X on the right or an $\mathrm{O}$ on the left for $1500 \mathrm{~ms}$ ). This learned response has to be inhibited during the stoptrials: a tone serves as a stop signal and tells the participant not to push the button in response to the stimulus. At the start of the task the delay between the go signal ( $\mathrm{X}$ or $\mathrm{O}$ ) and the stop signal is set to $250 \mathrm{~ms}$. A tracking procedure adapts the delay dynamically depending on the participant's behaviour. If the participants inhibits successfully, the task is made more difficult by increasing the delay by $50 \mathrm{~ms}$. In the case of an unsuccessful inhibition the delay decreased by $50 \mathrm{~ms}$, making the task easier. The task consists of four blocks of 64 trials each and a practice block of ten trials. Two variables are measured: reaction time (RT) and stop delay. The stop signal reaction time (SSRT), the main independent variable, is calculated by subtracting the stop delay from the reaction time (Nederkoorn et al., 2006; Logan et al., 1997). The longer the SSRT, the more impulsive a participant is thought to be.

Instead of being used as a measurement instrument, the Stop Signal Task served as a way to induce impulsivity versus inhibition in this study. The SST consists of two concurrent, opposite tasks. First, participants are asked to push a button as quickly as possible in response to a stimulus (choice reaction time task). Second, it is their task to inhibit this response when they hear a tone that serves as a stop signal (inhibition task). It was our intention to mainly focus the participants' attention on one of these two tasks. It was explained to all participants that the stop signal task consists of two concurrent tasks. However, in the Impulsivity Condition, participants were told that the choice reaction time task was the most important task. In other words, for these participants responding as quickly as possible took precedence over inhibiting their response in reaction to a stop signal. This focus on reacting fast at the cost of inhibition was thought to bring the participants in an impulsive state. In the Inhibition Condition, participants were told that the inhibition task was the most important task. In other words, for these par- 
ticipants inhibiting their response took precedence over responding as quickly as possible. This focus on inhibiting at the cost of fast responses was thought to bring the participants in an inhibitive state. The Stop Signal Task was presented in 4 blocks of 96 trials which took about 20 minutes in total.

A Bogus Taste Test was used in order to measure food intake of the participants in an unobtrusive way. The test was conducted the same way as in Study 1, but different food was used: salted peanuts ( $\pm 1450 \mathrm{~g}$ ), M\&M's without peanuts ( $\pm 1800 \mathrm{~g}$ ), paprika flavoured crisps ( $\pm 850 \mathrm{~g}$ ) and marshmallows ( $\pm 950 \mathrm{~g})$.

The Short Form of the Profile of Mood States (POMS-SF; Schacham, 1983; Curran, Andrykowski, \& Studts, 1995) is a 37-item questionnaire that aims to measure six kinds of moods on a five-point scale: Tension-Anxiety, Anger-Hostility, Depression-Dejection, Vigor-Activity, Fatigue-Inertia and Confusion-Bewilderment. In this study the Dutch translation of the POMS-SF (Wald \& Mellenbergh, 1995) was used. This translation is shorter with 32 items that measure five moods (Confusion-Bewilderment was left out). Of all the moods that are measured by the POMS-SF Vigor-Activity seems to be linked most closely to impulsivity. Vigor-Activity has to do with excitability and positive affect (McNair, Lorr \& Droppleman, 1992). Excitability and positive affect are linked to the activation of the Behavioural Approach System (BAS) (Holzwarth \& Meyer, 2006; Beaver et al., 2006). The activation of the BAS is linked to impulsivity (Carver \& White, 1994). Four items, chosen by the authors, were added to the POMS-SF. These items were thought to measure an "impulsive mood" and formed the subscale IMP. The items were: unconstrained, impulsive, relaxed, uncontrolled.

The Barratt Impulsiveness Scale (BIS; Patton et al., 1995) is a self-report questionnaire that measures trait impulsiveness. It consists of 30 items to be rated on a 4-point scale. Scores range from 30 to 120 with higher scores indicating more impulsiveness. The BIS consists of three subscales: motor impulsiveness (acting without thinking), attentional impulsiveness (not focusing on the task at hand, cognitive instability) and nonplanning impulsiveness (lack of orientation to the future).

\section{Procedure}

At recruitment participants were asked to eat something small like a cheese sandwich two hours before the start of the experiment and to otherwise refrain from eating and drinking (except water). This was done in order to control for differing states of hunger between the conditions at the start of the experiment. At the beginning of the 
experimental session it was checked whether participants had adhered to this instruction.

At their arrival in the lab, participants started out by filling in the Hunger scale. Then participants proceeded with the Stop Signal Task. Both conditions of participants were confronted with the exact same task, but the instructions that each condition received differed. In the Impulsivity Condition the participants were told that reacting as fast as possible during the go-trials was more important than inhibiting their reaction during stoptrials. In the Inhibition Condition the participants were told the opposite: being able to inhibit their reaction during stoptrials took precedence over reacting as fast as possible during gotrials. After the Stop Signal Task, which lasted approximately 20 minutes, participants did the taste test. After the taste test the participants filled in the POMS-SF. They also reported their height and weight and they indicated whether they were on a diet or not. It was also checked whether participants had any relevant knowledge concerning the precise aims of the study. Afterwards, the participants were thanked and dismissed.

The Restraint Scale (RS) was not administered during the study. The RS scores were extracted from data that had been collected during a large university screening session about six months earlier that year.

The Barratt Impulsiveness Scale (BIS), which measures trait impulsivity, was sent to the participants by e-mail after they had been tested. Participants were asked to fill it in at home and to send it back by e-mail. The BIS was not administered at the end of the experimental session because the impulsivity / inhibition induction might otherwise have distorted the participants' trait impulsivity score.

\section{Results}

Participant Characteristics

Participants were divided into three dieting groups based on Lowe (1995). Those that claimed on the day of testing that they were on a diet, were labeled "current dieters" (CD's; $n=15$ ). The nondieting participants that scored below the median RS score 12 (a normal median for a Dutch sample) were labeled "low restrained nondieters" (LRND's; $n$ $=25$ ), whereas those that scored above the median RS score, were called "high restrained nondieters" (HRND's; $n=26$ ). Low restrained nondieters had a mean RS score of $7.44 \pm$ 3.3, whereas high restrained nondieters had a mean RS score of $16.81 \pm 4.3$. The current dieters had a mean RS score of $15.78 \pm$ 4.6. Independent-samples t-tests demonstrated 
that the mean RS score of the low restrained nondieters was significantly lower than the mean RS score of the high restrained nondieters and the current dieters, $t(49)=8.7, p<$ $0.001, t(37)=6.58, p<0.001$.

The restraint scores of high restrained non-dieters did not differ significantly of those of the current dieters, $t(38)=0.7, p>0.4$.

\section{Pre-existing Differences}

Independent-samples t-tests indicated that there were no significant differences in hunger, $t(65)=0.20, p>0.8$, age, $t(61)=0.23, p>0.8$, BMI, $t(65)=0.71, p>0.4$, and trait impulsivity (BIS scores), $t(43)=1.16, p>0.2$ between the Impulsivity Condition and the Inhibition Condition. See Table 3.3 for descriptives.

Table 3.3

Means and standard deviations for relevant variables in Inhibition vs. Impulsivity Condition in Study 2

\begin{tabular}{|c|c|c|c|c|c|c|}
\hline & & \multicolumn{2}{|c|}{ Inhibition } & \multicolumn{2}{|c|}{ Impulsivity } & \multirow[b]{2}{*}{$p$} \\
\hline & & $\mathrm{M}$ & SD & $M$ & SD & \\
\hline Hunger & & 10.88 & 3.66 & 10.68 & 4.15 & 0.85 \\
\hline BMI & & 22.15 & 2.07 & 22.59 & 2.91 & 0.48 \\
\hline Age & & 20.85 & 2.80 & 20.69 & 2.62 & 0.82 \\
\hline $\begin{array}{l}\text { Trait } \\
\text { (BIS) }\end{array}$ & Imp & 66.47 & 11.93 & 62.65 & 10.12 & 0.25 \\
\hline
\end{tabular}

One-way ANOVA's demonstrated that low restrained nondieters, high restrained nondieters and current dieters did not differ in hunger, $F(2,63)=1.40, p>0.2$, age, $F(2$, $59)=0.02, p>0.9$, and trait impulsivity scores, $F(2,41)=0.84, p>0.4$. BMI did differ $F(2$, $63)=4.25, p<0.05$. Post-hoc analyses revealed that the BMI of the low restrained nondieters differed significantly from that of the high restrained dieters, $t(49)=2.23, p<0.05$, and the current dieters, $t(38)=2.61, p<0.05$. High restrained nondieters' and current dieters' BMI did not differ significantly, $t(39)=0.96, p>0.3$. See Table 3.4 for descriptives. 
Table 3.4

Means and standard deviations for relevant variables for low restrained nondieters (LRND), high restrained nondieters (HRND), and current dieters (CD).

\begin{tabular}{|c|c|c|c|c|c|c|c|}
\hline & LRND & & HRND & & $\mathrm{CD}$ & & \\
\hline & $\mathrm{M}$ & $\mathrm{SD}$ & $\mathrm{M}$ & $\mathrm{SD}$ & $\mathrm{M}$ & $\mathrm{SD}$ & $p$ \\
\hline Hunger & 11.24 & 4.42 & 9.84 & 3.74 & 11.77 & 3.17 & 0.25 \\
\hline BMI & 21.29 & 2.29 & 22.68 & 2.19 & 23.46 & 2.93 & 0.02 \\
\hline Age & 20.77 & 2.47 & 20.84 & 2.67 & 20.67 & 3.20 & 0.98 \\
\hline $\begin{array}{l}\text { Trait } \quad \text { Imp } \\
\text { (BIS) }\end{array}$ & 65.89 & 11.10 & 61.19 & 10.81 & 64.78 & 10.74 & 0.44 \\
\hline
\end{tabular}

Adherence to the instructions that participants received

Independent-samples t-tests indicated that the participants in the Impulsivity Condition reacted significantly faster to the gotrials compared to the Inhibition Condition (Mean Reaction Time: $441.61 \mathrm{~ms} \pm 134.78 \mathrm{~ms}$ for the Impulsivity Condition versus 640.53 $\mathrm{ms} \pm 167.54 \mathrm{~ms}$ for the Inhibition Condition; $t(59)=5.14, p<0.001$ ). The participants in the Inhibition Condition had a larger percentage of successful inhibitions during stoptrials compared to the Impulsivity Condition, $62 \%$ versus $48 \%, t(64)=3.90, p<0.001$. This means that participants in both conditions did what they were asked to do: react as fast as possible for the Impulsivity Condition and inhibit as often as possible for the Inhibition Condition.

The Stop Signal Reaction Time (SSRT) was higher in the Impulsivity Condition compared to the Inhibition Condition, $173.36 \mathrm{~ms} \pm 66 \mathrm{~ms}$ versus $115.05 \mathrm{~ms} \pm 85.7 \mathrm{~ms}$, $t(59)=3, p<0.01$. When the stop-signal task is used to measure impulsivity, a higher SSRT indicates higher impulsivity. In this case a higher mean SSRT for the Impulsivity Condition indicates that the participants in the Impulsivity Condition certainly did behave in a more impulsive way during the task compared to the Inhibition Condition.

Effect of the Impulsivity versus Inhibition Condition and Dieting Status on caloric intake

We conducted a 2 (Impulsivity versus Inhibition) by 3 (LRND's versus HRND's versus CD's) between-subjects ANOVA with caloric intake as the dependent variable. Because BMI differed significantly among the dieting groups, BMI was included as a co- 
variate. BMI was not significant as a covariate, $F(1,59)=0.26, p>0.6$. The main effects of Condition, $F(1,59)=0.72, p>0.4$, and Dieting Status, $F(2,59)=0, p>0.9$, were not significant. However, the interaction between both factors was significant, $F(2,59)=5.39, p$ $<0.01$. Post-hoc tests revealed that both low and high restrained non-dieters in the Impulsivity Condition had a (marginally) significantly higher caloric intake compared to the low and high restrained non-dieters in the Inhibition Condition, $t(23)=2, p<0.06, t(24)=$ $2.12, p<0.05$. Current dieters on the other hand, did the opposite: the current dieters in the Impulsivity Condition had a significantly lower caloric intake compared to the current dieters in the Inhibition Condition, $t(13)=2.7, p<0.05$. See Figure 3.2 for an Estimated Marginal Means plot of the interaction effect between Condition and Restraint $\backslash$ Dieting Status.

A 2 (low trait impulsivity: BIS score $\leq 64$ versus high trait impulsivity: BIS score $>$ 64) by 2 (Impulsivity versus Inhibition) between-subjects ANOVA with caloric intake as the dependent variable. This was done to rule out an interaction between trait impulsivity, measured by the BIS, and the impulsivity induction. This interaction effect was not significant, $F(1,41)=0.02, p>0.8$.

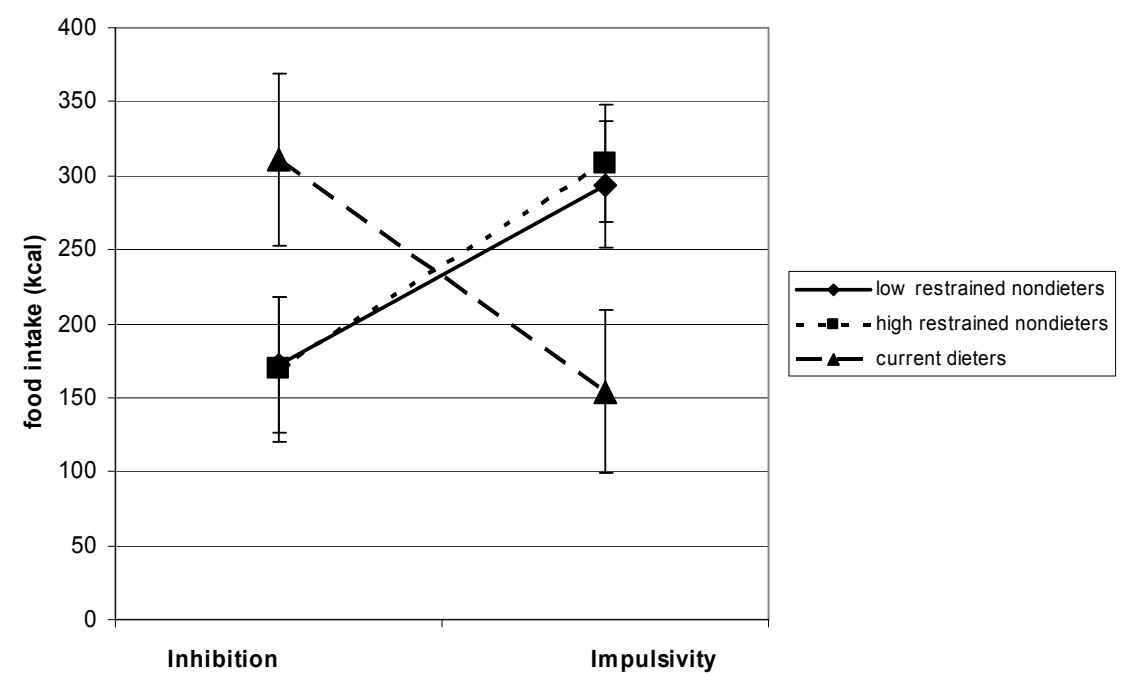

Figure 3.2

Estimated Marginal Means plot of the interaction between Condition (Inhibition versus Impulsivity) and Restraint/Dieting Status (unrestrained nondieters versus restrained nondieters versus current dieters) on Caloric Intake, $F(2,59)=5.39, p<0.01$. Means are \pm SEM. 
Manipulation Check: Effect of the Impulsivity versus Inhibition Condition on State Impulsivity and Other Mood States

As expected, the participants in the Impulsivity Condition scored higher (although marginally significantly) on the IMP subscale that was added to the POMS-SF compared to the Inhibition Condition, 6.5 versus 5.5, $t(65)=1.89, p<0.07$.

IMP was not the only subscale in which the Impulsivity Condition and the Inhibition Condition differed. The Inhibition Condition scored significantly higher on AngerHostility, 2.42 versus $0.89, t(65)=2.94, p<0.01$, and marginally significantly higher on Depression-Dejection, 1.94 versus $1.05, t(65)=1.73, p<0.10$. In order to find out what mood state differences were responsible for the interaction effect between Condition and Dieting Status, all subscale scores were centred and included as a covariate in the 2 by 2 ANOVA that yielded the significant interaction. Anger-Hostility and DepressionDejection were not significant as covariates and did not affect the significant interaction (Anger-Hostility: $F(1,58)=0.03, p>0.8$, Interaction: $F(2,58)=5.15, p<0.01$; DepressionDejection: $F(1,58)=0.09, p>0.7$, Interaction: $F(2,58)=4.99, p \leq 0.01)$. IMP was significant as a covariate $(F(1,57)=4.16, p<0.05)$. However, the interaction effect remained significant $(F(2,57)=5.03, p \leq 0.01)$. It should be noted that the linearity and noninteraction assumptions for ANCOVA analyses were checked and were not rejected.

Correlations showed that, as expected, the IMP subscale was significantly and positively related to Vigor-Activity, $r=0.46, p<0.001$. IMP was also the only subscale that was positively and significantly related to caloric intake, $r=0.34, p<0.01$. VigorActivity was marginally significantly related to caloric intake, $r=0.46, p<0.06$.

\section{Discussion}

In the current study we induced impulsivity versus inhibition by instructing participants to react in an impulsive or an inhibitory manner during the Stop-Signal Task. Analyses showed that participants indeed did adhere to our instructions. Moreover, the Impulsivity Condition scored higher on an impulsive state measure compared to the Inhibition Condition. Additionally, impulsivity was the only state measure that significantly affected caloric intake: the more impulsive the participants' state, the higher their caloric intake.

The current data matched Lowe's findings and are not in line with Restraint theory. Nondieters, high and low restrained, reacted as would be expected in the case of a causal connection between impulsivity and caloric intake: their caloric intake was the 
highest when impulsivity was induced. However, Current Dieters sharply reduced their intake when impulsivity was induced in comparison to the induction of inhibition. In other words, not Restraint, as measured by the Restraint Scale (Herman \& Polivy, 1980), but whether participants were on a diet or not had important implications for their reaction to the induction of impulsivity and inhibition. The outcome of this study is that restraint and dieting indeed seem to be different different concepts that affect eating regulation in different ways.

The fact that current dieters increased their caloric intake in response to the inhibition induction compared to the impulsivity induction might seem a counterintuitive outcome. Why would dieters eat more in reaction to an induction of inhibition? If anything, should it not help them to stick to their diet? This is not so according to the EgoStrength Model of Self-Regulation (Baumeister, Bratslavsky, Muraven, \& Tice, 1998; Muraven, Tice \& Baumeister, 1998). This model may be able to explain the dieters' behaviour. It is proposed that people have a limited capacity for self-control. Consequently, if people have exerted great amounts of self-control during one task, they are not likely to have much self-control left. Hence, their performance of another task that also demands considerable self-control will be influenced in a negative way. In a classic experiment participants that were asked to only eat radishes when chocolate cookies were also present, persisted less long at a subsequent problem-solving task (Baumeister et al., 1998). In the current study, the dieters were presumably more ego-depleted than the non-dieters. They had probably exerted more self-control by the time they arrived in the laboratory because they had a diet to stick to. The dieters in the Inhibition Condition were asked to inhibit their responses as often as possible. It is feasible that this task left them completely ego-depleted. Consequently they might not have had much self-control left to keep them from overeating when confronted with tasty food. During the impulsivity induction dieters were not asked to inhibit their reactions. This possibly left them with more self-control to resist the tasty food that was presented to them during the subsequent taste test. This would explain why the dieters ate more after the inhibition induction compared to when impulsivity was induced.

\section{General Discussion}

The two studies that were presented here attempted to manipulate impulsivity and inhibition both in a cognitive and a behavioural way in order to find support for a causal link between impulsivity and overeating. In the first study cognitive priming was 
used and besides a main effect of the impulsivity/inhibition priming an interaction between impulsivity and restraint was predicted. The results indicate that impulsivity indeed causes overeating and that this effect is even stronger in highly restrained participants. In the second study participants were categorized both according to their restraint status and their dieting status. High and low restrained non-dieters reacted the same way to the induction of impulsivity and inhibition: their caloric intake increased when impulsivity was induced. This again supports the hypothesis that impulsivity causes overeating, albeit within a certain group of participants, the non-dieters. Current dieters reacted in the opposite way: they sharply reduced their caloric intake following the impulsivity induction compared to the inhibition induction. This outcome supports Lowe's model: restraint and dieting are different concepts that affect eating regulation in different ways.

In sum, these studies lend support to the hypothesis that impulsivity is a cause of overeating. In the first study a main effect of the impulsivity induction on food intake was apparent with high restrained eaters being extra vulnerable. In the second study, when high restrained eaters were subdivided into high restrained non-dieters and current dieters, all participants except the current dieters increased their caloric intake in response to the impulsivity induction. A second important outcome is that our results support Lowe's model in the sense that the reactions of high restrained non-dieters and current dieters to the impulsivity induction mimic the reactions of Lowe's participants to a classic disinhibitor, a preload (Lowe et al., 1991).

However, there are some limitations to the studies that are presented. First, there is the lack of a (validated) manipulation check. Ideally one would use a validated state measure of impulsivity as a manipulation check when trying to induce impulsivity. If participants score higher on this measure in the experimental condition compared to the control condition, then one is sure that the manipulation led to more food intake via increased impulsivity. In the first study no manipulation check was used, whereas in the second study we used a self-devised state impulsivity measure. Using a (validated) manipulation check was very difficult in the current studies for two reasons. First, to our knowledge, a validated state measure of impulsivity does not exist. Second, the timing of the manipulation check is very difficult to determine. If one does the manipulation check immediately after the manipulation, one risks that the priming effect is already weakened once participants get to the taste test. If one does the check after the taste test, chances are that the manipulation did work during the taste test, but that the priming effect is too weak by the time participants get to the manipulation check. In the second study we 
chose to administer the manipulation check after the taste test. We did find that the Impulsivity Condition scored higher on the state impulsivity measure compared to the Inhibition Condition, although the effect was marginally significant. In future studies the timing problem could be resolved by conducting two studies: one study in which the manipulation is validated and a second study in which the effect of the manipulation on food intake is measured. However, the need for a validated state measure of impulsivity remains.

Second, when inducing a state in order to establish a causal relationship between a trait and certain behaviours it is an issue whether the state that is induced is qualitatively comparable to the trait that it is supposed to represent. In other words, is the effect of being put in an "impulsive mood" comparable to the effect of having an impulsive personality? In the area of impulsivity little attention has been paid to this issue. More work has been done in the area of anxiety. Although trait and state anxiety are measured separately (Van der Ploeg, Defares, \& Spielberger, 1980), they are considered to be closely linked. The genes that influence state and trait anxiety are identical and people with high levels of trait anxiety are more likely to become state-anxious in reaction to a situation that is seen as threatening (Lau, Eley, \& Stevenson, 2006). If this reasoning also applies to impulsivity, one could say that people with an impulsive personality are simply more prone to be in an impulsive mood. If it is indeed so that the effect of a trait on behaviour is mediated by its state, then it should not matter whether this state is induced or occurring naturally. However, further research is needed before this issue can be resolved.

In conclusion, previous research has linked impulsivity to overeating and obesity. The current study takes it a step further: impulsivity could cause overeating. With the additional support of Rotenberg and colleagues (2005) - who found that priming "lack of control" thoughts led to more food intake - it becomes plausible that we have a causal mechanism towards overweight and obesity. If further research proves this mechanism to be a valid one, then this could have consequences for the prevention and treatment of obesity. The development and validation of a training method to decrease impulsive behaviour in high risk groups might be a promising contribution to the prevention of obesity. 


\section{Part II}

The interaction between an impulsive personality and the toxic environment: its effect on overeating 



\section{Chapter 4}

How impulsiveness and variety influence food intake in a sample of healthy women

Published as: Guerrieri, R., Nederkoorn, C., \& Jansen, A. (2007 a). How impulsiveness and variety influence food intake in a sample of healthy women. Appetite, 45, 119-122. 


\begin{abstract}
This study investigates to what extent an impulsive personality, a varied food environment and their interaction influence food intake. We also investigated whether highimpulsives would exhibit more "eating disordered" thoughts and behaviours. Forty-five low-impulsives and forty-one high-impulsives did a bogus taste test with varied or monotonous food. As expected, high impulsives had a higher food intake compared to low impulsives. Moreover, high-impulsives reported significantly more "eating disordered" thoughts and behaviours than low-impulsives. Neither a main effect of variety nor an interaction between variety and impulsivity were found.
\end{abstract}

Keywords: Impulsivity; Inhibitory control; Variety; Eating disorders 


\section{Introduction}

Impulsivity is generally defined as responding with insufficient forethought, planning or control which results in an inaccurate or maladaptive action (Solanto et al., 2001). Impulsivity is associated with all kinds of pathologies ranging from addictions to ADHD (Nasser, Gluck \& Geliebter, 2004; Solanto et al., 2001). In the realm of eating behaviour and eating disorders impulsivity has also become a key concept. Research has shown that obese children (Nederkoorn, Braet, Van Eijs, Tanghe \& Jansen, 2006), obese women with Binge Eating Disorder (Nasser et al., 2004) and restrained eaters (Nederkoorn, Van Eijs \& Jansen, 2004) are more impulsive than controls. Moreover, impulsivity is a risk factor for eating disorder behaviour in adolescents (Wonderlich, Connolly \& Stice, 2004). Also, priming "lack of control" thoughts -with impulsivity being one of the priming words- led to an increased food intake in normal-weight women (Rotenberg et al., 2005). A logical interpretation of this research is that impulsive people are more vulnerable to the omnipresent temptation of tasty food in our toxic environment. Consequently, they overeat. This tendency to overeat can, in turn, lead to overweight or to the development of compensatory habits that might result in eating pathology. However, the basic assumption that impulsivity in a toxic environment leads to increased food intake is still in its infancy and previous research is incomplete for three reasons. First, actual food intake is rarely measured. Apart from Rotenberg and colleagues, we could find only one other study. Nasser and colleagues (2004) hypothesised a positive correlation between food intake and self-report impulsivity scores, but did not find it. Second, mostly clinical groups are investigated. Again, only Rotenberg and colleagues (2005) used normalweight women. If impulsivity is a cause of obesity, a relation between impulsivity and food intake should be found and replicated in normal, lean people. Third, the interaction between impulsivity and environmental factors has not been studied. The environmental factors that promote overeating are availability and palatability of food, large portion sizes and variety (Hill \& Peters, 1998; Rolls et al., 1980). Overweight children appeared more vulnerable to triggers of overeating than normal-weight children: they overate after pre-exposure to tasty food, but not without this exposure (Jansen et al., 2003). It is possible that impulsivity is the cause of this vulnerability in obese people and that impulsive people are less able to resist tempting food.

The aim of the current study was to investigate whether impulsivity of the participant and variety of the presented food influence the participants' food intake. First, we expected that participants with high impulsivity scores (behavioural as well as self- 
report) would eat more than participants with low impulsivity scores. Second, it was hypothesized that the presentation of varied food would increase food intake compared to the presentation of monotonous food. Third, the variety of the food was thought to influence the food intake of high-impulsive participants more than that of low-impulsive participants. In other words, we expected an interaction between the factors of impulsivity and variety. Fourth, it was hypothesized that impulsivity within a non-clinical population is associated with more "eating disordered" thoughts and behaviours.

\section{Method}

Participants

Eighty-six female undergraduates were recruited (mean age: 20.23 years \pm 3.35 ; mean BMI: $21.82 \pm 3$ ). All participants were randomly assigned to the variety or the monotony group and tested individually. All participants received course credit or a monetary reward.

\section{Measures}

Impulsivity was measured both by a behavioural measure, the stop-signal task (Logan et al., 1997), and a self-report measure, the Barratt Impulsiveness Scale (BIS; Patton, Stanford, \& Barratt, 1995). In the stop-signal task impulsivity is operationalised as a decreased prepotent response inhibition (Logan et al., 1997). The stop-signal reaction time (SSRT) is used as a dependent variable. The BIS measures motor impulsiveness (acting without thinking), attentional impulsiveness (not focusing on the task at hand, cognitive instability) and nonplanning impulsiveness (lack of orientation to the future) (Patton et al., 1995). Scores range from 0 to 120. "Eating disordered" thoughts and behaviours were measured by the Restraint Scale (RS; Herman \& Polivy, 1980) and the Eating Disorders Examination Questionnaire (EDE-Q; Fairburn \& Beglin, 1994). The RS assesses attitudes towards weight, degree and frequency of dieting, loss of control over eating and weight fluctuations. Scores range from 0 to 40 . The EDE-Q measures four sorts of dysfunctional thoughts and behaviours: restraint, eating concern, shape concern and weight concern. Scores range from 0 to 6 . 
Procedure

At recruitment, participants were asked to eat something small like a sandwich two hours before testing and to otherwise refrain from eating or drinking (except water). At their arrival all participants stated that they had followed instructions. Hence, we can assume that differing states of hunger prior to the taste test were controlled for.

All participants started with the stop-signal task as described by Logan and colleagues (1997). After this computer task both groups of participants received a bowl filled with approximately 1600 grams of "sugar beans". These are almond-shaped chocolate candies covered in a layer of sugar. For the variety group the bowl contained fourteen different colours of sugar beans. The monotony group was presented with an equal amount of plain white beans with identical taste and texture. Under the guise of a taste test participants were left alone for 15 minutes to taste the beans and to fill in a bogus taste perception questionnaire. After 15 minutes the experimenter returned and removed the sugar beans. The beans were weighed before and after the taste test to establish food intake in grams. While the experimenter was off weighing the beans the participants filled in the questionnaires (RS, BIS, EDE-Q). Lastly, participants were weighed and measured.

\section{Results}

A 2 (high vs. low impulsives) by 2 (variety vs. monotony) between-subjects ANOVA with the logarithmically transformed food intake as the dependent variable was conducted. When a median-split of the BIS score (=66) was used to differentiate high impulsives from low impulsives, high-impulsives ate significantly more sugar beans compared to low-impulsives, $F(1,82)=5.06, p=0.03$ (see Table 4. 1). However, variety in colour did not lead to an increase in food intake $(F(1,82)=0.357, p=0.55)$, and the interaction effect $(F(1,82)=0.685, p=0.41)$ between impulsivity and variety was not significant.

When the participants were categorized as high or low impulsive, based on the stop-signal task (median SSRT = 159.44 milliseconds), no influence of impulsivity on food intake was found $(F(1,81)=0.17, p=0.68)$, nor an interaction between impulsivity and variety $(F(1,81)=0.17, p=0.95)$.

Independent samples t-tests were conducted to find out whether high-impulsives exhibited more dysfunctional thoughts and behaviours as measured by the RS and the EDE-Q and its four subscales (see Table 1). This was the case for the total EDE score $(t(84)=2.59 ; p=0.01)$ and for three subscales of the EDE-Q: eating concern $(t(84)=2.36$; 
$p=0.02)$, shape concern $(t(84)=2.96 ; p=0.00)$ and weight concern $(t(84)=2.29 ; p=0.03)$. High and low impulsives did not differ significantly on the restraint subscale of the EDE$\mathrm{Q}(t(84)=1.84 ; p=0.07)$ and on the $\operatorname{RS}(t(84)=1.64 ; p=0.11)$.

When the participants were categorized as high or low impulsive based on the stop-signal task, as opposed to the self-report measure, no significant differences between high and low- impulsives were found, neither on the RS, nor on the total EDE-Q score or its subscales.

\section{Table 4.1}

Means and standard errors on relevant variables for high and low impulsives

\begin{tabular}{lllll}
\hline Dependent and independent & \multicolumn{2}{l}{ Low impulsives } & \multicolumn{2}{l}{ High impulsives } \\
\cline { 2 - 3 } measures & M & SEM & M & SEM \\
\hline BIS total & 59.18 & 0.80 & 74.38 & 0.83 \\
Stop Signal Reaction Time & 165.07 & 9.56 & 163.23 & 6.41 \\
Food intake (in grams) & 14.76 & 1.93 & 20.86 & 2.86 \\
RS & 10.02 & 0.82 & 12.18 & 1.05 \\
EDE-total & 1.09 & 0.13 & 1.71 & 0.19 \\
EDE-Restraint & 1.00 & 0.15 & 1.45 & 0.20 \\
EDE-Eating Concern & 0.49 & 0.08 & 0.93 & 0.17 \\
EDE-Shape Concern & 1.47 & 0.17 & 2.34 & 0.24 \\
EDE-Weight Concern & 1.40 & 0.18 & 2.11 & 0.25 \\
\hline
\end{tabular}

\section{Discussion}

The results of this study lend added credibility to the possibility that impulsivity causes people to eat more. In time, this could lead to overweight or to compensatory habits that might result in eating pathology. Previous research has shown that obese women and children are more impulsive than normal-weight controls (Nasser et al., 2004; Nederkoorn et al., 2006). The current study adds to this that impulsiveness also influences food intake in normal-weight healthy women: high-impulsive women eat more in the lab compared to low-impulsive women. High-impulsive women ingested on average an additional $27 \mathrm{kcal}$ compared to low impulsive women. According to calculations by Levitsky (2005) an average daily increase of 5 to $8 \mathrm{kcal}$ is sufficient to lead to 
considerable weight gain in the long term. Since impulsivity leads to a fourfold increase it certainly seems a relevant factor.

The hypothesis that high-impulsives would exhibit more "eating disordered" thoughts and behaviours, was partly confirmed. High-impulsive participants exhibited significantly more dysfunctional thoughts and behaviours as measured by the EDE-Q and three of its subscales than low-impulsives. For the RS and the restraint subscale of the EDE-Q a trend in the same direction was noticeable. This finding again supports the hypothesis that impulsivity is a risk factor for eating disordered behaviour such as overeating. It is in accordance with previous research by Wonderlich and colleagues (2004) who found that behaviourally measured impulsivity predicted eating disorder behaviours such as binging and purging. Of course, this does not mean that impulsivity will necessarily lead to clinical eating problems. The scores of the high-impulsive participants stayed below the cut-off scores for Bulimia Nervosa.

The effect of impulsivity on food intake and on "eating disordered" thoughts and behaviours was only found when participants were categorized based on a self-report measure of impulsivity as opposed to a behavioural measure. Lijffijt (2004) had a comparable problem: he categorized healthy participants as high or low impulsive based on a self-report measure of impulsivity. No differences in the speed of inhibiting a response were found between the groups. Lijffijt (2004) suggested that the behavioural measure was not sensitive enough to detect differences in a non-clinical population. Another possibility is that the behavioural task measures another aspect of impulsivity compared to the self-report measure. In the current study, the measures did not correlate at all $(\mathrm{r}=-$ $0.02 ; p=0.83$ ) and this lack of convergent validity between different measures of impulsivity is often reported in the literature (e.g. Wingrove \& Bond, 1997).

As far as the influence of variety on food intake is concerned, the current study could not confirm that variety in colour leads to increased food intake. Possibly, merely varying colour was not strong enough as a manipulation to affect food intake. Varying colour together with taste and/or texture might be a better option, since it has already shown to increase food intake (Rolls et al., 1980). Future research should focus on whether highly impulsive participants are more sensitive to this kind of variety in food.

In conclusion, in normal-weight, healthy women impulsivity seems to go together with a heightened food intake and with more "eating disordered" thoughts and behaviours. This is in accordance with previous research that identified impulsivity as a risk factor for eating disorder behaviour such as bingeing and purging. 



\section{Chapter 5}

The interaction between impulsivity and a varied food environment: Its influence on food intake and overweight in primary school children

Published as: Guerrieri, R., Nederkoorn, C., \& Jansen, A. (2008). The interaction between impulsivity and a varied food environment: Its influence on food intake and overweight. International Journal of Obesity, 32, 708-714. 


\section{Abstract}

Objective: The current study tests the influence of two factors, the obesogenic environment and impulsivity, on food intake in primary school children. Our current food environment offers a large variety of cheap and easily available sweet and fatty foods. This obesogenic environment is believed to be a cause of the recent obesity epidemic. Impulsive people are generally less successful at inhibiting prepotent responses and they are reward-sensitive. We investigate whether the interaction between an obesogenic environment and an impulsive person leads to overeating.

Design: a quasi-experimental 2 (reward-sensitive vs. not reward-sensitive) by 2 (successful response inhibitors vs. unsuccessful response inhibitors) by 2 (monotonous vs. varied food environment) between-subjects design with caloric intake during a taste test as the main dependent variable. The link between impulsivity and overweight was also examined.

Subjects: 78 healthy primary school children (age: 8-10y)

Measurements: We measured two aspects of impulsivity: reward-sensitivity and deficient response inhibition. Subsequently, one aspect of the obesogenic environment was manipulated: half of the participants received monotonous food during a bogus taste test whereas the other half tasted food that was varied in colour, form, taste and texture.

Results: As expected, reward-sensitivity interacted with variety: In the Monotony Group there was no difference in food intake between the less and more reward-sensitive children $(183 \mathrm{kcal} \pm 23 S D$ vs. $180 \mathrm{kcal} \pm 21 S D)$. However, in the Variety Group the more reward-sensitive children ingested significantly more calories than the less rewardsensitive children $(237 \mathrm{kcal} \pm 30 S D$ vs. $141 \mathrm{kcal} \pm 19 S D)$. Reward-sensitivity was not linked to overweight. Deficient response inhibition did not interact with variety, but it was linked to overweight.

Conclusion: It is suggested that reward-sensitivity could be a causal mechanism for overeating in an obesogenic environment whereas prepotent response inhibition may be a maintaining factor of the problem of overeating.

Keywords: response inhibition, reward-sensitivity, impulsivity, variety, food intake, children 


\section{Introduction}

In the United States and in Western Europe the prevalence of overweight and obesity is rising problematically (Flegal, 2005). Even very young children are increasingly confronted with weight problems. Although this "obesity epidemic" has many adverse health consequences little is known about its causes (Flegal, 2005; Hu, 2003). If we want to stop or even reverse this obesity epidemic, children are a potentially valuable target group. Tracking studies indicate that most children with a high BMI become adults with a high BMI (Clarke \& Lauer, 1993; Serdula, Ivery, Freedman, Williamson, \& Byers, 1993). Information about the causes of rising prevalences of childhood overweight and obesity would enable us to tackle weight problems during childhood so that we can prevent these children from becoming weighty adults.

What are potential causes of the obesity epidemic? One factor that is frequently referred to is the environment (Jeffery \& Utter, 2003). Sweet and fatty foods have never been more varied, cheaper or more available and have never been offered in larger portion sizes (Hill \& Peters, 1998). This constant confrontation with palatable food activates the hedonic system that promotes food intake not due to energetic needs, but due to environmental or emotional reasons (Lowe \& Levine, 2005). Consequently, a positive energy balance is likely, which could lead to weight gain.

However, a substantial amount of people manage to remain lean despite the temptations in our environment. This is where individual differences come into play: a person's reaction to the environment is moderated by certain character traits (Blundell et al., 2005). One such trait is impulsivity. Generally, impulsivity is defined as the tendency to think, control and plan insufficiently, resulting in an inaccurate or maladaptive response (Solanto et al., 2001). Impulsive behaviours are very diverse, but they can be narrowed down to two main aspects: reward related impulsivity and insufficient prepotent response inhibition (Solanto et al., 2001). Reward-sensitive people detect more rewarding stimuli and are more likely to approach these stimuli (Avila, 2001). Insufficient prepotent response inhibition reflects a slower response of impulsive people to inhibition signals (Barkley, 1997; Logan, Schachar, \& Tannock, 1997).

Despite these differences both aspects of impulsivity have been linked to obesity: Nederkoorn, Braet, Van Eijs, Tanghe, and Jansen (2006a) found that obese children were more reward-sensitive and worse at prepotent response inhibition than control children. Obese adult women also appeared to be worse response inhibitors than lean controls, especially toward the end of the computer task (Nederkoorn, Smulders, Havermans, 
Roefs, \& Jansen, 2006b). Moreover, insufficient response inhibition was an obstacle in the treatment of the obese children: the most impulsive children lost less weight (Nederkoorn, Jansen, Mulkens, \& Jansen, 2007). Obesity has also been linked to AD/HD, a disorder that is hallmarked by an excess of impulsive behaviour (Davis, Levitan, Smith, Tweed, \& Curtis, 2006; Agranat-Meged at al., 2005; Holtkamp et al., 2004).

However, this sort of research only allows us to conclude that impulsivity and obesity are linked. Whether impulsivity makes one obese, whether obesity makes one impulsive, or both cannot be determined. To investigate whether impulsivity could lead to overweight or obesity through overeating, Guerrieri conducted two studies with normalweight women. In the first study (Guerrieri, Nederkoorn, \& Jansen, 2007a) participants performed the Stop Signal Task which measures response inhibition (Logan et al., 1997). This task was followed by a bogus taste test. Participants were presented with a bowl of "sugar beans", almond-shaped chocolate candies covered in a layer of sugar, and were asked to taste these and to fill in a bogus taste perception questionnaire. Participants in the Variety Group received 14 different colours of sugar beans whereas the Monotony Group received an equal amount of white sugar beans. The sugar beans were thus varied in colour, but not in taste, form and texture. After the taste test participants filled out the Barratt Impulsiveness Scale (BIS; Patton, Stanford, \& Barratt, 1995), which measures three kinds of impulsive behaviour: motor impulsiveness (acting without thinking), attentional impulsiveness (not focusing on the task at hand) and nonplanning impulsiveness (lack of orientation to the future). High self-report of impulsive behaviours, but not response inhibition was associated with a significantly higher caloric intake. This effect was not moderated by variety.

The second study (Guerrieri et al, 2007b), in which variety was not manipulated, consisted of four sessions. The first day the Stop Signal Task was performed, followed by a taste test. Two days later participants returned for a second taste test, followed by a third taste test another two days later. Three to four weeks later the participants filled in self-report impulsivity measures. The results were that response inhibition as well as selfreport impulsivity significantly predicted the cumulative food intake during the three taste tests.

The aim of the current study was to find out whether impulsivity, possibly in combination with exposure to (aspects of) an obesogenic environment, leads to overeating in healthy children. We recruited a group of 78 primary school children. We measured the two aspects of impulsivity: reward-sensitivity and deficient prepotent response 
inhibition. Subsequently, one aspect of the obesogenic environment was manipulated: half of the children received monotonous food during a taste test whereas the other half tasted varied food.

We expected that response inhibition would interact with variety in the sense that poor response inhibitors would overeat especially when varied food was offered and not when bland food was offered. Davis et al. (2006) contend that poor inhibitory control could lead to unrestrained eating, especially in our obesogenic environment. In a previous study (Guerrieri et al., 2007a) we found that impulsivity measured as insufficient prepotent response inhibition did not influence food intake and did not interact with variety. However, in this study only the colour of the presented food was varied. Consequently, the manipulation may not have been strong enough. In the current study colour, taste, form and texture were all varied.

As far as reward-sensitivity is concerned, we expected that reward-sensitive children would have a difficult time resisting varied food compared to children that are relatively insensitive to reward. For monotonous food no differences were expected. Blundell et al. (2005) agree with this hypothesis: they see a high food-induced pleasure response as a behavioural risk factor for overconsumption and they also predict that this risk factor will only lead to excessive food intake in an obesogenic environment.

Based on the results of Nederkoorn et al. (2006b) we expected that the overweight children in this sample would exhibit poorer response inhibition skills than the lean children, especially toward the end of the computer task. Since the aspect of rewardsensitivity has also been linked to obesity (Nederkoorn et al., 2006a), we expected that, besides being poor response inhibitors, the overweight children would also be more reward-sensitive.

In sum, we had three hypotheses: (1) Response inhibition will interact with variety: poor response inhibitors will overeat especially when varied food is offered and not when bland food is offered (2) Reward-sensitivity will interact with variety: rewardsensitive children will overeat especially when varied food is offered and not when bland food is offered (3) The overweight children in this sample will be less effective response inhibitors and more reward-sensitive than the lean children. 
Method

Participants

Five primary schools in the area of Maastricht participated in a study on "Nutrition and Health". All children in the third and fourth grade (children between 8 and 10 years old) received a consent form. About $50 \%$ of the parents gave consent for their child to participate. The exclusion criteria were allergy or dislike of the specific candy, being on a diet or language problems. This left us with 78 eligible children between 8 and 10 years of age ( 45 boys vs. 33 girls; mean age: 9 years \pm 0.60 years; mean BMI $17.36 \pm 2.58$ ).

\section{Materials}

At the beginning of the experimental session the experimenter had a short interview with the participant asking several questions concerning hunger and last meal. Hunger was measured by asking the children how full their stomach felt at that moment. They could choose one of three options: (1) my stomach is very full; I cannot eat anything (2) my stomach is comfortably full; it is not too full, but also not empty (3) my stomach is empty; I could eat a lot of food. This led to a hunger score ranging from 0 (not hungry at all) to 2 (very hungry). The experimenter also asked the children when they had their last meal: the night before, at breakfast, during their morning break, at lunch, or during their afternoon break. The children also reported what their last meal had consisted of and age and sex were noted.

Measure of response inbibition: the Stop Signal Task (SST; Logan et al., 1997) is based on the notion that impulsive behaviour can be operationalised as a diminished ability to inhibit prepotent responses. In order to measure inhibitory control a behavioural computer task was developed (Logan et al., 1997). This computer task contains two sorts of trials: gotrials (75\%) and stoptrials (25\%). During the gotrials the participant performs a choice reaction time task: the participant learns to press a certain button as fast as possible dependent on the stimulus that is presented (an X on the right or an $\mathrm{O}$ on the left for $1500 \mathrm{~ms}$ ). This learned response has to be inhibited during the stoptrials: a tone serves as a stop signal and tells the participant not to push the button in response to the stimulus. At the start of the task the delay between the go signal ( $\mathrm{X}$ or $\mathrm{O}$ ) and the stop signal is set to $250 \mathrm{~ms}$. A tracking procedure adapts the delay dynamically depending on the participant's behaviour. If the participant inhibits successfully, the task is made more difficult by increasing the delay by $50 \mathrm{~ms}$. Following unsuccessful inhibition the delay is decreased by $50 \mathrm{~ms}$, making the task easier. The task consists of four blocks of 64 trials 
each and a practice block of ten trials. Two variables are measured: reaction time (RT) and stop delay. The stop signal reaction time (SSRT), the main independent variable, is calculated by subtracting the stop delay from the reaction time (Logan et al., 1997). The longer the SSRT, the more impulsive a participant is thought to be. In the current study we used a child-friendly version of the task (Nederkoorn et al., 2006a). Instead of an X or an $\mathrm{O}$, the stimulus was a clown that popped up at the right or at the left of the screen. Moreover, because we suspected that ten practice trials would not suffice for the children, the first block was also considered a practice block. Consequently, in the current study the Stop Signal Task consisted of three blocks instead of four. The Stop Signal Task has been administered to children starting from 6 years of age (Williams, Ponesse, Schachar, Logan, \& Tannock, 1999). Consequently, we did not expect any problems in administering this task to our sample of eight to ten-year-olds.

Measure of reward-sensitivity: the Door Opening Task aims to measure reward dominance in children aged 8 to 13 years. It is a slightly adapted version of the task used by Matthys, van Goozen, de Vries, Cohen-Kettenis, \& van Engeland (1998) that was also used by Nederkoorn et al. (2006a). The participant is told that he or she can earn points within this task and should try to collect as many points as possible. The participant earns a point when the door on the computer screen reveals a smiling face. However, when this door reveals a sad face, the participant loses a point. In total, there are 100 doors to open. The probability of getting a winning door drops from $90 \%$ to $10 \%$ as the participant opens more doors. Since the participant has to collect as many points as possible, he or she should quit opening doors once the probability of a winning door drops below 50\%. If the participant keeps opening the doors in search for reward in spite of punishment, the reward system is thought to be dominant. A participant that is easily discouraged by the encounter of a few losing doors is thought to be sensitive to punishment.

Food intake was measured via a Bogus Taste Test. In the Variety Group participants were confronted with a dish that contained five sorts of marshmallows: white-pink marshmallows ( $\pm 95 \mathrm{~g}$ ), pink marshmallows covered in coconut $( \pm 40 \mathrm{~g})$, white marshmallows covered in coconut $( \pm 40 \mathrm{~g})$, marshmallows covered in milk chocolate $( \pm 80 \mathrm{~g})$ and yellow and green marshmallows in different shapes $( \pm 90 \mathrm{~g})$. In the Monotony Group a dish with an equal amount $( \pm 350 \mathrm{~g}$ ) of the regular white-pink marshmallows was served. Participants were left alone for ten minutes and asked to taste the candy. In order to indicate how much they liked the taste of the candy, they could colour one of three faces. If 
they coloured the smiling face, this indicated that they liked the candy. If they coloured the neutral face, this indicated that they did not find it particularly palatable, but also not particularly unpalatable. If they coloured the sad face, this indicated that they did not like the taste of the candy. We did not use different types of sweets because we wanted to keep the testing situation in both groups as equal as possible. Both groups received one bowl of sweets and one taste test colouring card to report their liking of the sweets. The primary interest of the authors was not how participants rated the taste of the food, but how much they ingested of the food that was offered. Without the participants' knowledge the bowls of food were weighed before and after the taste test in order to establish food intake. The amounts eaten of each food were converted to calories. The sum of these calories was the dependent variable: total caloric intake.

Participants could drink some water if they got thirsty during the taste test. Participants were told to stay in their seat and wait for the experimenter to come back in case they finished their tasting early.

\section{Procedure}

The children were tested individually in a separate room within their school somewhere between their morning break $( \pm 10: 00$ a.m.) and their afternoon break $( \pm 2: 00$ p.m.). The experimenter went to the classroom and took the child to the test room. The experimenter started with an interview that lasted about two minutes. After the interview the experimenter explained to the child that they would play two computer games. She then gave the instructions for the Stop-Signal Task. The Stop-Signal Task took about 20 minutes to complete. After this task the child got a short break while the experimenter started up the Door Opening Task. When the child was ready to proceed, the Door Opening Task was explained. The Door Opening Task took five to ten minutes. After the Door Opening Task the laptop was put away and the marshmallows and a glass of water were put on the table. The child received instructions (see Materials) and was left alone for ten minutes. Afterwards the child was weighed and measured without shoes and heavy clothing. The child was invited to choose a small present for participating. After the session the experimenter took the child back to the classroom before picking up the next child. Each testing session lasted 40 to 45 minutes.

We certify that all applicable institutional and governmental regulations concerning the ethical use of human volunteers were followed during this research. Approval of the Maastricht University Ethical Committee was obtained. 
Statistics

Participants were randomly assigned to the Monotony or Variety Group by the tossing of a coin. Pre-existing differences between the Monotony Group and the Variety Group should be ruled out, but they were checked via independent samples t-tests and a chi-square test.

A 2 (monotony versus variety) by 2 (successful versus less successful response inhibitors) by 2 (more reward-sensitive versus less reward-sensitive) between-subjects ANCOVA with caloric intake as the dependent variable and Age as a covariate was conducted to look at the effects of variety, reward- sensitivity and response inhibition on food intake. We used a median-split (median Stop Signal Reaction Time: $174 \mathrm{~ms}$ ) to separate successful response inhibitors (SSRT $<174 \mathrm{~ms}$ ) from less successful response inhibitors (SSRT $\geq 174 \mathrm{~ms}$ ). Likewise, we used a median-split (median number of doors opened: 46) to separate more reward-sensitive (opened doors > 46) from less rewardsensitive children (opened doors $\leq 46$ ). Post-hoc tests on interactions consisted of independent-samples t-tests.

In order to look at the relationship between overweight and insufficient response inhibition, a 2 (normal weight versus overweight) by 3 (block 1 versus block 2 versus block 3 of the Stop Signal Task) mixed-model ANOVA was conducted with Stop Signal Reaction Time as the dependent variable. To determine whether the overweight children opened more doors compared to the lean children, an independent samples t-test was conducted for the factor overweight and with the number of opened doors as the dependent variable.

Unless stated otherwise, all means are expressed with their standard deviations.

\section{Results}

Pre-existing differences between the Monotony Group and the Variety Group

The amounts of boys and girls did not differ between groups, 18 girls versus 22 boys in the Monotony Group and 15 girls versus 23 boys in the Variety Group, $X^{2}(1)=$ $0.24, p>0.6$. Moreover, there were no differences in hunger (ranging from 0 to 2 ), $1 \pm$ 0.32 in the Monotony Group versus $1.05 \pm 0.32$ in the Variety Group, $t(76)=0.72, p>0.4$ and BMI, $17 \pm 2.5$ for the Monotony Group versus $17.7 \pm 2.6$ for the Variety Group, $t(76)$ $=1.3, p \geq 0.2$. There was a significant age difference between the groups: the children in the variety group ( 9.1 years \pm 0.6 ) were slightly older than the children in the monotony group (8.7 years \pm 0.5$), t(76)=3.19, p<0.01$. 
Variety, Response Inhibition, Reward-sensitivity and Food Intake

Successful response inhibitors had a SSRT of $147 \mathrm{~ms} \pm 32$ whereas unsuccessful response inhibitors had a SSRT of $233 \mathrm{~ms} \pm 64$. The two groups differed significantly in mean SSRT, $t(76)=7.56, p<0.001$. More reward-sensitive children opened 79 doors \pm 11 , whereas less reward-sensitive children opened 34 doors \pm 11 . Again, the two groups differed significantly in the number of opened doors, $t(76)=17.83, p<0.001$.

Variety did not have a main effect on ingested calories, $F(1,69)=0.1, p>0.7$. The covariate "Age" had a marginally significant effect, $F(1,69)=2.99, p<0.1(8 \mathrm{y}: 133 \mathrm{kcal}$ 65; 9 y: $193 \mathrm{kcal} 107 ; 10 \mathrm{y}: 166 \mathrm{kcal} \quad 84)$.

The main effect of Response Inbibition was not significant, $F(1,69)=0.21, p>0.6$. Likewise, the interaction between Response Inhibition and Variety was not significant, $F(1,69)=1.91, p>0.15$. Hence, hypothesis 2 was not supported. See Figure 5.1 for Estimated Marginal Means plots of the interaction effects between variety and reward responsiveness and variety and response inhibition.

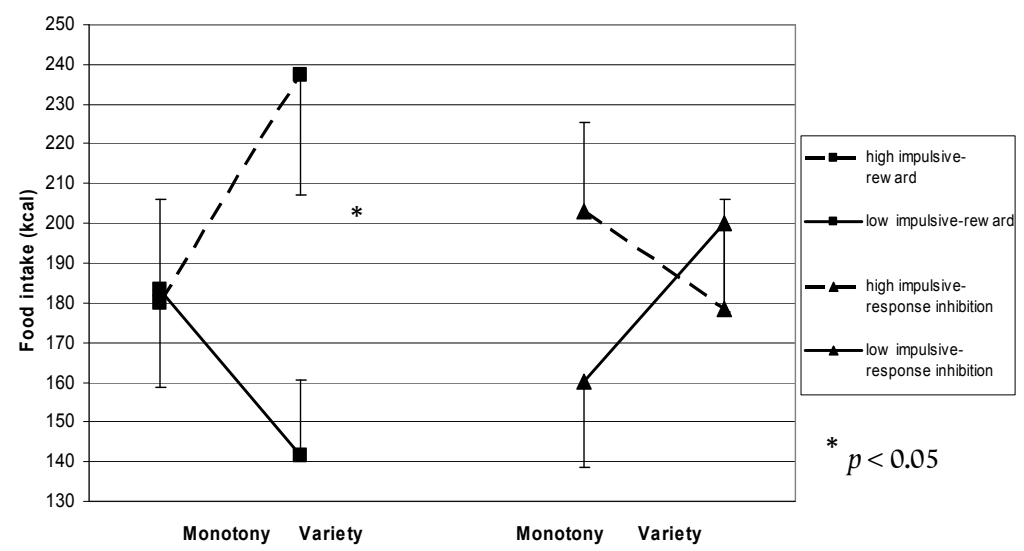

Figure 5.1

Means plot for the interaction between variety and reward-sensitivity $(F(1,69)=4.45, p<0.05)$ and variety and response inhibition $(F(1,69)=1.91, p>0.15)$. The means are \pm SEM.

The main effect of Reward-sensitivity was marginally significant, $F(1,69)=3.95, p \leq$ 0.06. Children that were reward-sensitive ingested more calories compared to children 
that were not reward-sensitive (209 kcal \pm 18 versus $162 \mathrm{kcal} \pm 14$; Estimated Marginal Means \pm SEM). The interaction between Reward-sensitivity and Variety was significant, $F(1,69)=4.45, p<0.05$. In the Monotony Group there was no difference in food intake between the less and more reward-sensitive children, $t(38)=0.27, p>0.7$. However, in the Variety Group the more reward-sensitive children ingested significantly more calories than the less reward-sensitive children, $t(36)=2.48, p<0.05$.

The three-way interaction between Variety, Response Inhibition and Rewardsensitivity did not reach significance, $F(1,69)=0.54, p>0.4$.

\section{Overweight and impulsivity}

There were 15 children in our sample of 78 who were overweight according to the national development curve (BMI overweight children $21.75 \pm 1.62$ versus BMI lean children $16.32 \pm 1.38$ ).

There was a marginally significant interaction effect between Overweight and Stop Signal Block, $F(1,76)=2.96, p<0.06$. Post-hoc tests revealed a pattern: during the first block there was no difference between normal-weight and overweight children, $t(76)=0.29, p>0.7$. However, during the second block the SSRT of the overweight children increased whereas the SSRT of the normal-weight children decreased, $t(76)=1.76$, $p<0.1$. For the third block the difference between overweight and normal-weight children is significant, $t(76)=2.14, p<0.05$. See Figure 5.2 for an Estimated Marginal Means plot.

Overweight children did not open more doors than lean children, $50 \pm 19$ doors versus $53 \pm 26$ doors, $t(76)=0.42, p>0.6$. 


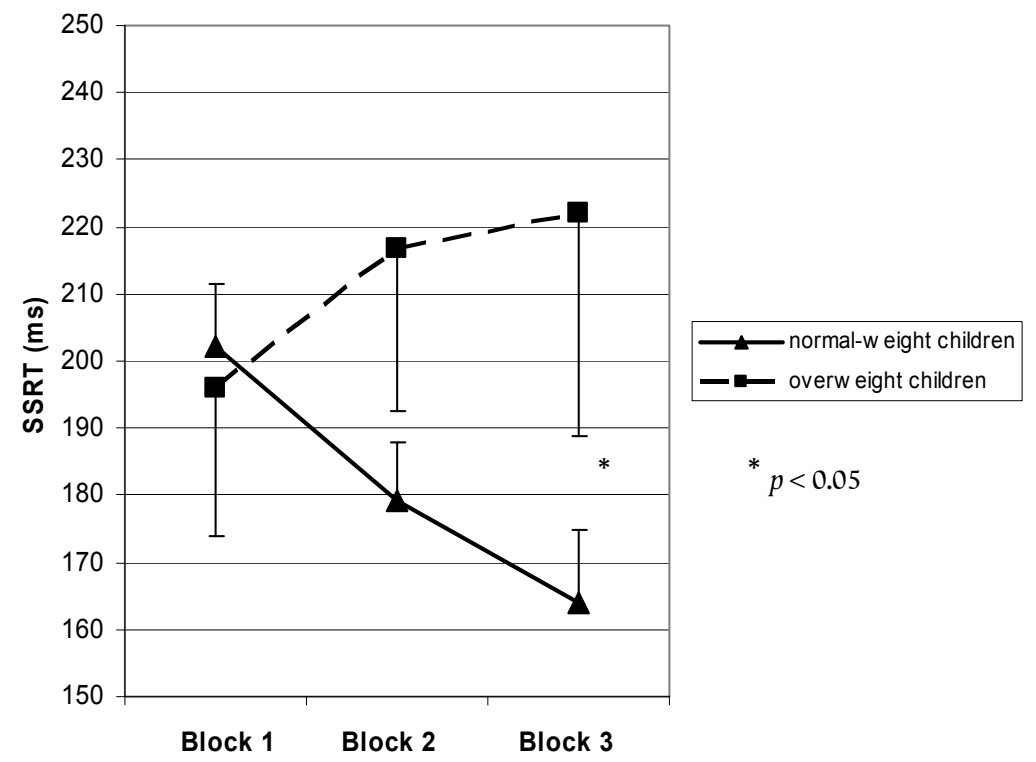

Figure 5.2

Means plot for the interaction effect between Overweight and Stop Signal Block $(F(1,76)=2.96, p$ $<0.06$ ). The means are \pm SEM.

\section{Discussion}

In the current study we tested whether two aspects of impulsivity, rewardsensitivity and response inhibition, affected food intake, especially in interaction with variety. Both aspects affected food intake quite differently.

Reward-sensitivity interacted with variety as expected. When monotonous food was offered, reward-sensitivity did not really affect caloric intake. However, when varied food was offered, reward-sensitive children ingested significantly more calories than their less reward-sensitive counterparts. In this sense the obesogenic environment, or at least the aspect of variety of this environment, could play a key role in the recent obesity epidemic. To our knowledge, this hypothesis has been formulated by multiple authors, but it was never investigated experimentally. Hence, this study is the first study in which this hypothesis is empirically supported. Due to this empirical support one can begin to take more seriously the combined effects of reward-sensitivity and variety in the food environment as a mechanism towards overweight and obesity. However, this finding 
needs to be replicated under different circumstances and in different populations in order to gain more strength as a potential obesity mechanism. Analyses of self-report rewardsensitivity and (monotonous or varied) food intake data of young women in our own databases (Guerrieri, Nederkoorn, \& Jansen, 2007, unpublished data) did lead to a replication of the results of the current study. Reward-sensitivity, measured via self-report in young women with the BIS/BAS scales (Carver \& White, 1994), interacted with variety as expected. When monotonous food (i.e. chocolates with a white sugar layer) was offered, reward-sensitivity did not really affect caloric intake. However, when varied food (i.e. the same chocolates in a variety of colours) was offered, reward-sensitive women ingested significantly more calories than their less reward-sensitive counterparts. In both studies the variety of the food was very limited. The food that is offered in our obesogenic environment is much more widely varied and as such the effect found here is probably an underestimation of the effect outside the lab.

The current study has identified a group of children who are sensitive to a varied food environment although they do not (yet?) show signs of weight problems. The next step should be a longitudinal study to investigate whether increased reward-sensitivity early in life predicts weight problems later on. If this is the case and if it is possible to teach people to deal with reward-sensitivity, this might be a useful contribution to weight loss therapies and preventive measures.

For the interaction between response inhibition and variety we expected the same pattern as for the interaction between reward-sensitivity and variety. However, it turned out to be quite different. Response inhibition did not affect food intake, and it did not matter whether monotonous or varied food was offered. This is again in accordance with one of our previous studies (Guerrieri et al., 2007a). However, in another study (Guerrieri et al., 2007b) we found that in healthy women response inhibition did predict food intake during bogus taste tests. This inconsistency is hard to explain. A main difference was that in this last study (Guerrieri et al., 2007b), the cumulated food intake over several taste tests was measured. Perhaps a single short taste test is insufficient to differentiate between people who are more or less effective at response inhibition. Perhaps the effect of less effective response inhibition is only noticeable in longer term food intake patterns.

Comparisons of the overweight children and the lean children showed that the overweight children in this study were characterized by less effective response inhibition, especially toward the end of the computer task. This is in line with the performance of the obese women in the study of Nederkoorn et al. (2006b). The performance of the 
Door Opening Task did not differ between overweight and lean children, hence in the present study there seemed to be no direct relationship between overweight and reward responsiveness. This is against our hypothesis that was based on the findings of Nederkoorn et al. (2006a). A difference between the two studies is that in the present study we tested overweight children, whereas Nederkoorn et al. (2006a) tested obese children. There is some recent evidence (Davis \& Fox, 2008) for a non-linear relationship between reward sensitivity and BMI in adult participants, but the exact form of the relationship differs from the current findings with children. Whether the association between rewardsensitivity and BMI changes with age, or whether the use of different measures of reward-sensitivity can explain these contrary findings, needs to be determined in future research.

The finding that unsuccessful response inhibition is linked to overweight, but not to food intake, could indicate that it is a mechanism that kicks in later in the process of becoming overweight or obese. It is worth investigating whether reward-sensitivity is the basic mechanism that can lead people to overeat in an obesogenic environment, whereas unsuccessful response inhibition will start to affect food intake only after a prolonged period of overeating and/or gaining weight.

Although weight problems are caused by a combination of many factors, teaching people to deal with their reward-sensitivity, and possibly their prepotent response inhibition, could bring us a step closer to solving the obesity epidemic. 
Chapter 6

General Discussion 
The general goal of the current dissertation was to contribute to research on psychological (i.e. impulsivity) and environmental (i.e. the toxic food environment) factors in the search for causal and maintaining mechanisms in the obesity epidemic. Two lines of research were set up. First, it was assessed whether impulsivity bears a causal connection to the problem of overeating. Assuming that repeated overeating, at least without an increase in energy expenditure, will in the long term lead to overweight or obesity, this could mean that impulsivity plays a role in the etiology of overweight and obesity. Second, it was investigated whether the interaction between an impulsive personality and a varied food environment is linked to excessive overeating compared to the effect on overeating of an impulsive personality per se. This could mean that healthy impulsive individuals are especially vulnerable to overeating in the current food environment that is teemed with increasingly large portions of varied, cheap and palatable sweet and fatty foods. In this section the findings of both lines of research are summarized and implications for future research and for prevention and treatment of obesity are discussed. In closing, some concluding remarks are made.

\section{Summary of the findings}

Impulsivity as a cause of overeating

Chapter 2 and 3 describe three studies in which it was attempted to induce impulsivity in healthy participants in order to see whether this induced impulsivity would influence food intake in the lab.

In chapter 2 the Scrambled Sentences Task, a priming task, was used in a withinsubjects design. The participants, 42 young healthy women, completed four sessions. Session 2 and 3 were crucial. During these sessions participants had to unscramble sentences that were either neutral in content or that hinted subtly at the construct of impulsivity. In both cases the priming task was followed by a bogus taste test to measure food intake in the lab. Although self-report craving for the served food was higher during the Impulsivity session compared to the Neutral session, actual food intake did not differ. It was contended that the impulsivity induction might have worked, but that it was not strong enough or did not last long enough to affect food intake. This problem might be solved by presenting the priming task as a memory task. More precisely, one could tell the participants that they can expect questions on the content of the 'memory' task at the end of the experimental session. The benefit of this approach is that participants have to keep the priming words in mind during the taste test. This enhances the probability of 
successful priming. In chapter 2 the sentences that were constructed during the Scrambled Sentences Task did not have to be remembered until after the taste test.

This approach, suggested in the discussion of chapter 2, was applied in the first study of chapter 3. Forty-six female undergraduate students were randomly assigned to one of two conditions. In the Inhibition condition participants read a story about Andrea's New Year's resolutions. She will start studying for her exams in time and she will save money regularly so that she will be able to go on that trip to Italy next summer. The participants in the Impulsivity Condition received a story in which Andrea says that one should not think too much about the future. Flexibility and spontaneity are more important. Andrea's only New Year's resolution is to enjoy life. In both conditions the priming task was presented as a memory task, thus participants were told to expect questions on the contents of the story they read at the end of the experimental session. In both cases the reading of the story was followed by a bogus taste test and the administration of the Restraint Scale (RS; Herman \& Polivy, 1980). The results showed a main effect of both the priming of impulsivity/inhibition and restraint status on caloric intake. Both impulsivity and a higher restraint score were associated with a higher caloric intake. Moreover, the significant interaction between the priming of impulsivity/inhibition and restraint indicated that being restrained made the participants more sensitive to the impulsivity priming.

In the second study of chapter 3 a behavioural approach was taken to induce impulsivity versus inhibition and both current dieting and restraint were measured. In order to induce impulsivity versus inhibition, 66 healthy female undergraduate students performed the stop signal task, normally used as a measure of impulsive behaviour in the form of insufficient response inhibition. All participants were informed that the stop signal task actually comprises two tasks: a go-task and a stop-task. Half of the participants were instructed to focus on the go-task (Impulsivity condition), whereas the other half of the participants were instructed to focus on the stop-task (Inhibition condition). It was demonstrated that restrained and unrestrained non-dieters acted as expected: their caloric intake was significantly higher when impulsivity was induced compared to inhibition. Current dieters sharply reduced their caloric intake following the impulsivity induction. These results are in accordance with Lowe's model (1993) that, contrary to restraint theory, states that restraint and current dieting are different constructs that affect eating regulation differently. 
The interaction between an impulsive personality and the toxic environment: its effect on overeating

Chapter 4 and 5 describe two studies in which it was investigated whether the interaction between an impulsive personality and a varied food environment is linked to excessive overeating compared to the effect of overeating on an impulsive personality per se.

In chapter 4 , impulsivity was measured in a sample of 86 female undergraduates using the BIS, a self-report measure, and the stop signal task, a behavioural task. Subsequently, the sample was subdivided in high and low impulsive individuals who were randomly assigned to a Variety or a Monotony condition. Both groups were served chocolates in a sugar layer under the guise of a taste test, but in the Variety condition the chocolates came in a variety of colours, whereas in the Monotony condition the chocolates were covered in plain white sugar. As expected, high impulsives had a higher food intake compared to low impulsives. However, these effects only occurred when the participants were characterized as high or low impulsive based on the self-report measure of impulsivity, and not on the behavioural measure. In both cases neither a main effect of variety nor an interaction between variety and impulsivity were found. The most obvious reason for the absence of a variety effect was thought to be the strength of the manipulation. In this study the only difference between the Variety and Monotony condition lay in the colour of the candy that was offered. Taste, form, and texture were kept constant.

In chapter 5 , it was made sure that the variety manipulation was more multifaceted: colour, form, taste, and texture were all varied. Furthermore, the study was conducted in a different sort of sample: primary school children between the ages of 8 and 10. We measured two aspects of impulsivity. Reward-sensitivity was measured using the door opening task and deficient response inhibition was measured using the stop signal task. Subsequently, half of the participants received monotonous food during a bogus taste test whereas the other half tasted food that was varied in colour, form, taste and texture. Deficient response inhibition did not interact with variety. Reward-sensitivity did: in the Monotony Group there was no difference in food intake between the less and more reward-sensitive children. However, in the Variety Group the more rewardsensitive children ingested significantly more calories than the less reward-sensitive children. When looking at the link between overweight and impulsivity, reward-sensitivity was not linked to overweight, whereas deficient response inhibition was. It was suggested that it is worth investigating whether reward-sensitivity is the basic mechanism 
that can lead people to overeat in an obesogenic environment, whereas unsuccessful response inhibition will start to affect food intake only after a prolonged period of overeating and/or gaining weight. In other words, reward-sensitivity could be a causal mechanism for overeating in an obesogenic environment whereas prepotent response inhibition may be a maintaining factor of the problem of overeating which will in the long term lead to overweight or even obesity.

\section{Implications for future research}

Impulsivity as a cause of overeating

From a theoretical point of view, especially the manipulation used in study 2 of chapter 3 gives rise to future research. In this study a task that is normally used to measure impulsive behaviour, the stop signal task, is used to 'train' healthy participants to react in an impulsive versus an inhibited manner. This method was inspired by the methods of some anxiety researchers. In the anxiety literature, anxiety has been experimentally induced by training normal participants to react the same way as anxious patients to the dot-probe task (Mathews \& MacLeod, 2002). In a dot-probe task two words or pictures that differ in valence are displayed simultaneously and followed by a to-be-detected target or probe. Highly anxious individuals tend to react faster to a probe in the prior location of a threatening word. This is referred to as an attentional bias toward threatening information. Mathews and MacLeod (2002) trained this attentional bias, occurring naturally in anxious patients, in normal individuals with the use of the dot-probe task. During training trials, the probe always appeared in the prior location of a threatening word for one group ("attend threat"). For the other group, the probes always appeared in the prior location of a neutral word ("avoid threat"). During test trials the "attend threat" group, similarly to anxious patients, was faster at detecting probes at the prior location of a threatening word compared to the "avoid threat" group. Moreover, after extensive training to avoid threat ("attend threat" was not possible due to ethical reasons) trait anxiety was reduced significantly in the experimental group, but not in the control group. These findings have inspired researchers to explore the clinical relevance of attentional re-training in anxiety and depression (e.g. Koster, Baert, \& De Raedt, 2006).

Attentional biases for alcohol and drug related stimuli have also been linked to the development, maintenance, and relapse of addictive behaviours and recently the first attempts have been made to re-train this attentional bias in heavy drinkers (Schoenmakers, Wiers, Jones, Bruce, \& Jansen, 2006). If one considers the method used in study 2 of 
chapter 3, one could also interpret our method as the training of an 'impulsive' bias. In this case 'bias' does not refer to an attentional bias towards certain stimuli, but to a preferred processing mode of all stimuli. More precisely, one could contend that impulsive individuals have a bias toward a fast and relatively thoughtless processing mode, whereas the processing mode of individuals high in inhibition entails being attentive toward signals of inhibition. Assuming that this is the case, the question for future research remains whether this processing bias in highly impulsive individuals could be re-trained, and whether re-training this bias would help these individuals to resist the temptation of palatable foods.

Multiple studies would be necessary for this hypothesis to gain strength. First, one would have to organise a short-term re-training of highly impulsive individuals and subsequently measure state impulsivity and food intake in the lab. This training could be very similar to the manipulation used in this dissertation: one could let participants perform the stop signal task and explicitly instruct them to focus on inhibiting successfully. Another possibility would be to re-train these individuals in a more discrete way. One could still use the stop signal task, but let the participants earn points for each successful inhibition, whereas reacting fast during go-trials does not yield any points. In this way the participants will shift their focus from reacting fast to inhibiting successfully without explicit instruction. The hypothesis would be that the participants in the experimental group would have a lower score on the state measure of impulsivity and a lower caloric intake compared to control participants. If this hypothesis would be confirmed, a second study could be conducted, testing the effect of a longer term re-training. The re-training sessions could be spread over several weeks or months. Dependent measures would include trait impulsivity and long-term food intake data, gathered through food diaries and/or more objective measures such as doubly labelled water (see Bandini, Schoeller, Cyr, \& Dietz, 1990). If this longer term training were successful in highly impulsive individuals, then it could be attempted to replicate the findings in overweight and obese groups.

From a methodological point of view, one great difficulty in conducting experimental impulsivity research is making sure that the impulsivity manipulation that one develops indeed induces impulsivity. In other words, a suitable manipulation check needs to be available. A suitable manipulation check would in this case be a validated state measure of impulsivity. If participants in the experimental group not only have a heightened caloric intake, but if they also score higher on this state measure of impulsivity, then 
it could be contended with more certainty that the manipulation led to a heightened state of impulsivity and that this heightened state of impulsivity led to a higher caloric intake in the lab. A search of the literature yielded no state self-report measures of impulsivity, except for the STIMP (STate IMPulsivity). The STIMP was designed by Wingrove and Bond (1997) and consists of 14 visual analogue scales, designed to assess impulsive mood. In an attempt to validate this measure, it was compared with scores on the $\mathrm{I}_{7}$, the BIS and the BAS subscale of the BIS/BAS scales. However, except for four significantly positive correlations between certain STIMP items and two BAS fun seeking items and two BIS items, most of the correlations were negative or very modestly positive and did not reach significance. In other words, the one self-report state impulsivity measure that was designed, could not be validated. It is thus important for future research to design and validate state measures of impulsivity that could be used as manipulation checks in research that aims to manipulate impulsivity. What form this validation should take, remains unclear. Given the multidimensional nature of impulsivity, resulting in a myriad of measures with quite weak intercorrelations (see Introduction), it seems unrealistic to expect to that a state measure of impulsivity will strongly correlate with multiple trait measures.

Another form of manipulation check is to administer a word-stem completion task or a lexical decision task. These tasks are generally used to assess schema activation (e.g. Tiggeman, Hargreaves, Polivy, \& McFarlane, 2004; Ferraro, Christopherson, \& Douglas, 2006). If we apply these tasks to experimental impulsivity research, then they could be used to assess whether impulsivity priming actually resulted in increased activation of the construct of impulsivity. If this were the case, participants primed with impulsivity should be quicker in determining whether a string of letters is an actual word if the word is related to the construct of impulsivity. Likewise, they should complete significantly more word-stems of words related to impulsivity compared to word-stems of neutral words. It is worth investigating whether these tasks can be adapted for use within impulsivity research and whether they would be useful as manipulation checks. It should be noted that a lack of effect of an impulsivity induction on these tasks does not necessarily mean that the induction was not successful. It could mean that the induction does not work via schema activation.

A third option to consider as a manipulation check is to administer a behavioural measure of impulsivity. Unlike self-report measures, behavioural measures are believed to be sensitive to short-term changes and would in this sense be suitable as a manipula- 
tion check (Moeller et al., 2001). In study 2 of chapter 3 the stop signal task was used to 'train' response inhibition or impulsivity. Following 'training', one could administer a different response inhibition task, such as the go/no-go task, without extra instructions and see whether participants trained with inhibition perform significantly better on response inhibition compared to the participants trained with impulsivity. One could even administer a reward-related task to see whether training response inhibition generalizes to sensitivity to reward. Generalization to trait impulsivity would be harder to test because it is only measured via self-report questionnaires.

In sum, theoretical implications for future research should mostly concentrate on replicating the findings with different methods and in different populations, and on the possibility of re-training highly impulsive, and possibly obese individuals. Methodologically, future research should focus on testing manipulation checks of impulsivity.

The interaction between an impulsive personality and the toxic environment: its effect on overeating

The findings in chapter 4 do not confirm that impulsive individuals are more sensitive to overeating in a varied environment. In chapter 5 not only colour, but also taste, form and texture of the offered food were varied. This manipulation did yield a significant interaction with impulsivity as measured by the door opening task. When placed in a varied environment, the high reward-sensitive participants ingested on average and additional 100 calories compared to their low reward-sensitive counterparts. At least in the case of reward-sensitivity, impulsivity seems to be the factor that leads individuals to overeat in a varied environment. However, future research is needed in three domains.

First, the findings reported in this dissertation are based on a varied environment, which is a weakened version of a toxic environment. In a toxic environment sweet and fatty foods are not only varied, but also cheap, offered in large portions and easily accessible. It is thus likely that the effect found here is an underestimation of the effects that occur in the real world. Future research should try to approximate the true toxic environment as much as possible. This might lead to even stronger interaction effects with impulsivity.

Second, it should be established which aspects of impulsivity actually lead to overeating in interaction with the toxic environment. In chapter 5 , reward-sensitivity did interact with the toxic environment, whereas deficient response inhibition did not. Theoretically not all aspects of impulsivity are expected to interact with the toxic environment 
for the same reasons or to the same extent. For example, reward-sensitivity seems a strong candidate mechanism that makes one hypersensitive to the toxic environment (Blundell et al., 2005). However, it is also plausible that a lack of planning meals leads to choosing easily accessible snack foods. Likewise, an inability to focus on the task at hand or a lack of perseverance could lead to being distracted and tempted by sweet and fatty foods when one was actually intending to buy healthy foods. In the case of adventuresomeness, for example, there would be less reason to expect that high impulsives overeat more easily in a toxic environment. In conclusion, we should find out which aspects of impulsivity lead to overeating in interaction with the toxic environment.

Third, it is possible that impulsivity causes overeating, but that repeated overeating in turn causes heightened impulsivity. In that case impulsivity could be a causal as well as a maintaining mechanism in the problem of overeating. However, these causal and maintaining mechanisms do not necessarily encompass the same aspects of impulsivity. In chapter 5, for example, the findings seem to indicate reward-sensitivity as a causal mechanism, whereas deficient response inhibition acts as a maintaining mechanism. However, in this case an alternative explanation should be ruled out first. In this study no main effect of variety was found. This leaves open the possibility that rewardsensitivity is more sensitive to variety and thus affects food intake under relatively weak conditions of variety, whereas insufficient response inhibition only affects food intake under stronger conditions of variety. Instead of varying colour, form, taste, and texture within the same food, one could thus also serve an array of entirely different foods and see whether insufficient response inhibition leads to overeating under these conditions. In conclusion, causal mechanisms should be distinguished from maintaining mechanisms. It does not suffice to investigate whether aspects of impulsivity interact with the toxic environment in healthy participants. One should also determine whether the interaction between certain aspects of impulsivity and the toxic environment only become apparent once the participants are overweight. Moreover, alternative explanations should be ruled out.

In sum, the focus of future research should be threefold. First, more aspects of the toxic environment should be involved. Second, it should be established which aspects of impulsivity actually lead to overeating in interaction with the toxic environment. Third, causal mechanisms should be distinguished from maintaining mechanisms. 
Implications for the prevention and treatment of obesity

Impulsivity as a cause of overeating

The findings in chapter 2 and 3 are quite encouraging for the hypothesis that impulsivity causes overeating. However more research is needed. Especially longitudinal studies that could demonstrate that impulsivity measured early in life predicts weight problems later in life are missing. In case of sufficient empirical support of the hypothesis that impulsivity causes overeating, possible implications for the prevention and treatment of obesity can be contemplated.

As far as prevention is concerned, the possibility of re-training the attentional bias from reacting fast toward attention for inhibition signals could be quite useful. In the United States researchers advocate obesity prevention programs in the form of schoolbased curricula that promote self-regulation and decision-making skills (Riggs, Kobayakawa Sakuma, \& Pentz, 2007). For example, children would learn to use steps that would permit them to act less impulsively with the use of a traffic light metaphor. The red light signals 'Stop-Calm down', the yellow light says 'Slow down-Think', and the green light stands for 'Go-Try my plan'. If research proves the attentional re-training described in the previous section successful, this could be integrated in this sort of program. The retraining could be adapted for children by using the children's' version of the stop signal task (see chapter 5). Furthermore, by introducing a playful element like the possibility to earn points, the re-training can be presented to the children as a computer game.

When it comes to treatment of obesity, the plot thickens. There are both arguments for and against the effectiveness of re-training in the treatment of obesity. One the one hand, one small-scale study has shown that the administration of methylphenidate to obese men led to a significant decrease in pizza intake compared to a placebo condition. Methylphenidate, or Ritalin, is normally administered to $\mathrm{AD} / \mathrm{HD}$ patients to attenuate their symptoms. One main symptom of $\mathrm{AD} / \mathrm{HD}$ patients is their increased impulsive behaviour, defined as both insufficient inhibitory control and delay discounting (e.g. Winstanley, Eagle, \& Robbins, 2006), compared to the general population. It is thus not unlikely that the effect of methylphenidate on food intake was in this case mediated by a decrease in impulsivity and that it is thus worth investigating whether decreasing impulsivity via re-training could be successful as an addition to obesity treatment. Moreover, if re-training works in healthy participants, one could contend that the margin for improvement of response inhibition is even larger in clinically obese groups. On the other hand, there are also arguments against. First, this dissertation delivers evidence for the 
hypothesis that impulsivity might cause overeating, but this does not rule out that repeated overeating might enhance impulsive behaviour. In that case, obese individuals might be very set in their ways and consequently it might be harder to re-train impulsive behaviour in this case compared to individuals who are not obese (yet). Second, in this dissertation a study was conducted in which current dieting was measured. The findings in this study could be problematic for the effectiveness of inhibition training in obese individuals on the condition that obese individuals in treatment can be labelled current dieters. Lowe (1993) defines current dieting as currently making an effort to restrict calories in order to lose weight. It does not matter what kind of diet is undertaken, or whether the person actually loses weight. In this sense, one would assume that the majority of obese adults in treatment are most likely best labelled as current dieters. However, this is still uncertain. Current dieting is established by simply asking participants whether they are on a diet to lose weight. It is thus a one-item self-report measure, which might not be very reliable or valid. One could conduct a study in which one 'trains' impulsivity versus inhibition in healthy participants who are put on a diet versus a control group who are not put on a diet. It would be interesting to see whether the participants who are put on a diet react to the training the same way as the self-defined current dieters did. That would indicate that current dieting indeed interferes with a training of impulsivity. This would have consequences for the training of inhibition in obese individuals who are currently dieting.

In this dissertation current dieters were found to drastically eat more after the inhibition induction compared to the impulsivity induction. These findings need to be clarified by conducting a study in a larger group of current dieters and with the addition of a control group that performs the stop signal task without extra instructions. This 'neutral' condition can clarify whether inhibition training leads current dieters to overeat, whether impulsivity training helps current dieters to restrict their caloric intake, or whether it is a bit of both. It has been argued before (see Discussion section chapter 5) that the EgoStrength Model of Self-Regulation (Baumeister et al., 1998; Muraven et al., 1998) may be able to explain the current dieters' behaviour. In this model it is proposed that people have a limited capacity for self-control. Consequently, if people have exerted great amounts of self-control during one task, they are not likely to have much self-control left for the performance of another self-control task. In study 2 of chapter 5, the current dieters were presumably more ego-depleted than the non-dieters. They had probably exerted more self-control by the time they arrived in the laboratory because they had a diet 
to stick to. The current dieters in the Inhibition Condition were asked to inhibit their responses as often as possible. It is feasible that this task left them completely ego-depleted. Consequently they might not have had much self-control left to keep them from overeating when confronted with tasty food. During the impulsivity induction the current dieters were not asked to inhibit their reactions. This possibly left them with more self-control to resist the tasty food that was presented to them during the subsequent taste test. This would explain why the current dieters ate more after the inhibition induction compared to when impulsivity was induced. Recently, researchers have started to investigate whether ego-depletion can be restored (Tice, Baumeister, Shmueli, \& Muraven, 2007; Gailliot et al., 2007). In this spirit one could even consider letting current dieters perform impulsivity tasks in the lab, instead of training response inhibition. If current dieters, 'trained' with impulsivity, eat significantly less compared to current dieters in a neutral condition, then it is possible that current dieters benefit from a laboratory task in which they are allowed to behave in an impulsive way and 'get it out of their system'. This might replenish their energy to stick to their diet in the real world.

In sum, there are arguments for and against re-training obese individuals as part of their treatment. Especially the findings concerning current dieters need a great amount of clarification. It is thus not (yet) recommended to re-train obese adults in treatment toward inhibition.

Obese children might be less affected by the arguments against the effectiveness of re-training as an obesity treatment component. First, although the possibility remains that repeated overeating could reinforce impulsive behaviour, compared to adults children's (impulsive) behaviour might still be more malleable (Wilson, 1994). Second, obesity treatment in children rarely involves stringent calorie restriction. Mostly, children are taught healthier eating and exercising habits with the aim being not to gain weight while growing. It is thus less certain that obese children in treatment should be labelled current dieters.

In sum, replication of the current findings and longitudinal studies should be conducted before it can be confirmed that impulsivity indeed causes overeating. If this is the case, possibilities of re-training as an addition to prevention and treatment should be explored. The potential benefits of re-training seem clearer in prevention than in treatment. 
The interaction between an impulsive personality and the toxic environment: its effect on overeating

Consensus is growing that conventional obesity treatments are not doing the job (Lowe, 2003; Hill, Thompson, \& Wyatt, 2005). Conventional therapies have improved dramatically in their ability to foster short-term weight loss. However, maintaining this weight loss seems to be an arduous task (Hill et al., 2005). Instead, researchers have started to put their faith in environmentally based approaches (Lowe, 2003; Faith, Fontaine, Baskin, \& Allison, 2007). Changing the environment in such a way that foodchoice and self-regulation are minimized, is a process that can take place on different levels. On the macro level policymakers could tax unhealthy foods and/or subsidize healthy foods in an attempt to steer the public's food choices. Another option is to make unhealthy foods less accessible and healthy foods more accessible by removing vending machines from schools and offering the children fruit instead (Faith et al., 2007). One could also modify one's food environment personally for the purpose of weight gain prevention and weight loss maintenance. Research has shown that reduced overall diet variety is an important strategy among weight loss maintainers. Another strategy to maintain weight loss is to use meal replacements (such as shakes or bars) to replace one meal or one snack a day (Lowe, 2003).

Lowe (2003) argues that the more obesogenic the environment is and the more obese or obesity-prone the individual, the greater the need to take direct control over one's personal food environment. There are two problems with this approach. First, what makes an individual obesity-prone is not explained in detail. It is merely mentioned that much of obesity-proneness is genetically based. The findings of the current dissertation suggest that impulsive individuals could be identified as obesity-prone. The study in chapter 5 has identified a group of children who are sensitive to a varied food environment although they do not (yet?) show signs of weight problems. Second, taking control over one's food environment, for example by not buying fattening foods so that they are not available in the home, still requires impulse control at the supermarket. We should thus welcome modifications of the environment in order to be forthcoming towards the needs of obesity-prone individuals, but we should not expect these modifications of the environment to get rid of all temptations and to solve the obesity epidemic on their own. It is equally, if not more important to hand the public tools to strengthen their impulse control. The 'training' of impulse control skills, as done in chapter 5 , could prove to be a useful tool. Temptation can be diminished, but it can never be banned. 
It seems that this attitude is starting to permeate the Dutch academic world. Researchers are striving for a ban on advertisements for unhealthy foods that are especially aimed at children (Havermans, Jansen, Guerrieri, \& Jansen, 2007), but at the same time it is recommended to arm children against the marketing techniques that are used in advertising (Buijzen, 2007).

\section{Concluding remarks}

In the current dissertation the findings of two lines of research were integrated. First, it was demonstrated that impulsivity in the form of response inhibition and in the form of a personality trait is one of the causes of overeating. Second, the hypothesis that impulsive - in this case reward-sensitive - individuals are more vulnerable to a varied food environment was empirically supported.

The findings throughout this dissertation demonstrate that the additional calories ingested by high impulsives compared to low impulsives, range from 27 to 145 . According to calculations by Levitsky (2005) an average daily increase of 5 to $8 \mathrm{kcal}$ is sufficient to lead to considerable weight gain in the long term. Since impulsivity leads to a fourfold increase at least and perhaps even as much as a twentyfold increase, it certainly seems a relevant factor. Consequently, tackling impulsive behaviour could indeed contribute significantly to the prevention and treatment of obesity.

The findings in this dissertation could contribute to obesity prevention and to the long-term success of its treatment in two ways. First, by delivering a means of identifying obesity-prone individuals, it becomes clearer which individuals should take direct personal control over their food environment. Second, re-training could supply both obese and obesity-prone individuals with tools to improve their impulse control. 
Summary 
Overweight and obesity rates have been rising problematically, also in the Netherlands. Today, about one in six Dutch children and more than one in two adults are overweight or obese (van den Hurk et al., 2007; CBS, 2006). The World Health Organisation (WHO, 2003) has declared obesity a global epidemic. The consequences, both for society and for overweight and obese individuals are disastrous. In the United States, for example, an estimated $9 \%$ of medical expenditures are attributable to overweight and obesity (Flegal, 2005). Moreover, at the individual level quality of life from a healthrelated, psychological and social point of view is in many cases seriously affected (Karlsson, Taft, Sjöström, Torgerson, \& Sullivan, 2003). It is clear that something needs to be done to stop or even reverse the obesity epidemic. For this reason much research has been done on causal and maintaining factors of obesity. Genetic, biological, psychological, sociocultural and ecological factors have all been suggested (Drewnowski, 1991). The research conducted for this dissertation centres around a psychological factor, impulsivity, and an environmental factor, the toxic food environment.

In chapter 1 both impulsivity and the toxic environment are defined and an overview of research that links both factors to overeating is given. Impulsivity is generally defined as the tendency to think, control and plan insufficiently, which mostly results in an inaccurate or maladaptive response (Solanto et al., 2001). However, impulsivity is considered an umbrella concept, with multiple aspects and measures of impulsivity. Impulsivity as response inhibition, impulsivity as sensitivity to reward, and impulsivity as a personality trait are used most widely. Research in obese populations, in Bulimia Nervosa patients, in $\mathrm{AD} / \mathrm{HD}$ patients, and limited research in healthy participants indicates that impulsivity and overeating are linked. Whether impulsivity actually causes people to overeat has not been investigated. In this dissertation three studies were conducted that attempted to experimentally manipulate impulsivity in healthy participants, in order to clarify the causality of the link between impulsivity and overeating. Another more established factor that leads to overeating is the toxic environment. The toxic environment is defined as a food environment that is characterized by varied, widely available, and cheap super size portions of sweet and fatty foods. A research overview indicates that both naturalistic and laboratory studies demonstrate that participants consume more food when this food is varied, when it is offered in large portion sizes and when it is easily accessible. Moreover, this effect seems to occur outside participants' awareness and without participants demonstrating a particular liking for the food that is offered. In this 
dissertation it is investigated whether placing impulsive individuals in a toxic environment leads to more overeating compared to the effect of impulsivity on overeating per se.

In sum, the aim of the current dissertation is twofold. First, an attempt is made to demonstrate the causal link between impulsivity and overeating. Second, the interaction effect of an impulsive personality and exposure to a toxic food environment on food intake is investigated. Chapter 2 and 3 centre around the causal theme, whereas chapter 4 and 5 are concerned with the interaction between an impulsive personality and a toxic environment and its effect on overeating.

In chapter 2 an attempt was made to prime the concept of impulsivity in healthy participants. In a within-subjects design one sample of female undergraduate students participated in two conditions. In both conditions participants did a priming task that either hinted subtly at the concept of impulsivity or that was neutral in content. Each time the priming task was followed by a bogus taste test. Trait impulsivity was measured by means of a behavioural measure, the stop signal task, and two self-report measures, the BIS and the $\mathrm{I}_{7}$. Firstly, we hypothesized that participants would eat more during the taste test after they had been primed with the concept 'impulsivity' compared to after the control session. Secondly, we expected that a more impulsive personality would predict a heightened food intake. Thirdly, we expected that impulsivity would predict food intake better than restraint. The results showed that both the self-report measures of impulsivity and the behavioural task predicted food intake. Moreover, restraint did not significantly predict food intake. However, primed impulsivity did not increase food intake, possibly because the priming effect did not last long enough.

Two additional methods that could potentially induce impulsivity were tested and their effect on food intake in the lab was determined in chapter 3. In the first study healthy young women were cognitively primed with the concepts 'impulsivity' or 'inhibition'. Caloric intake was significantly higher in the Impulsivity Condition compared to the Control Condition. This effect was even stronger for highly restrained participants. In the second study impulsivity was manipulated via behavioural instructions. Restrained and unrestrained non-dieters acted as expected: their caloric intake was significantly higher when impulsivity was induced compared to inhibition. Current dieters sharply reduced their caloric intake following the impulsivity induction. These results are in accordance with Lowe's model (1993) that, contrary to restraint theory, states that restraint and current dieting are different constructs that affect eating regulation differently. At least for 
non-dieters it can be concluded that heightened impulsivity versus inhibition leads to a higher food intake in the lab.

Chapter 4 consists of a study in which it is investigated to what extent an impulsive personality, a varied food environment and their interaction influence food intake. We also investigated whether high-impulsives would exhibit more 'eating disordered' thoughts and behaviours. A sample of 86 young healthy women completed the BIS, a self-report measure of impulsivity, and the stop signal task, a behavioural measure of impulsivity. Based on these measures the participants were labelled high or low impulsives. Under the guise of a taste test, both the high and low impulsive women were either served chocolates covered in a sugar layer in one of 14 different colours (variety condition) or the same chocolates covered in a white sugar layer (monotony condition). As expected, high impulsives had a higher food intake compared to low impulsives. Moreover, high impulsives reported significantly more 'eating disordered' thoughts and behaviours than low-impulsives. However, these effects only occurred when the participants were characterized as high or low impulsive based on the self-report measure of impulsivity, and not on the behavioural measure. In both cases neither a main effect of variety nor an interaction between variety and impulsivity were found. The possibility that the variety manipulation may not have been strong enough was discussed. In this study only the colour of the presented food was varied. Form, taste and texture were identical in the two groups.

In chapter 5 it was tested once more whether the interaction between a varied food environment and an impulsive person would lead to overeating. The link between impulsivity and overweight was also examined. The difference between this study and the study in chapter 3 is that the variety manipulation was made stronger (colour, form, taste, and texture were all varied) and that the sample consisted of 78 primary school children (age: 8-10y) instead of young women. We measured two aspects of impulsivity. Reward-sensitivity was measured using the door opening task and deficient response inhibition was measured using the stop signal task. Subsequently, one aspect of the obesogenic environment was manipulated: half of the participants received monotonous food during a bogus taste test whereas the other half tasted food that was varied in colour, form, taste and texture. As expected, reward-sensitivity interacted with variety: In the Monotony Group there was no difference in food intake between the less and more reward-sensitive children. However, in the Variety Group the more reward-sensitive children ingested significantly more calories than the less reward-sensitive children. 
However, reward-sensitivity was not linked to overweight. Deficient response inhibition did not interact with variety, but was linked to overweight. It is suggested that the obesogenic environment especially promotes food intake in reward-sensitive people, whereas deficient response inhibition is seen as a possible maintaining mechanism of overeating.

In chapter 6 the findings are summarized and the implications for further research and for the prevention and treatment of obesity are discussed. When it comes to research into the causality of the link between impulsivity and overeating, theoretical implications for future research should mostly concentrate on replicating the findings with different methods and in different populations, and on the possibility of re-training highly impulsive, and possibly obese individuals. Methodologically, future research should focus on testing manipulation checks of impulsivity. For the interaction between an impulsive personality and a toxic food environment, the focus of future research should be threefold. First, more aspects of the toxic environment should be involved. Second, it should be established which aspects of impulsivity actually lead to overeating in interaction with the toxic environment. Third, causal mechanisms should be distinguished from maintaining mechanisms. The findings in this dissertation could contribute to obesity prevention and to the long-term success of its treatment by delivering a means of identifying which individuals are obesity-prone and should thus take control of their food environment, and by supplying both obese and obesity-prone individuals with tools to improve their impulse control. 



\section{Samenvatting}


De prevalentie van overgewicht en obesitas rijst de pan uit, ook in Nederland. Tegenwoordig kampt ongeveer één op zes Nederlandse kinderen en meer dan één op twee volwassenen met overgewicht of obesitas (van den Hurk et al., 2007; CBS, 2006). De Wereldgezondheidsorganisatie (World Health Organisation, 2003) heeft obesitas uitgeroepen tot globale epidemie. De gevolgen, zowel voor de maatschappij als voor individuen met overgewicht of obesitas, zijn desastreus. In de Verenigde Staten bijvoorbeeld, is ongeveer 9\% van medische uitgaven door de overheid toe te schrijven aan overgewicht en obesitas (Flegal, 2005). Bovendien wordt de levenskwaliteit van individuen met overgewicht of obesitas vaak beduidend negatief beïnvloed op het vlak van gezondheid, psychologisch welzijn en sociaal functioneren (Karlsson, Taft, Sjöström, Torgerson, \& Sullivan, 2003). Het is duidelijk dat er iets moet gebeuren om de opmars van obesitas te stoppen of zelfs om te keren. Om die reden is er veel onderzoek gebeurd naar causale en in stand houdende factoren van obesitas. Een veelheid van factoren is reeds gesuggereerd: genetische, biologische, psychologische, socioculturele en ecologische (Drewnowski, 1991). Het onderzoek in dit proefschrift is gerelateerd aan een psychologische factor, impulsiviteit, en aan een ecologische factor, de toxische voedselomgeving.

In boofdstuk 1 worden zowel impulsiviteit als de toxische omgeving gedefinieerd. Bovendien krijgt de lezer in dit hoofdstuk een overzicht van het onderzoek dat beide factoren linkt aan overeten. Impulsiviteit wordt algemeen gedefinieerd als de neiging om onvoldoende na te denken, te controleren en te plannen, met als resultaat inaccurate of slecht aangepaste gedragsresponsen (Solanto et al., 2001). Werpt men echter een meer gedetailleerde blik op het concept impulsiviteit, dan ziet men dat impulsiviteit een 'parapluconcept' is. Dat wil zeggen dat er meerdere aspecten van impulsiviteit zijn, die ieder gebruik maken van hun eigen specifieke meetinstrumenten. Impulsiviteit wordt meestal geoperationaliseerd en gemeten als gebrekkige responsinhibitie, als gevoeligheid voor beloning, en als persoonlijkheidstrek. Onderzoek in obese populaties, met Bulimia Nervosa patiënten, met $\mathrm{AD} / \mathrm{HD}$ patiënten, en beperkt onderzoek met gezonde deelnemers geeft aan dat impulsiviteit gelinkt is aan overeten. Of impulsiviteit echt overeten veroor$z a a k t$, is nog niet onderzocht. Voor dit proefschrift werden drie studies uitgevoerd waarin getracht werd om impulsiviteit experimenteel te manipuleren in gezonde deelnemers, om zo meer inzicht te krijgen in het causale karakter van de link tussen impulsiviteit en overeten. Een oorzaak van overeten waar reeds meer onderzoek naar gebeurd is, is de toxische omgeving. De toxische omgeving wordt gedefinieerd als een voedselomgeving die gekarakteriseerd wordt door gevarieerde, gemakkelijk beschikbare, goedkope superpor- 
ties zoet en vet voedsel. Een onderzoeksoverzicht geeft aan dat zowel naturalistische als laboratoriumstudies laten zien dat deelnemers meer voedsel consumeren wanneer dit voedsel gevarieerd is, in grote porties wordt aangeboden, of gemakkelijk beschikbaar is. Bovendien blijken de deelnemers zich niet bewust te zijn van dit effect en is het niet eens nodig dat deelnemers het aangeboden voedsel lekker vinden. In dit proefschrift wordt onderzocht of impulsieve individuen, geplaatst in een toxische omgeving, nog gevoeliger zijn voor overeten vergeleken met impulsieve individuen in een monotone voedselomgeving.

Samengevat, is het doel van dit proefschrift tweevoudig. Ten eerste wordt er gepoogd om een causale link tussen impulsiviteit en overeten bloot te leggen. Ten tweede wordt de interactie tussen een impulsieve persoonlijkheid en de blootstelling aan een toxische voedselomgeving onderzocht. Hoofdstuk 2 en 3 gaan dieper in op de studies rond causaliteit en in hoofdstuk 4 en 5 ligt de nadruk op de interactie tussen een impulsieve persoonlijkheid en de toxische voedselomgeving en het effect hiervan op overeten.

In boofdstuk 2 werd er gepoogd om het concept impulsiviteit te primen in gezonde deelnemers. In een within-subjects design nam een steekproef van vrouwelijke eerstejaarsstudenten deel aan twee condities. In beide condities deden de deelnemers een priming taak die subtiele hints bevatte naar het concept impulsiviteit, of die neutraal was van inhoud. Na de priming taak werd iedere keer een smakentest gehouden om de voedselinname van de deelnemers te meten. Impulsiviteit werd gemeten door middel van een gedragstaak, de stoptaak, en twee zelfrapportage maten, de BIS en de $\mathrm{I}_{7}$. De eerste hypothese was dat deelnemers meer zouden eten tijdens de smakentest na de impulsiviteitspriming vergeleken met na de neutrale priming taak. Ten tweede voorspelden we dat een impulsieve persoonlijkheid een hogere voedselinname zou voorspellen. De derde hypothese was dat impulsiviteit een betere voorspeller zou zijn van voedselinname dan restraint. De resultaten gaven aan dat zowel de zelfrapportagematen van impulsiviteit als de gedragstaak een hogere voedselinname voorspelden. Bovendien was restraint geen significante voorspeller van voedselinname. Echter, geprimede impulsiviteit zorgde niet voor een toename in voedselinname, mogelijk omdat het effect niet sterk genoeg was of niet lang genoeg duurde.

Twee andere potentiële impulsiviteitsinducties werden getest en hun effect op voedselinname in het lab werd onderzocht in boofdstuk 3. In de eerste studie werden jonge, gezonde vrouwen cognitief geprimed met de concepten 'impulsiviteit' of 'inhibitie'. De voedselinname was vervolgens significant hoger in de Impulsiviteitsconditie vergeleken 
met de Inhibitieconditie. Dit effect was nog groter voor mensen die meer restrained zijn. In de tweede studie werd impulsiviteit gemanipuleerd via gedragsinstructies. Niet-lijners, restrained of niet restrained, gedroegen zich zoals verwacht: hun voedselinname lag significant hoger vergeleken wanneer impulsiviteit werd geïnduceerd vergeleken met inhibitie. Mensen die op het moment van testen aan de lijn deden, aten dan weer significant minder wanneer impulsiviteit geïnduceerd werd vergeleken met inhibitie. Deze resultaten ondersteunen het model van Lowe (1993) dat, in tegenstelling tot restraint theorie, beweert dat restraint en op het moment van testen aan de lijn doen verschillende constructen zijn die de regulatie van onze voedselinname op een verschillende manier beïnvloeden. Op zijn minst kan voor niet-lijners geconcludeerd worden dat een verhoogde impulsiviteit versus inhibitie tot een hogere voedselinname in het lab leidt.

Hoofdstuk 4 omvat een studie waarin onderzocht wordt in welke mate een impulsieve persoonlijkheid, een gevarieerde voedselomgeving en hun interactie voedselinname beïnvloeden. We onderzochten ook of hoog impulsieve deelnemers meer disfunctionele eetgedragingen en -gedachten vertoonden. Een steekproef van 86 jonge, gezonde vrouwen vulde de BIS, een zelfrapportagemaat voor impulsiviteit, in en deed de stoptaak, een gedragstaak voor impulsiviteit. Gebaseerd op hun scores op deze maten werden de deelnemers als hoog of laag impulsief bestempeld. Onder het mom van een smakentest kregen zowel de hoog als de laag impulsieve vrouwen suikerbonen in 14 verschillende kleuren te proeven (variatieconditie) of dezelfde hoeveelheid witte suikerbonen (monotone conditie). Zoals verwacht hadden de hoog impulsieve deelnemers een hogere voedselinname vergeleken met de laag impulsieve deelnemers. Bovendien rapporteerden de hoog impulsieve deelnemers significant meer disfunctionele eetgedragingen en -gedachten vergeleken met laag impulsieve deelnemers. Echter, deze effecten deden zich alleen voor wanneer de deelnemers als hoog of laag impulsief bestempeld werden, gebaseerd op hun score op de zelfrapportagemaat, en niet op hun score op de gedragsmaat van impulsiviteit. In beide gevallen werd er bovendien noch een hoofdeffect van variatie, noch een interactie effect tussen variatie en impulsiviteit gevonden. De mogelijkheid dat de variatiemanipulatie misschien niet sterk genoeg was om te leiden tot een effect op voedselinname is niet ondenkbaar. In deze studie werd immers alleen de kleur van het gepresenteerde voedsel gevarieerd. De vorm, smaak en textuur van het voedsel was identiek in beide condities.

In boofdstuk 5 werd nogmaals getest of de interactie tussen een gevarieerde voedselomgeving en een impulsieve persoonlijkheid leidt tot overeten. De link tussen impulsi- 
viteit en overgewicht werd ook onderzocht. Deze studie verschilt van de studie in hoofdstuk 3 in de zin dat de gevarieerde voedselomgeving sterker gemanipuleerd werd (kleur, vorm, smaak en textuur werden allemaal gevarieerd). Bovendien bestond de steekproef in dit geval uit 78 kinderen van 8 tot 10 jaar en niet uit vrouwelijke studenten. Twee aspecten van impulsiviteit werden gemeten. Gevoeligheid voor beloning werd gemeten aan de hand van de deuropeningstaak en gebrekkige responsinhibitie werd gemeten aan de hand van de stoptaak. Daarna werd één aspect van de toxische omgeving gemanipuleerd: de helft van de deelnemers kregen monotoon voedsel voorgeschoteld tijdens een smakentest terwijl de andere helft voedsel proefde dat gevarieerd was in kleur, vorm, smaak en textuur. Zoals verwacht interacteerde gevoeligheid voor beloning met variatie. In de groep die monotoon voedsel kreeg, was er geen verschil tussen kinderen die meer en minder gevoelig waren voor beloning, maar in de groep die gevarieerd voedsel kreeg, aten de beloningsgevoelige kinderen significant meer dan de kinderen die minder beloningsgevoelig waren. Een relatie tussen overgewicht en beloningsgevoeligheid was er echter niet. Gebrekkige responsinhibitie op zijn beurt interacteerde dan weer niet met variatie om zo voedselinname te beïnvloeden, maar was wel gerelateerd aan overgewicht. Een suggestie is dat de obesogene omgeving vooral meer voedselinname stimuleert in beloningsgevoelige mensen, terwijl gebrekkige responsinhibitie gezien wordt als een mogelijk in stand houdend mechanisme van overeten.

In Hoofdstuk 6 worden de bevindingen samengevat en de implicaties voor verder onderzoek en voor de preventie en behandeling van obesitas bediscussieerd. Wat causaliteitsonderzoek betreft, zijn de theoretische implicaties voor verder onderzoek vooral gericht op het repliceren van de bevindingen van dit proefschrift, gebruik makend van verschillende methoden en in verschillende populaties. Bovendien dient de mogelijkheid van een her-training van hoog impulsieve en mogelijk obese individuen verder onderzocht te worden. Methodologisch gezien dient toekomstig onderzoek zich te focussen op het testen van manipulatiechecks van impulsiviteit. Voor de interactie tussen een impulsieve persoonlijkheid en de toxische voedselomgeving zijn er drie aandachtspunten. Ten eerste moeten meer aspecten van de toxische omgeving betrokken worden in onderzoek. Ten tweede moet er vastgesteld worden welke aspecten van impulsiviteit nu precies tot overeten leiden in interactie met de toxische omgeving. Ten derde moeten causale mechanismen onderscheiden worden van in stand houdende mechanismen. De bevindingen in dit proefschrift kunnen op twee manieren een bijdrage leveren aan de preventie van obesitas en aan het lange-termijn succes van obesitasbehandelingen. Ten eerste door een 
middel te verschaffen aan de hand waarvan obesitasgevoelige personen geïdentificeerd kunnen worden. Deze individuen kan men dan aansporen om controle te nemen over hun voedselomgeving. Ten tweede door zowel obese als obesitasgevoelige individuen manieren aan te reiken om hun impulscontrole te verbeteren. 
References 
Agranad-Meged, A. N., Deitcher, C., Goldzweig, G., Leibenson, L., Stein, M., \& GaliliWeisstub, E. (2005). Childhood obesity and Attention Deficit/Hyperactivity Disorder: A newly described comorbidity in obese hospitalized children. International Journal of Eating Disorders, 37, 357-359.

Altfas, J. R. (2002). Prevalence of attention deficit/hyperactivity disorder among adults in obesity treatment. BMC Psychiatry, 2, 1-8.

Anderson, P. M., \& Butcher, K. F. (2006). Childhood obesity: Trends and potential causes. The Future of Children, 16, 19-45.

Araya, T., Akrami, N., Ekehammar, B., \& Hedlund, L. (2002). Reducing prejudice through priming of control-related words. Experimental Psychology, 49, 222-227.

Avila, C. (2001). Distinguishing BIS-mediated and BAS-mediated disinhibition mechanisms: A comparison of disinhibition models of Gray $(1981,1987)$ and of Patterson and Newman (1993). Journal of Personality and Social Psychology, 80, 31 1-324.

Band, G. P. H., \& van Boxtel, G. J. M. (1999). Inhibitory motor control in stop paradigms: review and reinterpretation of neural mechanisms. Acta Psychologica, 101, 179-2 11.

Band, G. P. H., van der Molen, M. W., Overtoom, C. C. E., \& Verbaten, M. N. (2000). The ability to activate and inhibit speeded responses: Separate developmental trends. Journal of Experimental Child Psychology, 75, 263-290.

Bandini, L. G., Schoeller, D. A., Cyr, H. N., \& Dietz, W. H. (1990). Validity of reported energy intake in obese and nonobese adolescents. American Journal of Clinical Nutrition, 52, 421-425.

Bargh, J. A., Gollwitzer, P. M., Lee-Chai, A., Barndollar, \& K., Trötschel, R. (2001). The automated will: Nonconscious activation and pursuit of behavioral goals. Journal of Personality and Social Psychology, 81, 1014-1027.

Barkley, R. A. (1997). Behavioral inhibition, sustained attention, and executive functions: Constructing a unifying theory of ADHD. Psychological Bulletin, 121, 65-94.

Baumeister, R. F., Bratslavsky, E., Muraven, M. \& Tice, D. M. (1998). Ego-depletion: Is the active self a limited resource? Journal of Personality and Social Psychology, 74, 12521265.

Beaver, J. D., Lawrence, A. D., van Ditzhuijzen, J., Davis, M. H., Woods, A., \& Calder, A. J. (2006). Individual differences in reward drive predict neural responses to images of food. Journal of Neuroscience, 26, 5160-5166. 
Bechara, A., \& Damasio, H. (2002). Decision-making and addiction (part I): impaired activation of somatic states in substance dependent individuals when pondering decisions with negative future consequences. Neuropsychologia, 40, 1675-1689.

Bechara, A., Damasio, A. R., Damasio, H., \& Anderson, S. W. (1994). Insensitivity to future consequences following damage to human prefrontal cortex. Cognition, 50, 7-15.

Berry, S. L., Beatty, W. W., \& Klesges, R. C. (1985). Sensory and social influences on ice cream consumption by males and females in a laboratory setting. Appetite, 6, 4145.

Blundell, J. E., \& Gillett, A. (2001). Control of food intake in the obese. Obesity Research, 9, 263S-270S.

Blundell, J. E., Stubbs, R. J., Golding, C., Croden, F., Alam, R., Whybrow, S., et al. (2005). Resistance and susceptibility to weight gain: Individual variability in response to a high-fat diet.

Bonato, D. P., \& Boland, F. J. (1983). Delay of gratification in obese children. Addictive Bebaviors, 8, 7 1-74.

Buijzen, M. (2007). Commercie in de jeugdcultuur: vette verleiders en weerbare kinderen [Commerce and youth: tempting fat foods and arming children]. In H. Dagevos \& G. Munnichs (Eds.), De obesogene samenleving. Maatschappelijke perspectieven op overgewicht (pp. 95-102). Amsterdam, the Netherlands: Amsterdam University Press

Carrillo-de-la-Peña, M. T., Otero, J. M., \& Romero, E. (1993). Comparison among various methods of assessment of impulsiveness. Perceptual and Motor Skills, 77, 567-575.

Carver, C. S., \& White, T. L. (1994). Behavioral inhibition, behavioral activation, and affective responses to impending reward and punishment: The BIS/BAS scales. Journal of Personality and Social Psychology, 67, 319-333.

Central Bureau Statistics (2006). Gerapporteerde gezondheid en levensstijl [self-reported bealth and lifestyle]. Retrieved November 2, 2007, from http://statline.cbs.nl/StatWeb/table. asp?PA=03799\&D1=267-270\&D2=0-1 7\&D3=0\&D4=a\&DM=SLNL\&LA=nl\&TT=2

Chalmers, D. K., Bowyer, C. A., \& Olenick, N.L. (1990). Problem drinking and obesity: A comparison in personality patterns and life-style. The International Journal of the Addictions, 25, 803-817. 
Claes, L., Nederkoorn, C., Vandereycken, W., Guerrieri, R., \& Vertommen, H. (2006). Impulsiveness and lack of inhibitory control in eating disorders. Eating Bebaviors, 7, 196-203.

Claes, L., Vandereycken, W., \& Vertommen, H. (2002). Impulsive and compulsive traits in eating disordered patients compared with controls. Personality and Individual Differences, 32, 707-714.

Clarke, W. R. \& Lauer, R. M. (1993). Does childhood obesity track into adulthood? Clinical Reviews in Food Science and Nutrition, 33, 423-430.

Curran, S. L., Andrykowski, M. A. \& Studts, J. L. (1995). Short Form of the Profile of Mood States (POMS-SF): Psychometric information. Psychological Assessment, 7, 80-83.

Davis, C., \& Claridge, G. (1998). The eating disorders as addiction: A psychobiological perspective. Addictive Behaviors, 23, 463-475.

Davis, C., \& Fox, J. (2008). Sensitivity to reward and body mass index (BMI): Evidence for a non-linear relationship. Appetite, 50, 43-49.

Davis, C., Levitan, R. D., Muglia, P., Bewell, C., \& Kennedy, J. L. (2004a). Decision-making deficits and overeating: A risk model for obesity. Obesity Research, 12, 929-935.

Davis, C., Levitan, R. D., Smith, M., Tweed, S., \& Curtis, C. (2006). Associations among overeating, overweight, and attention deficit/hyperactivity disorder: A structural equation modelling approach. Eating Bebaviors, 7, 266-274.

Davis, C., Patte, K., Levitan, R., Reid, C., Tweed, S., \& Curtis, C. (2007). From motivation to behaviour: A model of reward sensitivity, overeating, and food preferences in the risk profile for obesity. Appetite, 48, 12-19.

Davis, C., Strachan, S., \& Berkson, M. (2004b). Sensitivity to reward: implications for overeating and overweight. Appetite, 42, 131-138.

Davis, C., \& Woodside, D. B. (2002). Sensitivity to the rewarding effects of food and exercise in the eating disorders. Comprebensive Psychiatry, 43, 189-194.

Dickman, S. J. (1990). Functional and dysfunctional impulsivity: Personality and cognitive correlates. Journal of Personality and Social Psychology, 58, 95-102.

Diliberti, N., Bordi, P. L., Conklin, M. T., Roe, L. S., \& Rolls, B. J. (2004). Increased portion size leads to increased energy intake in a restaurant meal. Obesity Research, 12, 562567. 
Dougherty, D. M., Bjork, J. M., Harper, R. A., Marsh, D. M., Moeller, F. G., Mathias, C. W., et al. (2003). Behavioral impulsivity paradigms: a comparison in hospitalized adolescents with disruptive behavior disorders. Journal of Child Psychology and Psychiatry, 44, $1145-1157$.

Dougherty, D. M., Moeller, F. G., Steinberg, J. L., Marsh, D. M., Hines, S. E., \& Bjork, J. M. (1999). Alcohol increases commission error rates for a continuous performance test. Alcobolism: Clinical and Experimental Research, 23, 1342-1351.

Drewnowski, A. (1991). Obesity and eating disorders: Cognitive aspects of food preference and food aversion. Bulletin of the Psychonomic Society, 29, 261-264.

Drewnowski, A. (2004). Obesity and the food environment. American Journal of Preventive Medicine, 27, 154-162.

Enticott, P. G., Ogloff, J. R. P., \& Bradshaw, J. L. (2006). Associations between laboratory measures of executive inhibitory control and self-reported impulsivity. Personality and Individual Differences, 41, 285-294.

Eysenck, S. G. G., Eating, G., \& Pearson, P. R. (1984). Age norms for impulsiveness, venturesomeness and empathy in children. Personality and Individual Differences, 5, 315-321.

Eysenck, H. J. (1990). Biological dimensions of personality. In L.A. Pervin (Ed.), Handbook of personality: Theory and research (pp.244-276). New York: Guilford Press.

Eysenck, S. B. G., Pearson, P. R., Easting, G., \& Allsopp, J. F. (1985). Age norms for impulsiveness, venturesomeness and empathy in adults. Personality and Individual Differences, 6, 613-619.

Fahy, T., \& Eisler, I. (1993). Impulsivity and eating disorders. British Journal of Psycbiatry, 162, 193-197.

Fairburn, C. G., \& Beglin, S. J. (1994). Assessment of eating disorders: Interview or selfreport questionnaire? International Journal of EatingDisorders, 16, 363-370.

Faith, M. S., Fontaine, K. R., Baskin, M. L., \& Allison, D. B. (2007). Toward the reduction of population obesity: Macrolevel environmental approaches of the problems to food, eating and obesity. Psychological Bulletin, 133, 205-226.

Fassino, S., Leombruni, P., Pierò, A., Abbate-Daga, G., \& Rovera, G. G. (2003). Mood, eating attitudes, and anger in obese women with and without Binge Eating Disorder. Journal of Psychosomatic Research, 54, 559-566. 
Ferraro, F. R., Christopherson, K., \& Douglas, J. (2006). Lexical Decision Task performance in blood-fearful and spider-fearful individuals. Current Psychology: Developmental-Learning-Personality-Social, 25, 132-143.

Fischer, S., Smith, G. T., \& Anderson, K. G. (2003). Clarifying the role of impulsivity in Bulimia Nervosa. International Journal of Eating Disorders, 33, 406-411.

Flegal, K. M. (2005). Epidemiologic aspects of overweight and obesity in the United States. Physiology and Bebavior, 86, 599-602.

Fleming, J., \& Levy, L. (2002). Eating disorders in women with AD/HD. In P.O. Quinn, \& K. G. Nadeau (Eds.), Gender issues and AD/HD: Research, diagnosis, and treatment (pp. 411-426). Silver Spring, MD: Advantage Books.

Gailliot, M., Baumeister, R. F., De Wall, C. N., Maner, J. K., Plant, E. A., Tice, D. M., et al. (2007). Self-control relies on glucose as a limited energy source: Willpower is more than a metaphor. Journal of Personality and Social Psychology, 92, 325-336.

Gray, J. A. (1987). Perspectives on anxiety and impulsivity: A commentary. Journal of Research in Personality, 21, 493-509.

Guerrieri, R., Nederkoorn, C., \& Jansen, A. (2007a). How impulsiveness and variety influence food intake in a sample of healthy women. Appetite, 48, 119-122.

Guerrieri, R., Nederkoorn, C., Stankiewicz, K., Alberts, H., Geschwind, N., Martijn, C., et al. (2007b). The influence of trait and induced state impulsivity on food intake in normal-weight healthy women. Appetite, 49, 6-73.

Havermans, R. C., Jansen, E., Guerrieri, R., \& Jansen, A. (2007). Dikke kijkbuiskinderen, of waarom kinderen dik worden van televisie kijken. [Childhood obesity and television watching]. De Psycboloog, 42, 582-88.

Hedley, A. A., Ogden, C. L., Johnson, C. L., Carroll, M. D., Curtin, L. R., \& Flegal, K. M. (2004). Prevalence of overweight and obesity among US children, adolescents, and adults, 1999-2002. Journal of the American Medical Association, 291, 2847-2850.

Herman, C. P., \& Polivy, J. (1980). Restrained Eating. In A. J. Stunkard (ed.), Obesity (pp. 208-225). Philadelphia: Saunders.

Higgins, E. T., Rholes, W. S., \& Jones, C. R. (1977). Category accessibility and impression formation. Journal of Experimental Social Psychology, 13, 141-157.

Hill, J. O., \& Peters, J. C. (1998). Environmental contributions to the obesity epidemic. Science, 280, $1371-1374$.

Hill, J. O., Thompson, H., \& Wyatt, H. (2005). Weight maintenance: What's missing? Journal of the American Dietetic Association, 105, S63-S66. 
Holtkamp, K., Konrad, K., Müller, B., Heussen, N., Herpertz, S., Herpertz-Dahlmann, B., \& Hebebrand, J. (2004). Overweight and obesity in children with Attention Deficit/Hyperactivity Disorder. International Journal of Obesity, 28, 685-689.

Holzwarth, K. \& Meyer, T. D. (2006). The dysregulation of the "Behavioural Activation System": An independent dimension. Personality and Individual Differences, 41, 319328.

Hu, F. B. (2003). Overweight and obesity in women: Health risks and consequences. Journal of Women's Health, 12, 163-172.

Jansen, A. \& van den Hout, M. (1991). On being led into temptation: "Counterregulation" of dieters after smelling a "preload". Addictive Bebaviors, 16, 247-253.

Jansen, A. (1996). How restrained eaters perceive the amount they eat. British Journal of Clinical Psychology, 35, 381-392.

Jansen, A., Theunissen, N., Slechten, K., Nederkoorn, C., Boon, B., Mulkens, S. \& Roefs, A. (2003). Overweight children overeat after exposure to food cues. Eating Bebaviors, 4, 197-209.

Jeffery, R. W., \& Utter, J. (2003). The changing environment and population obesity in the United States. Obesity Research, 11, 12S-22S.

Kane, T. A., Loxton, N. J., Staiger, P. K., \& Dawe, S. (2004). Does the tendency to act impulsively underlie binge eating and alcohol use problems? An empirical investigation. Personality and Individual Differences, 36, 83-94.

Karlsson, J., Taft, C., Sjöström, L., Torgerson, J. S., \& Sullivan, M. (2003). Psychosocial functioning in the obese before and after weight reduction: construct validity and responsiveness of the Obesity-related Problems scale. International Journal of Obesity, 27, 617-630.

Koster, E. H. W., Baert, S., \& De Raedt, R. (2006). Aandachtstraining bij angst en depressie: Een wetenschappelijke innovatie met klinische relevantie [Attentions training by fear and depression: A scientific innovation with clinical relevance]. Gedragstherapie, 39, 243-255.

Kral, T. V. E., \& Rolls, B. J. (2004). Energy density and portion size: their independent and combined effects on energy intake. Physiology and Bebavior, 82, 131-138.

Kühnen, U., \& Hannover, B. (2000). Assimilation and contrast in social comparisons as a consequence of self-construal activation. European Journal of Social Psychology, 30, 799-811. 
Lau, J. Y. F., Eley, T. C., Stevenson, J. (2006). Examining the state-trait anxiety relationship: A behavioural genetic approach. Journal of Abnormal Cbild Psychology, 34, 19-27.

Lean, M. (1998). Clinical bandbook of weight management. London: Martin Dunitz.

Levitsky, D. A. (2005). The non-regulation of food intake in humans: Hope for reversing the epidemic of obesity. Physiology and Behavior, 86, 623-632.

Logan, G. D., \& Cowan, W. B. (1984). On the ability to inhibit thought and action: A theory of an act of control. Psychological Review, 91, 295-327.

Logan, G. D., Schachar, R. J., \& Tannock, R. (1997). Impulsivity and inhibitory control. Psychological Science, 8, 60-64.

Lowe, M. R. (1993). The effects of dieting on eating behavior: A three-factor model. Psychological Bulletin, 114, 100-121.

Lowe, M. R. (1995). Restrained eating and dieting: Replication of their divergent effects of eating regulation. Appetite, 25, 115-118.

Lowe, M. R. (2003). Dieting: False hope or falsely accused? American Psychologist, 58, 819820.

Lowe, M. R., \& Levine, A. S. (2005). Eating motives and the controversy over dieting: Eating less than needed versus less than wanted. Obesity Research, 13, 797-806.

Lowe, M. R., Whitlow, J. W., \& Bellwoar, V. (1991). Eating regulation: The role of restraint, dieting and weight. International Journal of Eating Disorders, 10, 461-471.

Loxton, N. J., \& Dawe, S. (2001). Alcohol abuse and dysfunctional eating in adolescent girls: The influence of individual differences in sensitivity to reward and punishment. International Journal of Eating Disorders, 29, 455-462.

Loxton, N. J., \& Dawe, S. (2006). Reward and punishment sensitivity in dysfunctional eating and hazardous drinking women: Associations with family risk. Appetite, 47, 361-371.

Loxton, N. J., \& Dawe, S. (2007). How do dysfunctional eating and hazardous drinking women perform on behavioural measures of reward and punishment sensitivity? Personality and Individual Differences, 42, 1163-1172.

Marsh, D. M., Dougherty, D. M., Mathias, C. W., Moeller, F. G., \& Hicks, L. R. (2002). Comparisons of women with high and low trait impulsivity using behavioral models of response-inhibition and reward-choice. Personality and Individual Differences, 33, 1291-1310.

Mathews, A., \& MacLeod, C. (2002). Induced processing biases have causal effects on anxiety. Cognition and Emotion, 16, 331 - 354. 
Matthys, W., van Goozen, S. H. M., de Vries, H., Cohen-Kettenis, P. T., \& van Engeland, H. (1998). The dominance of behavioural activation over behavioural inhibition in conduct disordered boys with our without Attention Deficit Hyperactivity Disorder. Journal of Child Psychology and Psychiatry, 39, 643-651.

McNair, P. M., Lorr, M. \& Droppleman, L. F. (1992). POMS Manual (Revised 1992). San Diego: Educational and Industrial Testing Service.

McCrae, R. R., \& Costa, P. T. Jr (1990). Personality in adultbood. New York: Guilford. McCrory, M. A., Fuss, P. A., McCallum, J. E., Yao, M., Vinken, A. G., Hays, N. P., et al. (1999). Dietary variety within food groups: association with energy intake and body fatness in men and women. American Journal of Clinical Nutrition, 69, 440447.

Metcalfe, J., \& Mischel, W. (1999). A hot/cool-system analysis of Delay of Gratification: Dynamics of willpower. Psychological Review, 106, 3-19.

Moeller, F. G., Barratt, E. S., Dougherty, D. M., Schmitz, J. M., \& Swann, A. C. (2001). Psychiatric aspects of impulsivity. American Journal of Psychiatry, 158, 1783-1793.

Moeller, F. G. \& Dougherty, D. M. (2002). Impulsivity and substance abuse: What is the connection? Addictive Disorders and Their Treatment, 1, 3-10.

Muraven, M., Tice, D. M. \& Baumeister, R. F. (1998). Self-control as limited resource: Regulatory depletion patterns. Journal of Personality and Social Psychology, 74, 774789.

Nasser, J. A., Gluck, M. E., \& Geliebter, A. (2004). Impulsivity and test meal intake in obese binge eating women. Appetite, 43, 303-307.

Nederkoorn, C., Braet, C., Van Eijs, Y., Tanghe, A., \& Jansen, A. (2006a). Why obese children cannot resist food: The role of impulsivity. Eating Bebaviors, 7 , 315-322.

Nederkoorn, C., Jansen, E., Mulkens, S., \& Jansen, A. (2007) Impulsivity predicts treatment outcome in obese children. Bebaviour Research and Therapy, 45, 1071-1075.

Nederkoorn, C., Smulders, F. T. Y., Havermans, R. C., Roefs, A., \& Jansen, A.(2006b). Impulsivity in obese women. Appetite, 47, 253-256.

Nederkoorn, C., Van Eijs, Y., \& Jansen, A. (2004). Restrained eaters act on impulse. Personality and Individual Differences, 37, 1651-1658.

Nielsen, S. J., \& Popkin, B. M. (2003). Patterns and trends in food portion sizes, 1977-1998. Journal of the American Medical Association, 289, 450-453. 
Nielsen, S. J., \& Popkin, B. M. (2004). Changes in beverage intake between 1977 and 2001. American Journal of Preventive Medicine, 27, 205-210.

Orford, J. (2001). Addiction as excessive appetite. Addiction, 96, 15-31.

Overduin, J. \& Jansen, A. (1997). Conditioned insulin and blood sugar responses in humans in relation to binge eating. Physiology and Bebavior, 61, 569-575.

Painter, J. E., Wansink, B., \& Hieggelke, J. B. (2002). How visibility and convenience influence candy consumption. Appetite, 38, 237-238.

Patton, J. H., Stanford, M. S., \& Barratt, E. S. (1995). Factor structure of the Barratt Impulsiveness Scale. Journal of Clinical Psychology, 51, 768-774.

Powell, J. H., Al-Adawi, S., Morgan, J., \& Greenwood, R. J. (1996). Motivational deficits after brain injury: effects of bromocriptine in 11 patients. Journal of Neurology, Neurosurgery, and Psychiatry, 60, 416-421.

Raynor, H. A., Jeffery, R. W., Phelan, S., Hill, J. O., \& Wing, R. R. (2005). Amount of food group variety consumed in the diet and long-term weight loss maintenance. Obesity Research, 13, 883-890.

Reynolds, B. (2006). A review of delay-discounting research with humans: relations to drug use and gambling. Bebavioural Pharmacology, 17, 651-667.

Reynolds, B., \& Schiffbauer, R. (2005). Delay of gratification and delay discounting: A unifying feedback model of delay-related impulsive behavior. The Psychological Record, $55,439-460$.

Riggs, N. R., Kobayakawa Sakuma, K., \& Pentz, M. A. (2007). Preventing risk for obesity by promoting self-regulation and decision-making skills. Evaluation Review, 31, 287-310.

Roefs, A. \& Jansen, A. (2004). The effects of information about fat content on food consumption in overweight/obese and lean people. Appetite, 43, 319-322.

Rogers, P. J., \& Hill, A. J. (1989). Breakdown of dietary restraint following mere exposure to food stimuli: Interrelationships between restraint, hunger, salivation, and food intake. Addictive Bebaviors, 14, 387-397.

Rolls, B. J., Roe, L. S., Kral, T. V. E., Meengs, J. S., \& Wall, D. E. (2004). Increasing the portion size of a packaged snack increases energy intake in men and women. Appetite, 42, 63-69.

Rolls, E. T., Rolls, B. J., \& Rowe, E. A. (1983). Sensory-specific and motivation-specific satiety for the sight and taste of food and water in man. Physiology and Bebavior, 30, 185-192. 
Rolls, B. J., Rowe, E. A., \& Rolls, E. T. (1982). How sensory properties of foods affect human feeding behavior. Physiology and Bebavior, 29, 409-417.

Rolls, B. J., Rowe, E. A., Rolls, E. T., Kingston, B., Megson, A. \& Gunary, R. (1980). Variety in a meal enhances food intake in man. Physiology \& Bebavior, 30, 185-192.

Rosval, L., Steiger, H., Bruce, K., Israël, M., Richardson, J., \& Aubut, M. (2006). Impulsivity in women with eating disorders: Problem of response inhibition, planning, or attention? International Journal of Eating Disorders, 39, 590-593.

Rotenberg, K. I., Lancaster, C., Marsden, J., Pryce, S., Williams, J., \& Lattimore, P. (2005). Effects of priming thoughts about control on anxiety and food intake as moderated by dietary restraint. Appetite, 44, 235-241.

Rydén, A., Sullivan, M., Torgerson, J. S., Karlsson, J., Lindroos, A.-K., \& Taft, C. (2003). Severe obesity and personality: A comparative controlled study of personality traits. International Journal of Obesity, 27, 1534-1540.

Saelens, B. E., \& Epstein, L. H. (1996). Reinforcing value of food in obese and non-obese women. Appetite, 27, 41-50.

Schacham, S. (1983). A shortened version of the Profile of Mood States. Journal of Personality Assessment, 47, 305-306.

Schoenmakers, T., Wiers, R. W., Jones, B. T., Bruce, G., \& Jansen, A. T. M. (2006). Attentional re-training decreases attentional bias in heavy drinkers without generalization. Addiction, 102, 399-405.

Serdula, M. K., Ivery, D., Coates, R. J., Freedman, D. S., Williamson, D. F., \& Byers, T. (1993). Do obese children become obese adults? A review of the literature. Preventive medicine, 22, 167-177.

Siegel, R. A. (1978). Probability of punishment and suppression of behavior in psychopathic and nonpsychopathic offenders. Journal of Abnormal Psychology, 87, 514-522.

Solanto, M. V., Abikoff, H., Sonuga-Barke, E., Schachar, R., Logan, G. D., Wigal, et al. (2001). The ecological validity of delay aversion and response inhibition as measures of impulsivity in AD/HD: A supplement to the NIMH multimodal treatment study of AD/HD. Journal of Abnormal Child Psychology, 29, 21 5-228.

Sørensen, L. B., Møller, P., Flint, A., Martens, M. \& Raben, A. (2003). Effect of sensory perception of foods on appetite and food intake: a review of studies on humans. International Journal of Obesity, 27, 1152-1166. 
Srull, T. K., \& Wyer, R. S. (1979). The role of category accessibility in the interpretation of information about persons: Some determinants and implications. Journal of Personality and Social Psychology, 37, 1660-1672.

Stice, E., Ozer, S., \& Kees, M. (1997). Relation of dietary restraint to bulimic symptomatology: The effects of criterion confounding of the Restraint Scale. Bebaviour Research and Therapy. 35, 145-152.

Stunkard, A. J., \& Sobal, J. (1995). Psychosocial consequences of obesity. In K. D. Brownell \& C. G. Fairburn (Eds.), Eating disorders and obesity: A comprebensive bandbook (pp. 417-421). New York: Guilford Press.

Tice, D. M., Baumeister, R. F., Shmueli, D., \& Muraven, M. (2007). Restoring the self: Positive affect helps improve self-regulation following ego-depletion. Journal of Experimental Social Psychology, 43, 379-384.

Tiggeman, M., Hargreaves, D., Polivy, J., \& McFarlane, T. (2004). A word-stem completion task to assess implicit processing of appearance-related information. Journal of Psychosomatic Research, 57, 73-78.

Torrubia, R., Ávila, C., Moltó, J., \& Caseras, X. (2001). The Sensitivity to Punishment and Sensitivity to Reward Questionnaire (SPSRQ) as a measure of Gray's anxiety and impulsivity dimensions. Personality and Individual Differences, 31, 837-862.

Van den Hurk, K., van Dommelen, P., van Buuren, S., Verkerk, P. H., \& HiraSing, R. A. (2007, June 29). Prevalence of overweight and obesity in the Netherlands in 2003, compared to 1980 and 1997. Archives of Disease in Childbood, article doi:10.1136/adc.2006.115402. Retrieved September 12, 2007, from http://adc.bmj.com/cgi/reprint/92/11/992.pdf

Van der Ploeg, H. M., Defares, P. B., \& Spielberger, C. D. (1980). Handleiding bij de ZelfBeoordelings Vragenlijst: Een Nederlandstalige bewerking van de Spielberger State-Trait Anxiety Inventory [A manual for the Self-Assessment Questionnaire: A Dutch version of the Spielberger State-Trait Anxiety Inventory]. Swets \& Zeitlinger b.v., Lisse.

Visscher, T. L. S., Viet, A. L., Kroesbergen, H. T., \& Seidell, J. C. (2006). Underreporting of BMI in adults and its effects on obesity prevalence estimations in the period 1998 to 2001. Obesity, 14, 2054-2063. 
Wald, F. D. M., \& Mellenbergh, G. J. (1990). De verkorte versie van de Nederlandse vertaling van de Profile of Mood States (POMS) [The short form of the Dutch translation of the Profile of Mood States (POMS)]. Nederlands Tijdschrift voor de Psychologie, 45, 86-90.

Wansink, B., Painter, J. E., \& North, J. (2005). Bottomless bowls: Why visual cues of portion size may influence intake. Obesity Research, 13, 93-100.

Wansink, B., \& Park, S. B. (2001). At the movies: How external cues and perceived taste impact consumption volume. Food Quality and Preference, 12, 69-74.

Werrij, M. Q., Mulkens, S., Hospers, H., \& Jansen, A. (2006). Overweight and obesity: the significance of a depressed mood. Patient Education and Counseling, 62, 126-131.

Whiteside, S. P., \& Lynam, D. R. (2001). The five factor model and impulsivity: using a structural model of personality to understand impulsivity. Personality and Individual Differences, 30, 669-689.

Williams, B. R., Ponesse, J. S., Schachar, R. J., Logan, G. D., \& Tannock, R. (1999). Development of inhibitory control across the life span. Developmental Psychology, 35, 205-213.

Wilson, G. T. (1994). Behavioral treatment of obesity: Thirty years and counting. Advances in Behavior Research and Therapy, 16, 31-75.

Wingrove, J., \& Bond, A. J. (1997). Impulsivity: a state as well as a trait variable. Does mood awareness explain low correlations between trait and behavioural measures of impulsivity. Personality and Individual Differences, 22, 333-339.

Winstanley, C. A., Eagle, D. M., \& Robbins, T. W. (2006). Behavioral models of impulsivity in relation to ADHD: Translation between clinical and preclinical studies. Clinical Psychology Review, 26, 379-395.

Wonderlich, S. A., Connolly, K. M., \& Stice, E. (2004). Impulsivity as a risk factor for eating disorder behavior. Assessment implications with adolescents. International Journal of Eating Disorders, 36, 172-182.

World Health Organisation (2003). Obesity and overweight.

World Health Organisation (2005). The European health report 2005: Public health action for healthier children and populations. Retrieved October 9, 2006, from bttp://www.wbo.int/wbr/2005/en/ 

Dankwoord 
"Er is een dorp voor nodig om een kind op te voeden" [It takes a village to raise a child] zegt een oud Afrikaans spreekwoord. Hetzelfde geldt voor een promovendus aan het begin van zijn of haar promotietraject. Het succesvol afronden dat traject is niet iets dat je alleen doet. Integendeel. Ik wil hier dan ook alle mensen bedanken die op de één of andere manier hebben bijgedragen aan het proefschrift dat voor u ligt.

Sjaan, je was geweldig als dagelijks begeleider. Je deur stond altijd open als ik ergens tegenaan liep. Ik heb enorm veel geleerd van onze "datadagen" en brainstormsessies. Bedankt daarvoor! Het is vreemd dat je straks mijn begeleider niet meer bent. Ik hoop dat we nog lang mogen samenwerken.

Anita, als promotor hielp jij de nodige knopen doorhakken tijdens onze maandelijkse gesprekken. Je leerde me mijn data naar waarde te schatten, en ze eenvoudig op te schrijven met een duidelijke boodschap. Echte tegenvallers met publiceren heb ik daardoor niet gekend. Bedankt!

Christine en Nicolette, mijn lieve kamergenoten. Bedankt voor jullie interesse in de vorderingen, voor het geduld met mijn bureau die alsmaar meer rommel vergaarde, voor de APA raad, en voor de vele vakinhoudelijke, maar ook gezellige gesprekken over verbouwingen, zwanger zijn en zoveel meer.

Ellen en Martien, als kamergenoot heb ik bij jullie beide de promotiestress van dichtbij meegemaakt. Ik was er al helemaal aan gewoon toen het er voor mij aankwam! Ook al zijn we nu geen kamergenoten meer, onze lunches en pizza's bij de "kitsch Italiaan" waren een welkome verademing.

Anita, Carolien, Sjaan, Remco, Sandra, Anne, Jen, Hugo, Esther, Elke, Janneke en Nicolette, ofwel de eetgroep. Het is enorm fijn om zo'n grote groep collega's te hebben om mee te brainstormen en op congres te gaan. Ook de vele gezellige etentjes en borrels heb ik enorm gewaardeerd! Hugo, jou wil ik speciaal bedanken voor het design van de cover en je goeie raad bij het lay-outen. Je bent een prachtcollega! Esther en Elke, ik ben heel blij en fier dat jullie mijn paranimfen willen zijn! 
De andere collega's op de derde verdieping: bedankt voor de gesprekken in de keukenhoek, de gezellige dagjes-uit, en voor het opfleuren van de EPP cursussen die we als promovendi dienden te volgen.

Jessie en Truus, bedankt voor jullie hulp met allerlei praktische zaken, maar vooral voor het feit dat jullie het altijd met een glimlach deden.

Tijdens dit project zijn heel wat data verzameld, en dat heb ik niet allemaal alleen gedaan. Dank aan Kasia Stankiewicz, Nicole Geschwind, Sandra Sassen, Roefje Janssen, Nelleke de Doelder, en Loes Peeters voor hun hulp hierbij.

De meest arbeidsintensieve studie was de "kindjesstudie" (hoofdstuk 5). Daar had ik heel wat kinderen voor nodig die allemaal een uur uit de klas moesten om individueel getest te worden. Mijn welgemeende dank aan de directies, ouders en kinderen van de scholen in Maasmechelen die toch wilden meedoen: Basisschool op het Boseind, Vrije Basisschool Proosterbos, Vrije Basisschool Opgrimbie, Vrije Basisschool Leut, en Vrije Basisschool Sint-Jan.

Marijke en Julie. Tijdens onze studie hier hebben we veel plezier gehad. Daarna gingen jullie je eigen weg en ik bleef als enige hier om te promoveren. Toch hebben we altijd contact gehouden via e-mail en regelmatig ook in levende lijve. Marijke, bedankt voor de gezellige etentjes en lange gesprekken, en Julie voor de zwangerschapsmails en je aanstekelijke sportiviteit.

Bomma, Lucie en mama. Waar moet ik beginnen? Zolang ik me kan herinneren zijn jullie mijn drie musketiers. Altijd daar om me te steunen en te beschermen. Een simpel "Dank u" is bijlange na niet genoeg. Hopelijk kan ik jullie nog veel momenten van plezier en trots bezorgen.

Kris, bedankt voor alles. 

Curriculum Vitae 
Ramona Guerrieri was born in Genk (Belgium) on July 8, 1977. In June 1995 she graduated from secondary school Koninklijk Atheneum in Maasmechelen (Belgium). In September 1995 she started teacher training and graduated June 1998 (magna cum laude) From September 1998 until June 2000 she taught English, Dutch and history in several secondary schools. She started studying psychology at Maastricht University in September 2000 and graduated in June 2004 (cum laude). Immediately following graduation she started her PhD project at the Department of Experimental Psychology at Maastricht University. Since January 2008 she is a postdoctoral fellow at the Department of Clinical Psychological Science (formerly the Department of Experimental Psychology) in Maastricht.

\section{Publications}

Guerrieri, R. (2005). Toen de koekjestrommel de fruitschaal verdrong... De toxische omgeving als oorzaak van obesitas. [The toxic environment as a cause of obesity] De Psycholoog, 40, 642-646.

Guerrieri, R., Nederkoorn, C., Jansen, A. (in press). The effect of an impulsive personality on overeating and obesity: Current state of affairs. Psychological Topics.

Guerrieri, R., Nederkoorn, C., Jansen, A. (2008). The interaction between impulsivity and a varied food environment: its influence on food intake andoverweight. International Journal of Obesity, 32, 708-7 14.

Guerrieri, R., Nederkoorn, C., \& Jansen, A. (2007a). How impulsiveness and variety influence food intake in a sample of healthy women. Appetite, 48, 199-122.

Guerrieri, R., Nederkoorn, C., Stankiewicz, K., Alberts, H., Geschwind, N., Martijn, C., \& Jansen, A. (2007b). The influence of trait and induced state impulsivity on food intake in normal-weight healthy women. Appetite, 49, 66-73.

Claes, L., Nederkoorn, C., Vandereycken, W., Guerrieri, R., \& Vertommen, H. (2006). Impulsiveness and lack of inhibitory control in eating disorders. Eating Bebaviors, 7, 196-203.

Geraerts, E., Jelicic, M., Merckelbach, H. \& Guerrieri, R. (2004). Het effect van acute stress op het neutraal en emotioneel geheugen. [The effect of acute stress on neutral and emotional memory] Neuropraxis, 8, 54-59.

Havermans, R. C., Jansen, E., Guerrieri, R., \& Jansen, A. (2007). Dikke kijkbuiskinderen of waarom kinderen dik worden van televisiekijken. De Psycholoog, 42, 582-587. 
Jelicic, M., Geraerts, E., Merckelbach, H. \& Guerrieri, R. (2004). Acute stress enhances memory for emotional words, but impairs memory for neutral words. International Journal of Neuroscience, 114, 1343-1351.

Nederkoorn, C., Guerrieri, R., \& Jansen, A. (2006). Leven in luilekkerland. [Living in the land of plenty] De Psycholoog, 41, 10-15.

Manuscripts submitted for publication

Guerrieri, R., Nederkoorn, C., Schrooten, M., Martijn, C., \& Jansen, A. (2008). Inducing impulsivity leads restrained eaters into overeating, whereas current dieters stick to their diet.

Jansen, A., Nederkoorn, C., van Baak, L., Keirse, C., Guerrieri, R., \& Havermans, R. (2008). High-restrained eaters only overeat when they are also impulsive.

Nederkoorn, C., Guerrieri, R., Roefs, A., \& Jansen, A. (2008). Why impulsive people should not go to the supermarket when bungry; bunger and response inbibition interact in their effect on consumptive bebaviour.

\section{Conference presentations}

Guerrieri, R., Nederkoorn, C., \& Jansen, A. (2005). How impulsiveness and variety influence food intake in a sample of bealthy women. Talk given at the Endo Neuro Psycho meeting, May 31-June 3 2005, Doorwerth, The Netherlands.

Guerrieri, R., Nederkoorn, C., \& Jansen A. (2005). How impulsiveness and variety influence food intake in a sample of bealthy women. Poster presented at the annual meeting of the International Society of Behavioral Nutrition and Physical Activity (ISBNPA), June 16 - 18, 2005, Amsterdam, the Netherlands.

Guerrieri, R., Nederkoorn, C., \& Jansen A. (2005). Do impulse control problems cause overweight / obesity? Poster presented at the annual meeting of the Eating Disorders Research Society (EDRS), September 29 - October 1, 2005, Toronto, Canada.

Guerrieri, R., Nederkoorn, C., \& Jansen, A. (2006). Do impulse control problems cause obesity? Talk given at the British Feeding and Drinking Group Annual Meeting, April 20-21, 2006, Birmingham, United Kingdom.

Guerrieri, R., Nederkoorn, C., \& Jansen, A. (2006). The effect of variety and impulsivity on food intake in primary school children. Poster presented at the International Conference on Eating Disorders (ICED), June 7-10, 2006, Barcelona, Spain. 
Guerrieri, R., Nederkoorn, C., \& Jansen, A. (2007). Impulsivity, variety and the risk for overweight and obesity in primary school children. Talk given at the Experimental Psychopathology Symposium "The development of addictive behaviours and eating disorders", May 10-11, Heeze, the Netherlands.

Guerrieri, R., Nederkoorn, C., Schrooten, M., Martijn, C., \& Jansen, A. (2007). Inducing impulsivity versus control/inbibition affects food intake in bealtby women. Talk given at the British Feeding and Drinking Group Annual Meeting, April 2-3, 2007, Newcastle, United Kingdom.

Guerrieri, R., Nederkoorn, C., Schrooten, M., Martijn, C., \& Jansen, A. (2007). The interaction between impulsivity and a varied food environment: Its link to food intake and overweight. Talk given at the World Congress of Behavioural and Cognitive Therapies, July 11-14, 2007, Barcelona, Spain.

\section{Awards}

2005: Award for young authors, presented by "De Psycholoog" [The Psychologist], a Dutch psychological journal.

2007: Nomination for the article prize, presented by Experimental Psychopathology (EPP), the Dutch-Flemish postgraduate school for research and education 